San Jose State University

SJSU ScholarWorks

Master's Theses

Master's Theses and Graduate Research

1998

\title{
Synthesis and characherizations [sic] of endcapped C-18 stationary phases for HPLC
}

Raymond Jin Yu

San Jose State University

Follow this and additional works at: https://scholarworks.sjsu.edu/etd_theses

\section{Recommended Citation}

Yu, Raymond Jin, "Synthesis and characherizations [sic] of endcapped C-18 stationary phases for HPLC" (1998). Master's Theses. 1786.

DOI: https://doi.org/10.31979/etd.5vf5-9zex

https://scholarworks.sjsu.edu/etd_theses/1786

This Thesis is brought to you for free and open access by the Master's Theses and Graduate Research at SJSU ScholarWorks. It has been accepted for inclusion in Master's Theses by an authorized administrator of SJSU ScholarWorks. For more information, please contact scholarworks@sjsu.edu. 


\section{INFORMATION TO USERS}

This manuscript has been reproduced from the microfilm master. UMI films the text directly from the original or copy submitted. Thus, some thesis and dissertation copies are in typewriter face, while others may be from any type of computer printer.

The quality of this reproduction is dependent upon the quality of the copy submitted. Broken or indistinct print, colored or poor quality illustrations and photographs, print bleedthrough, substandard margins, and improper alignment can adversely affect reproduction.

In the unlikely event that the author did not send UMI a complete manuscript and there are missing pages, these will be noted. Also, if unauthorized copyright material had to be removed, a note will indicate the deletion.

Oversize materials (e.g., maps, drawings, charts) are reproduced by sectioning the original, beginning at the upper left-hand comer and continuing from left to right in equal sections with small overlaps. Each original is also photographed in one exposure and is included in reduced form at the back of the book.

Photographs included in the original manuscript have been reproduced xerographically in this copy. Higher quality 6" x 9" black and white photographic prints are available for any photographs or illustrations appearing in this copy for an additional charge. Contact UMI directly to order.

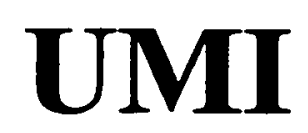

A Bell \& Howell Information Company 



\section{NOTE TO USERS}

The original manuscript received by UMI contains pages with slanted, light, and/or indistinct print. Pages were microfilmed as received.

This reproduction is the best copy available 



\title{
SYNTHESIS AND CHARACHERIZATIONS OF ENDCAPPED C-18 STATIONARY PHASES FOR HPLC
}

\author{
A Thesis \\ Presented to \\ The Faculty of the Department of Chemistry \\ San Jose State University \\ In Partial Fulfillment \\ of the Requirements for the Degree \\ Master of Science
}

by

Raymond Jin Yu

December 1998 
UMI Number: 1392851

UMI Microform 1392851

Copyright 1999, by UMI Company. All rights reserved.

This microform edition is protected against unauthorized copying under Title 17, United States Code.

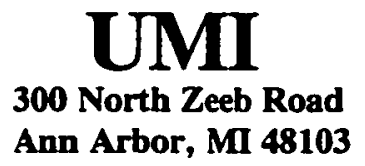


(C) 1998

Raymond Jin Yu

ALL RIGHTS RESERVED 
APPROVED FOR THE DEPARTMENT OF CHEMISTRY

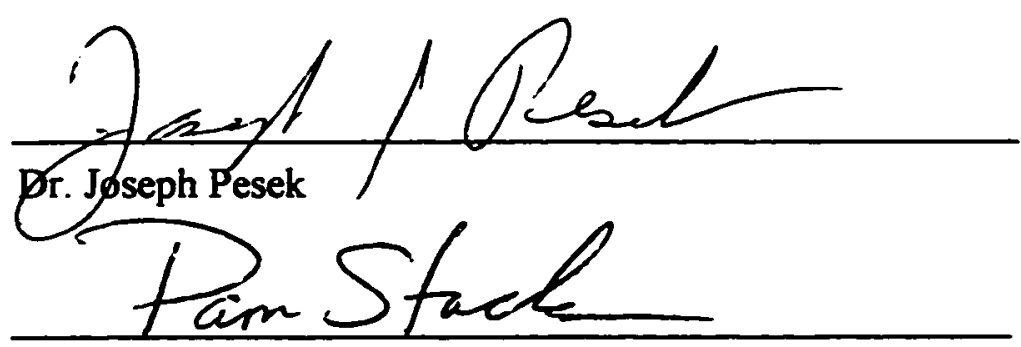

Dr. Pam Stacks

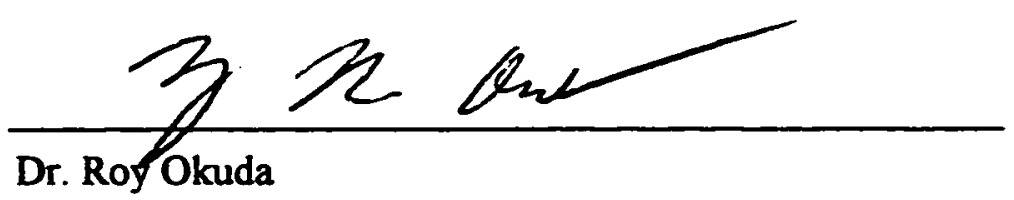

APPROVED FOR THE UNIVERSITY

William Disk 


\begin{abstract}
ABSTRCT
SYNTHESIS AND CHARACTERIZATIONS OF ENDCAPPED STATIONARY PHASE FOR HPLC

by Raymond $\mathrm{Yu}$
\end{abstract}

Endcapping is a secondary silanization reaction intended to derivatize residual silanols on a silica surface. The popular endcapping method is to use trimethylchlorosilane (TMCS) and hexamethyldisilazane (HMDS) as endcapping reagents. The primary modification is triethoxysilane (TES) silanization followed by C-18 hydrosilation.

Two HPLC stationary phases were synthesized; they were C-18 bonding before endcapping (BBE) and endcapping before C-18 bonding (EBB). Spectroscopic means including diffuse reflectance infrared Fourier transform spectroscopy (DRIFT) and nuclear magnetic resonance spectroscopy (NMR) were used to qualitatively characterize the modified surfaces, and elemental analysis was performed to quantitatively characterize the surfaces. Chromatographic characterizations on both columns were performed using different types of probes. Chromatographic temperature effect and $\mathrm{pH}$ stability were also studied. 


\section{AKNOWLEGEMENT}

First, I would like to give my sincere thanks to my research advisor, Dr. Joseph Pesek, and his research scientist, Maria Matyska-Pesek, for giving me not only an excellent research project, but also tremendous helps and guidance throughout my research study.

I would like to thank my committee members, Dr. Pam Stacks and Dr. Roy Okuda, for reviewing my thesis and giving valuable comments. An acknowledgment is also made to Jerry Higgins from Higgins Analytical Inc. for packing three HPLC columns for me.

Last, and certainly not the least, I want to thank my parents, my sister, my brother-in-law, my nieces and many others for standing by me. It would be impossible for me to complete my graduate study without their supports both financially and emotionally. 
"It is the great beauty of our science that advancement in it, whether a degree great or small, instead of exhausting the subject of research, opens the doors to further and more abundant knowledge, overflowing with beauty and utility." 


\section{TABLE OF CONTENTS}

\section{CHAPTER I - INTRODUCTION}

A. High Performance Liquid Chromatography 1

B. Silica Surface and Modification

1. Silica and Its Modification Methods

2. Monomeric Octadecyl Silica Bonded Phase

C. Endcapping

D. Endcapped Monomeric Octadecyl Silica Bonded Phases

E. Characterization Techniques

1. Spectroscopic Techniques

a. Diffuse Reflectance Fourier Transform Infrared Spectroscopy (DRIFT)

b. Nuclear Magnetic Resonance (NMR) Spectroscopy

2. Elemental Analytical Methods

F. Aims of The Work

\section{CHAPTER II - EXPERIMENTAL}

A. Materials

1. Chemicals

2. Chemicals for Synthesis of Endcapped Stationary Phases

B. Experimental

1. Preparation of Speier's Catalyst

2. Synthesis of Silica Hydride via Silanization

3. Synthesis of Bonding Before Endcapping Stationary Phase

4. Synthesis of Endcapping Before Bonding Stationary Phase

5. Column Packing

C. Characterization Methods

1. Diffuse Reflectance Fourier Transform Infrared Spectroscopy (DRIFT)

2. Cross-polarization, Magic Angle Spinning NMR (CP-MAS NMR) 
3. Elemental Analysis 22

4. High Performance Liquid Chromatography 23

\section{CHAPTER II - RESULTS AND DISCUSSION}

A. Elemental Analysis Results 25

B. Spectroscopic Characterization of Silica Hydride 25

C. Spectroscopic Characterizaton of BBE Bonded Phase

1. Spectroscopic Characterizations Vydac Hydride Bonded to C-18 28

2. Spectroscopic Characterizations Vydac C-18 Endcapped With 32 TMCS/HMDS

D. Spectroscopic Characterization of EBB Bonded Phases

1. Spectroscopic Characterization of Vydac Hydride Endeapped 36 With TMCS/HMDS

2. Spectroscopic Characterization of Endcapped Vydac Bonded to 36 C-18

E. Chromatographic Evaluation 40

1. Isocratic Separation of Perkin Elmer Universal Test Mix 44

2. Isocratic Separation of Standard Reference Mirture (SRM 869) 45

3. Polycyclicaromatic Hydrocarbons Compounds 49

4. Basic Organic Compounds 53

F. Chromatographic Study on Temperature Effect 56

G. Chromatographic Studies on pH Effects

1. High pH Stability Study 56

2. Low pH Stability Study 76

$\begin{array}{lr}\text { CHAPTER IV - CONCLUSION } & 79\end{array}$

CHAPTER V - REFERENCES 


\section{LIST OF FIGURES}

Figure 1 Active groups present on a silica surfaces
a. siloxane
b. "free" silanol
c. geminol
d. vicinol ("bound" silanol)
e. bound water

Figure 2 Methods for modification of a silica surface.

Figure 3 Endcapping reaction scheme by using TMCS and HMDS (1:1 11 molar ratio).

Figure 4 Mechanisms for synthesis of endcapped C18 stationary phases. 13

Figure 5 The DRIFT spectrum of Vydac silica hydride. 26

Figure 6 The CP-MAS ${ }^{29}$ Si NMR spectrum of Vydac silica hydride. 27

Figure $7 \quad$ CP-MAS ${ }^{13} \mathrm{C}$ NMR spectrum of Vydac silica hydride. 29

Figure 8 The DRIFT spectrum of C18 Bonded to Vydac hydride. 30

Figure 9 The CP-MAS ${ }^{13} \mathrm{C}$ NMR spectrum of $\mathrm{C} 18$ Bonded to Vydac 31 hydride.

Figure 10 The DRIFT spectrum of Vydac C-18 endcapped with TMCS/HMDS.

Figure 11 The CP-MAS ${ }^{29}$ Si NMR spectrum of Vydac C-18 endacapped with TMCS/HDMS.

Figure 12 The CP-MAS ${ }^{13} \mathrm{C}$ NMR spectrum of Vydac C-18 endcapped with TMCS/HMDS.

Figure 13 The DRIFT of Vydac hydride endcapped with TMCS/HMDS.

Figure $14 \quad$ CP-MAS ${ }^{29} \mathrm{Si}$ NMR spectrum of Vydac hydride endcapped with TMCS/HMDS.

Figure 15 The CP-MAS ${ }^{13} \mathrm{C}$ NMR of Vydac hydride endcapped with 
TMCS/HMDS (1:1 molar ratio).

Figure 16 The DRIFT of C18 Bonded to endcapped Vydac hydride 41

Figure $17 \quad \mathrm{CP}_{\mathrm{MAS}}{ }^{29} \mathrm{Si}$ NMR of C18 Bonded to endcapped Vydac $\quad 42$ Hydride.

Figure 18 The CP-MAS ${ }^{13} \mathrm{C}$ NMR of $\mathrm{C} 18$ Bonded to endcapped Vydac hydride.

Figure 19 Reversed phase separation of PE universal test mixture on 46 BBE.

Figure 20 Reversed phase separation of PE universal test mixture on EBB.

Figure 21 Reversed phase separation of PE universal test mixture on Pheno C18.

Figure 22 Reversed phase separation of NIST mix (SRM 869) on BBE. 50

Figure 23 Reversed phase separation of NIST mix (SRM 869) on EBB 51

Figure 24 Reversed phase separation of NIST mix (SRM 869) on Pheno 52 C18.

Figure 25 (a) Chromatographic studies of PAH compounds on BBE. 54

Figure 25 (b) Chromatographic studies of PAH compounds on EBB. 55

Figure 26 (a) Chromatographic studies of p-toluidine on peak symmetry. 57

Figure 26 (b) Chromatographic studies of pyridine on peak symmetry. 58

Figure 27 (a) Chromatogram of pyridine on BBE. 59

Figure 27 (b) Chromatogram of pyridine on EBB 60

Figure 27(c) Chromatogram of pyridine on Pheno C18. 61

Figure 28 (a) Chromatographic study of temperature effect of retention on 62 BBE. 
Figure 28 (b) Chromatographic study of temperature effect of retention on 63 EBB

Figure 29 (a) Chromatographic study of temperature effect on peak shape. 64 Solute: aniline.

Figure 29 (b) Chromatographic study of temperature effect on peak shape. 65 Solute: $\mathrm{n}$, -dimethylaniline.

Figure 29(c) Chromatographic study of temperature effect on peak shape. 66 Solute: diphenylamine.

Figure $30 \quad$ High pH stability chromatographic study on retention. Analyte: 68 pyrene.

Figure 31 High pH stability chromatographic study on \% change in k'. Analyte: pyrene.

Figure 32 High pH stability chromatographic study on retention. Analyte: pyrene.

Figure 33 High pH stability chromatographic study on \% change in $\mathbf{k}^{\prime}$. Analyte: pyrene.

Figure 34 High pH chromatographic stability study on peak shape. Analyte: pyrene.

Figure 35 High pH chromatographic stability study on \% change in asymmetry factor. Analyte: pyrene

Figure $36 \quad$ High pH chromatographic stability study on theoretical plates. 74 Column: BBE. Analyte: pyrene.

Figure $37 \quad$ High pH chromatographic stability study on \% change in theoretical plate. Column: BBE. Analyte: pyrene

Figure 38 Low pH stability study on BBE. Analyte: pyrene

Figure 39 Low pH stability study of \% change in k' on BBE. Analyte: 78 pyrene. 


\section{LIST OF TABLES}

Table 1 Base Packing Materials for HPLC. 3

Table 2 The CAS Registry Numbers of Chemicals Used In this Project. 17

Table 3 Percent Carbon and Surface Coverage Data Table. 25

Table 4 Resolution $\left(R_{s}\right)$ for Perkin Elmer Universal Test Mix (PE-UTM) 45 Separated by Three Columns.

Table 5 Resolution ( $R_{s}$ ) for Standard Reference Mixture (SRM 869) Separated by Three Columns. 


\section{CHAPTER I}

\section{INTRODUCTION}

\section{A. High Performance Liquid Chromatography}

High Performance Liquid Chromatography (HPLC) is one of the most powerful analytical separation techniques in chemical, biochemical and related fields. HPLC is a separation technique which originated from liquid chromatography (LC). Unlike gas chromatography (GC), LC can handle molecules that are thermally labile, polar and or high molecular weight. The most important components of a basic LC system are a solid stationary phase and a liquid mobile phase. Two major mechanisms have been proposed for separation of a mixture by LC; they are adsorption and partitioning. When a mixture is introduced onto the stationary phase, and washed through the stationary phase by the mobile phase, if one component of that mixture is absorbed weakly on the surface of the stationary phase, it will travel faster than the one more strongly absorbed. Hence, separation of these two components occurs. This mechanism is called adsorption. Another proposed mechanism by which separation occurs in the difference in partition coefficients of solutes between the stationary phase and the mobile phase. Such a mechanism is called partition chromatography.'

In the late 1960s, HPLC gradually arose with the introduction of packing materials with smaller particle sizes. Such micro particles have diameters ranging from 3 to $10 \mu \mathrm{m}$. These smaller particles lead to a considerable resistance to the flow so that the mobile phase has to be pumped through the column. These columns are typically 10 to $25 \mathrm{~cm}$ 
long and $4.6 \mathrm{~mm}$ in internal diameter. The introduction of HPLC has greatly improved the efficiency of LC. ${ }^{1,2}$ There are basically two modes in HPLC; they are called normal phase and reverse phase. Normal phase means the polarity of the stationary phase is higher than that of the mobile phase, whereas reverse phase means the polarity of the stationary phase is less than that of the mobile phase. So far, reverse phase is the most popular mode because of its wide range of applications. In reverse phase chromatography, more polar components of a mixture elute faster than less polar ones.

In HPLC, there are several parameters used for retention measurement and column performance. It is known that solutes are separated based on differences in their retention on the column. Peaks are identified by retention times. However, because of the differences in column length and mobile phase flow rate, a capacity factor ( $\left.k^{\prime}\right)$ is used to locate peaks. Capacity factor $\left(k^{\prime}\right)$ is given by $\left(t_{R}-t_{0}\right)$ divided by $t_{0}$, where $t_{R}$ is the retention of a retained solute peak and to is the retention of unretained reference peak. Another parameter, selectivity or separation factor $(\alpha)$, is used to define the separation between two peaks whose value is not less than $1 . \alpha$ is defined by the following equation:

$$
\alpha=k_{2}^{\prime} / k_{i}^{\prime}=\left(t_{R 2}-t_{0}\right) /\left(t_{R 1}-t_{0}\right)
$$

The degree of separation of one component from another is described by the resolution (Rs), and it is measured by the following equation:

$$
R s=\left(t_{R 2}-t_{R 1}\right) /(0.5(w 1+w 2))
$$

where $t_{R}$ is the retention of components 1 and 2 , and $w$ is width of the peaks. When $R s$ is equal to 1.5, two adjacent peaks are just resolved at base line. In terms of column 
performance, one problem is the peak dispersion as the band of solute is moving through the column; longer the solute spends in the system, the more disperse the band becomes. Obviously, smaller peak widths indicate that the column is more efficient. To measure efficiency, plate number $(\mathrm{N})$ and plate height $(\mathrm{H})$ are empirically defined as follows:

$$
N=5.54\left(t_{R} / w_{12}\right)^{2}
$$

or

$$
\mathrm{H}=\mathrm{L} / \mathrm{N}
$$

where $L$ is the column length.

The another important parameter is the asymmetry factor $\left(A_{6}\right)$ which measures the tailing of a peak. $\mathrm{A}_{\mathrm{a}}$ is defined as the right half of the peak width at $10 \%$ of its peak height divided by the left half. When a peak is perfectly symmetrical, $A_{8}$ will equal 1 . Higher values of $A_{4}$ mean greater peak tailing.

\section{B. Silica Surface and Modification}

\section{Silica and Its Modification Methods}

In order to achieve better and faster separations, chromatographers have tried to find different base packing materials for the HPLC stationary phase. Table 1 shows commonly used base materials for HPLC columns.

\begin{tabular}{|c|c|c|}
\hline Base Material & Samples & Usage (\%) \\
\hline Silica Gel & High surface area, wide pore & 75 \\
\hline Polymer & $\begin{array}{l}\text { Polymethacrylates, PS-DVB } \\
\text { polyehthylene glycol }\end{array}$ & 20 \\
\hline Alumina and others & Neutral, acidic Graphitized carbon, zirconia & 5 \\
\hline
\end{tabular}

\section{Table 1: Base Packing Materials for HPLC. ${ }^{3}$}


Among the different base materials shown in Table 1 , it is not difficult to see that so far silica gel is still the most commonly used one for HPLC. Silica has several advantages. First of all, silica gel is rigid and has a high surface area. In addition, it is available with different particle sizes and shapes. Last but not the least, silica gel can be used on both aqueous and non-aqueous environments. ${ }^{1.23}$ It is commonly believed that there are several active sites on the silica surface. They are shown in Figure $1^{2}$. The first group is the siloxane in which one oxygen atom is bonded to two silicon atoms. Because this oxygen is locked in space, it will not have much interaction with solute molecules. Therefore, siloxane is normally considered neutral. The second active group is called a "free" silanol. Because a "free" silanol is highly polar, it can have strong interactions with polar solute molecules. A "free" silanol is considered to be highly acidic, so it strongly absorbs basic molecules. Sometimes this interaction can be an irreversible process. The next active functional group present on the silica surface is what is called a geminol group. Geminols have two - $\mathrm{OH}$ groups attached on the same silicon atom. Because these two $\mathrm{OH}$ groups are so close to each other, they are much more constrained compared to the $\mathrm{OH}$ on a "free" silanol in terms of chance of interactions with solutes. The next active group on the surface is what is called vicinol or "bound' water, that is a hydrogen of one silanol forms a hydrogen bond with an oxygen of an adjacent silanol. The last active group on the surface is what is called bound water, that is a water molecule on the surface forming hydrogen bonding with hydrogens of two neighbored silanols. In general, a "free" silanol is considered to be the one responsible for most of the absorption with polar 


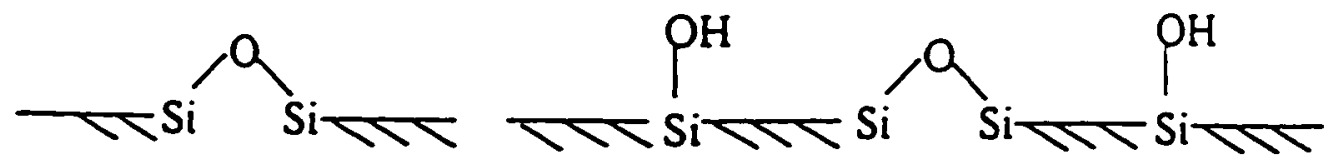

b
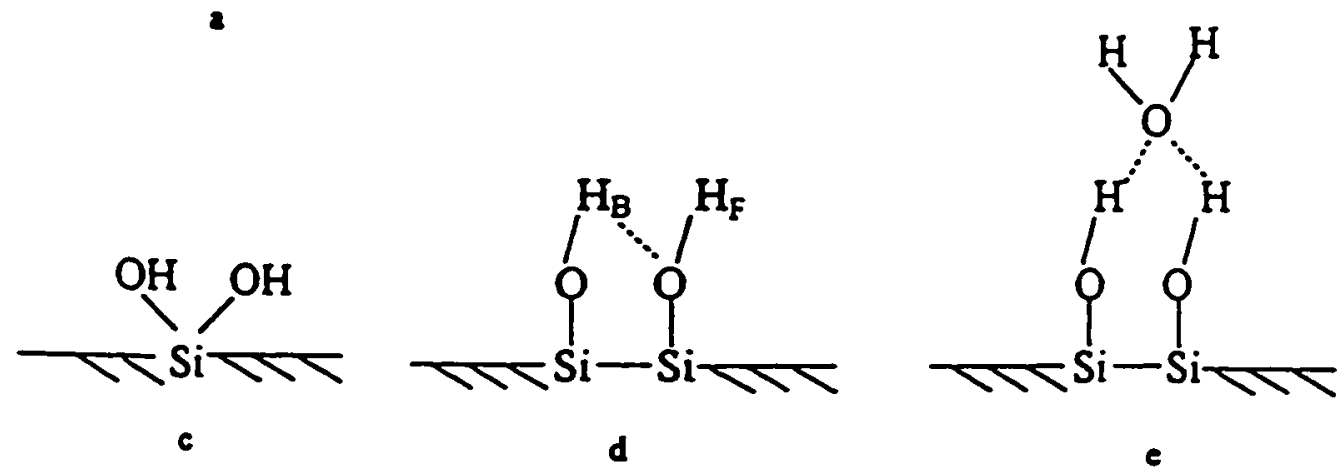

Figure 1. Active groups present on a silica surface: a) silozane, b) "(iree" silanol, c) geminol, d) vicinol ("bound" silnnol), e) bound water. 
solutes. Especially it is considered to be the "bad" silanol when it becomes to the interaction with basic compounds. ${ }^{1,23}$

Throughout the history of reverse phase chromatography, chromatographers have tried different synthetic methods to attach organic moieties onto the silica surface based on the chemistry of silanol. The purpose of these developments is to decrease the polarity of the surface. Some of the commonly used methods are summarized in Figure $2^{4}$. The first, and the most primitive one, is esterification. The chemistry is the reaction between a alcohol with the silanols to form Si-O-C linkages. However, the linkages were found to be very unstable in aqueous environments. This method was soon replaced by organosilanization. The chemistry involves an organosilane regent to react with the silanols. Two approaches have been taken. First, one may use an organosilane reagent with a single reactive group such as chlorine, methoxy or ethoxy to react with silanols. As a result, a single point of attachment of the organic moiety onto the silanol is formed. This single layer is called a monomeric stationary phase. The second approach is to use an organosilane with three reactive groups. The reaction would give a cross-linked multilayer between the neighboring organic moieties. The multi-layer is called a polymeric stationary phase. The linkages by organosilanization are stable in water compared to esters. There is one drawback of this method; an acid is formed during the reaction. ${ }^{4}$ It is well known that the siloxane linkage is fragile in acidic conditions. Si-O-Si-C might be potentially hydrolyzed by the acid formed during the organosilanization reaction.

The third approach involves chlorination of silanols with thionyl chloride followed by the attachment of the organic moiety onto the surface via a Grignard reagent or 
organolithium compound. The bonded phase is more stable hydrolytically compared to that formed by organosilanization. The major drawback of this method is that extremely dry conditions have to be maintained during the synthesis.

One recently developed method ${ }^{5}$ is to use triethoxysilane (TES) followed by hydrosilation. The first step involves a reaction between silanols and TES to form a densely packed hydride monolayer on the surface. In the second step, under proper catalysis, these hydrides react with terminal olefins and a Si-C monomeric layer is formed. This method is advantageous compared to conventional organosilanization in that the linkages are more stable since acid is not formed by this reaction scheme. The hydride monolayer is considered be to less "acidic" than a layer formed through an organosilanization reaction. ${ }^{4,5}$

\section{Monomeric Octadecyl Silica Bonded Phase}

Many organic moieties have been bonded to silica surfaces. However, in reverse phase chromatography, an octadecyl silica (ODS) bonded phase is the most commonly used one because of its wide range of applications. The major separation mechanism for ODS is hydrophobic interaction between the stationary phase and solutes. It has been proposed that the ODS bonded phase can form pockets on the silica surface. ${ }^{6}$ Solutes can diffuse into these pockets, and partitioning occurs between the more hydrophobic side of the pocket and more hydrophilic outside of the pocket. The drawback for the ODS bonded phase is that basic solutes strongly interact with residual silanols on the surface. Such interaction can cause severe tailing. To overcome such limitations, one can either use acidic mobile phases $(\mathrm{pH}<4)$ to protonate basic solutes or basic mobile phases to 


\begin{tabular}{|c|c|c|}
\hline REAETION TYPE & REAETION & $\begin{array}{l}\text { SURFAEE } \\
\text { LIMKAEES }\end{array}$ \\
\hline ESTERIFICA:IOA & S. OOM.R.OH S. OOR M:O & 5. 06 \\
\hline $\begin{array}{l}\text { ORGANO- } \\
\text { SIGAMIZTION }\end{array}$ & 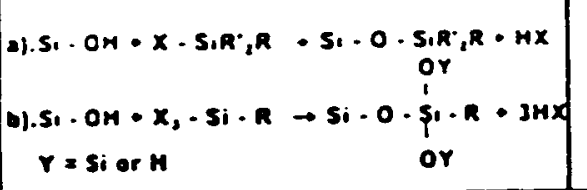 & si.o.si.c \\
\hline 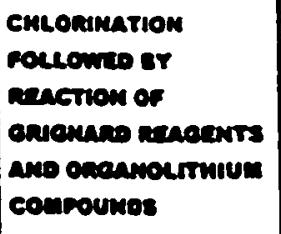 & 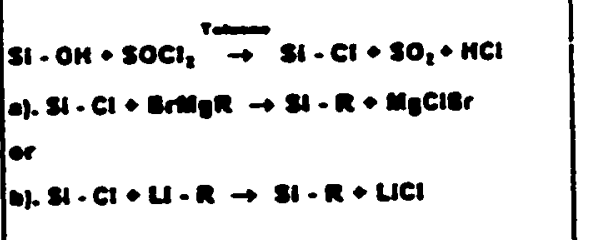 & u.c \\
\hline $\begin{array}{l}\text { 0). res } \\
\text { stcamization }\end{array}$ & 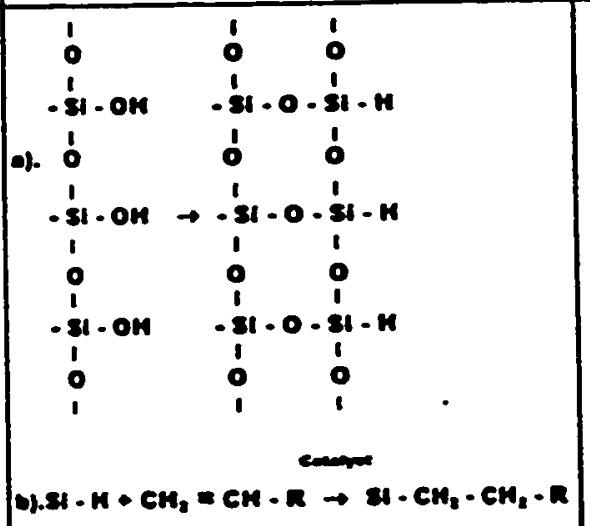 & o. $\mathbf{A . c}$ \\
\hline
\end{tabular}

Figure 2. Methods for modification of a silica surface. 
deprotonate silanols. The purpose is to reduce the interaction between silanols and basic solutes. However, this adjustment may potentially jeopardize the life-time of the stationary phase. It is known that silica bonded phases are $\mathrm{pH}$ sensitive. A column is considered to be most stable between $\mathrm{pH} 2$ to 7.5. Below this range, the bonded phases tend to be removed by hydrolytic cleavage. Above this range, the bonded phase silica tend to dissolve. One common method to remove these "bad" residual silanols is to endcap the surface. Endcapping is normally done by introducing small organosilane molecules.

These organosilanes will either react with some of the silanols, or block access of solutes to some residual silanols. ${ }^{7}$ Endcapping will be discussed in more detail in the next section.

\section{Endcapping}

In spite of different reaction schemes developed to derivatize silanols on a silica surface, still approximately $50 \%$ of silanols remained unmodified. The main reason is due to the steric hindrance effect. To overcome the problem of steric hindrance, endcapping is preferred after the first modification. Endcapping refers to a secondary derivatization reaction intended to shield the residual silanols on a modified surface. Endcapping reagents are small silane reagents that can further react with those silanols which were not accessible to those bulky silanes used during the primary modification process. Since the shape of the silica surface is somewhat irregular, endcapping cannot derivatize all of the residual silanols. However, numerous experiments have proven that endcapping can definitely improve the hydrophobicity of the surface. ${ }^{7,8,9,10}$ 
Two endcapping reagents have been commonly used. They are trimethylchlorosilane (TMCS) and hexamethyldisilazane (HMDS). Their structures are shown below:<smiles>C[Si](C)(C)Cl</smiles>

Trimethylchlorosilane (TMCS)<smiles>C[Si](C)(C)N[Si](C)(C)C</smiles>

Hexamethyldisilazane (HMDS)

There have been three commonly used endcapping reaction schemes proposed ${ }^{10}$. The first approach is to use TMCS as an endcapping reagent to react with residual silanols. This method is not favorable because $\mathrm{HCl}$ is one of the products. As mentioned previously, acid tends to harm the siloxane linkages. A milder approach is to use HMDS as the endcapping reagent. One drawback of this approach is that $\mathrm{NH}_{4} \mathrm{OH}$ is formed during the reaction. It is well known that silica bonded phases tend to be dissolved under basic conditions. The preferred method used by Akapo et. al. ${ }^{14}$ is to use both TMCS and HMDS (1:1 molar ratio) as the endcapping reagents. The reaction scheme is shown in Figure 3. This method is the best compared to the previous two processes in that $\mathrm{NH}_{4} \mathrm{Cl}$ is formed which is not harmful to the bonded phase.

\section{Endcapped Monomeric Octadecyl Silica Bonded Phases}

As described in previous sections, ODS is the most popular stationary phase in reverse phase HPLC. Endcapping can improve column efficiency by derivatization of the residual silanols. To synthesize an endcapped ODS stationary phase, one can use the most 

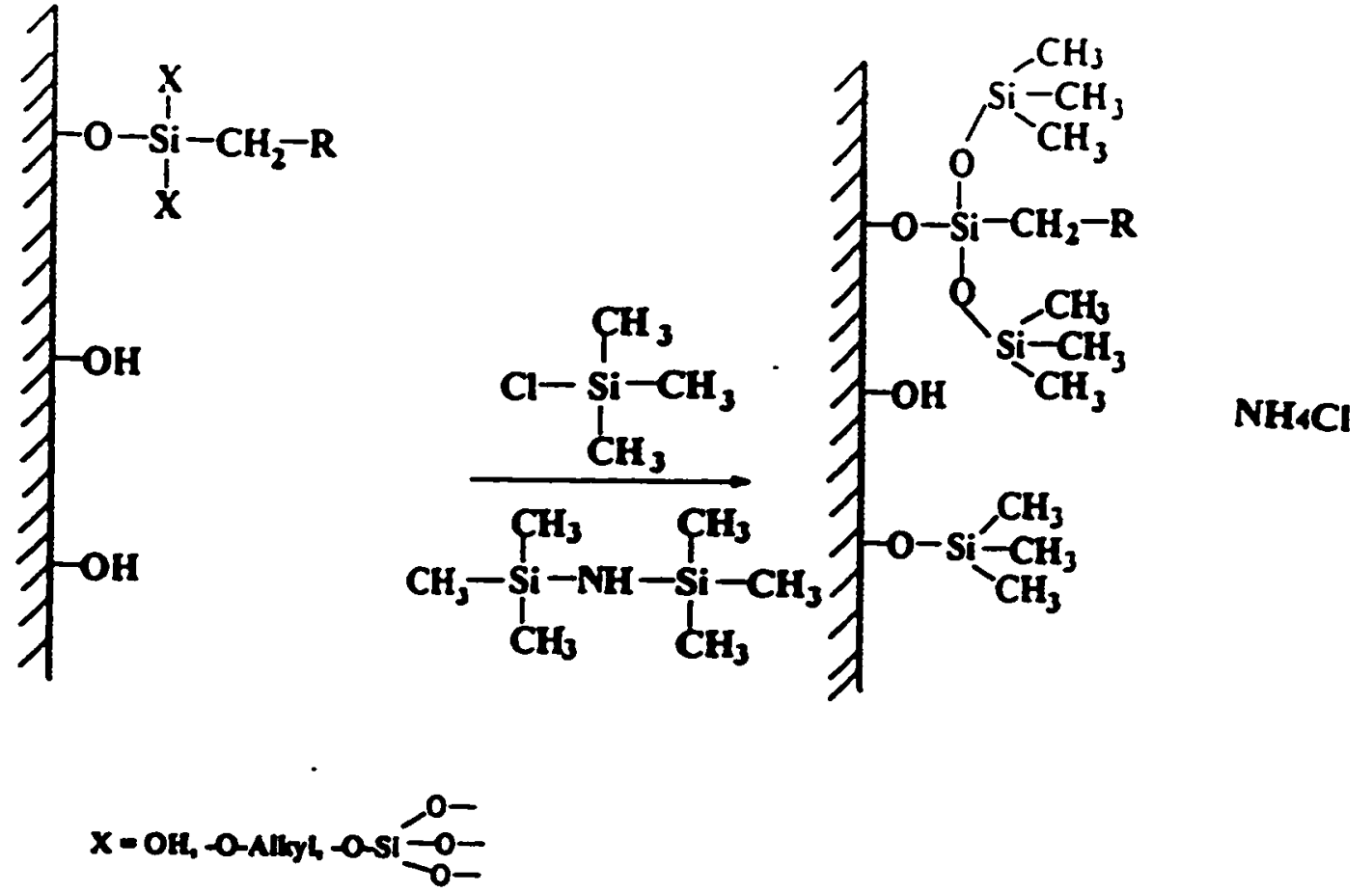

Figure 3. Endeapping reaction scheme by using TMCS and BMDS (1:1 molar ratio). 
advantageous method: TES silanization, followed by hydrosilation, and the addition of TMCS and HMDS (1:1 molar ratio) as the endcapping reagents. However, since this synthetic method is a two step process, two synthetic routes can take place; they are endcapping before bonding (EBB) and bonding before endcapping (BBE). The mechanism is shown in Figure 4. The EBB method involves endcapping of the residual silanols first, and then attachment of $\mathrm{C} 18$ onto the hydride surface. On the another hand, BBE involves attachment of $\mathrm{C} 18$ onto the hydride surface first, and then endcapping residual silanols.

\section{E. Characterization Techniques}

Many techniques have been used to study the nature of both the native silica surface and the bonded silica surface. These techniques can either qualitatively or quantitatively determine the success of bonding of an organic moiety onto the silica surface.

\section{Spectroscopic Techniques}

Spectroscopic techniques are most commonly used to qualitatively determine active groups present on silica surface as well as the successful bonding of certain moieties onto the surface.

\section{1a. Diffuse Reflectance Fourier Transform Infrared Spectroscopy (DRIFT)}

Infrared Spectroscopy (IR) continues to be one of the common spectroscopic techniques for studying functional groups present on a molecule. Since each type of bond (N-H, C-H, O-H, C-O, C=C. etc.) has a different vibration frequency, these finger prints 


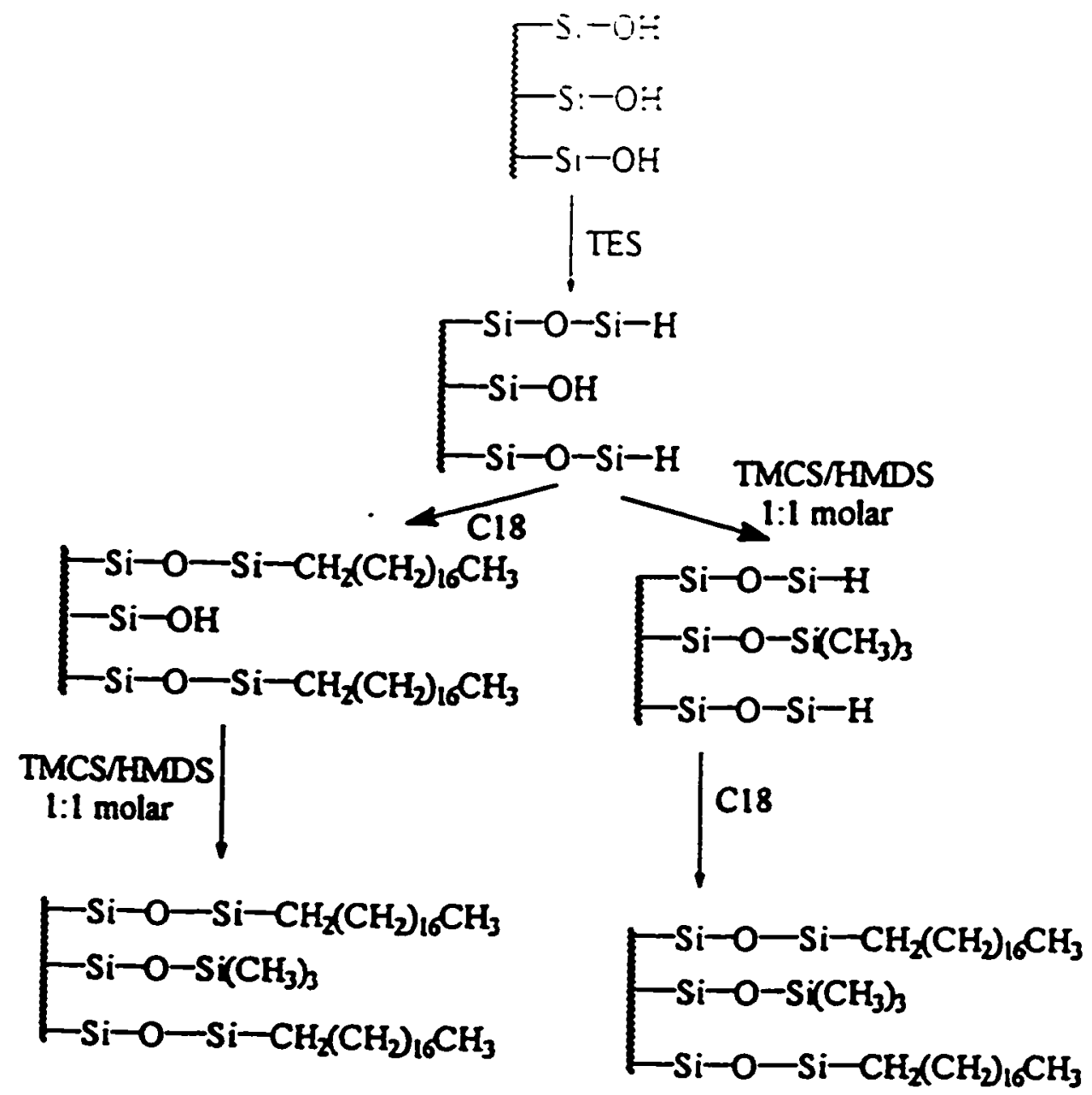

Figure 4. Mechanioms for sgnthesis of endeapped C18 stationary phases. 
of the infrared absorption patterns can give structural information of those compounds. Diffuse Reflectance Fourier Transform Infrared Spectroscopy (DRIFT) has been used to characterize the solid states of both native and bonded phase silica. By mixing a solid sample with $\mathrm{KBr}$ to enhance light throughput, an infrared spectrum of a surface can be obtained. Several typical IR absorptions of a silica surface are as follows: the water absorption can be observed at 4000 to $3000 \mathrm{~cm}^{-1}$, free silanol at $3700 \mathrm{~cm}^{-1}$, Si-H at 2260 $\mathrm{cm}^{-1}, \mathrm{C}-\mathrm{H}$ at $3000-2800 \mathrm{~cm}^{-1}$. By comparing the decrease of silanol peak in the native state, and an increase of $\mathrm{C}-\mathrm{H}$ stretch in the bonded state, one can qualitatively conclude the success of bonding. ${ }^{6,11,12}$

\section{1b. Nuclear Magnetic Resonance (NMR) Spectroscopy}

Nuclear Magnetic Resonance (NMR) is other important tool for identifying and characterizing organic and inorganic compounds. The idea of NMR is that nuclei of atoms absorb electromagetic radiation under a strong magnetic field, and energy level splitting can be induced by the magnetic force. The effect of which nuclei absorb electromagnetic radiation in magnetic fields is related to the molecular structure. ${ }^{12}$ For the past 10 to 15 years, NMR has been used as a powerful technique for identification of the chemical species and measurement of the amount of these species on a surface. The cross-polarization, magic angle spinning NMR (CP-MAS NMR) technique is often used to enhance signal levels for a solid state surface. ${ }^{6}$ Carbon-13 $\left({ }^{13} \mathrm{C}\right)$ NMR and silicon-29 $\left({ }^{29} \mathrm{Si}\right) \mathrm{NMR}$ are the two popular modes for the analyses of silica surfaces. ${ }^{13} \mathrm{C}$ CP-MAS NMR is considered as one of the most useful methods for characterization of modified silica surfaces. By identifying resonances for each of the carbons of the organic moiety 
bonded on the surface, one can positively conclude the success of the bonding reaction. ${ }^{29} \mathrm{Si} \mathrm{CP-MAS} \mathrm{NMR} \mathrm{is} \mathrm{most} \mathrm{useful} \mathrm{for} \mathrm{characterization} \mathrm{of} \mathrm{the} \mathrm{oxide} \mathrm{surface.}{ }^{29} \mathrm{Si}$ CP-MAS NMR has been used extensively for the identification of the active groups on the native silica surface. It can also be used for the bonded phase. By comparing the decrease of $\mathrm{Si}-\mathrm{OH}$ peaks on the native silica surface and the appearance of $-\mathrm{Si}-\mathrm{C}$ peaks on modified surface after an organosilanization reaction, one can also conclude that bonding has occurred.

\section{Elemental analytical methods}

Elemental analyses can serve as a useful method for quantitative determination of carbon surface coverage on a chemically modified surface. The method is mainly done by micro-combustion.

\section{F. Aims of The Work}

First, two ODS stationary phases would be synthesized through TES silanization and hydrosilation reactions. One stationary phase would be endcapped with TMCS and HMDS (1:1 molar ratio) before $\mathrm{C} 18$ bonding (EBB), and the other would be bonded before endcapping (BBE). Spectroscopic techniques including DRIFT and CP-MAS NMR would be used to characterize the success of bonding and endcapping. In addition, the elemental analysis method would be used to calculate surface coverage. Furthermore, chromatographic studies of different organic samples on both columns as well as the commercially made Phenomenex $\mathrm{Cl} 8$ (Pheno $\mathrm{C18}$ ) would be performed. Finally, 
chromatographic studies of temperature and $\mathrm{pH}$ effects on both columns would be performed. 


\section{CHAPTER II}

\section{EXPERIMENTAL}

\section{A. Materials}

\section{Chemicals}

Most chemicals for analysis were purchased from Sigma-Aldrich. Their Chemical Abstract Service (CAS) registry numbers are listed in Table 2.

Table 2: The CAS Registry Numbers of Chemicals Used In this Project

\begin{tabular}{|l|l|}
\hline Chemical Name & CAS Registry Number \\
\hline Trimethylchlorosilane & {$[75-77-4]$} \\
Hexamethyldisilazane & {$[999-97-3]$} \\
1-octadecene & {$[112-88-9]$} \\
Toluene & {$[108-88-36]$} \\
Methylenechloride & {$[74-87-3]$} \\
Methanol & {$[67-56-1]$} \\
Diethyl ether & {$[60-29-7]$} \\
Triethoxysilane (TES) & {$[998-30-1]$} \\
Dioxane & {$[17647-74-4]$} \\
Hydrochloric acid & {$[7647-01-0]$} \\
Tetrahydrofurane & {$[109-99-8]$} \\
Hexachloroplatanic acid (38-40\% Pt) & {$[16941-12-1]$} \\
Anthracene & {$[120-12-7]$} \\
Naphthalene & {$[1146-65-2]$} \\
Potassium Nitrate & {$[57654-83-8]$} \\
o-terphenyl & {$[84-15-1]$} \\
Sodium hydroxide & {$[1310-73-2]$} \\
Pyrene & {$[129-00-0]$} \\
Triphenylene & {$[217-59-4]$} \\
p-toluidine & {$[106-49-0]$} \\
Diphenylamine & {$[122-39-4]$} \\
Pyridine & {$[7291-22-7]$} \\
n,n-dimethylaniline & {$[121-69-7]$} \\
Aniline & {$[62-53-3]$} \\
o-toluidine & {$[95-53-4]$} \\
Trifuoroacetic acid & {$[76-05-1]$} \\
\hline
\end{tabular}




\section{Chemicals for synthesis of endcapped stationary phases}

Endcapping reagents, trimethylchlorosilane (TMCS) and hexamethyldisilazane (HMDS) were purchased from United Chemical Technologies (Briston, PA) . 1octadecene was purchased from Aldrich (Milwaukee, WI). Triethoxysilane (TES) was purchased from Huls-Petrarch Systems. Most of the solvents, toluene (EM Science, Gibstown, NJ), diethyl ether (Fisher Scientific, Fair Lawn, NJ), dioxane (Fisher Scientific, Fair Lawn, NJ) and tetrahydrofuran, THF, (J.T. Baker Chemicals Co. Phillisburg, NJ) were purchased in reagent grade. The silica used for synthesis was VydacTP 106 (The Separations Group, Hesperia, CA) with a particle diameter of 6.5 microns, a pore size of 300 angstroms and a surface area of $106.5 \mathrm{~m}^{2} / \mathrm{g}$.

\section{B. Experimental}

\section{Preparation of Speier's Catalyst}

The preparation of Speier's catalyst was performed in a nitrogen filled glove box. Chloroplatinic acid hexahydrate (Strem Chemicals, Newbury Port, MS) (0.4g) was added to $100 \mathrm{~mL}$ of isopropanol solution at $93{ }^{\circ} \mathrm{C}$. The Speier's catalyst was then stored in a freezer.

\section{Synthesis of Silica Hydride via Silanization}

The synthetic method according to Chu et. al. ${ }^{5}$ was used. Silica was dried prior to any synthesis in a vacuum oven at $110^{\circ} \mathrm{C}$ overnight. In a nitrogen filled glove box, 8.45 $\mathrm{mL}$ of triethoxysilane (TES) $(1.00 \mathrm{M})$ was added to a $50 \mathrm{~mL}$ volumetric flask. Distilled 
dioxane was added up to the mark. The TES/dioxane solution was transferred to a 125 $\mathrm{mL}$ pressure equalizing addition funnel. Next, $10 \mathrm{~g}$ of previously dried Vydac silica was added to a $500 \mathrm{~mL}, 3$-necked round-bottom flask. The 3-necked round-bottom flask was equipped with a West condenser, an addition funnel, a stopper and magnetic bar. Dioxane $(245 \mathrm{~mL})$ was then added to the silica, followed by $10 \mathrm{~mL}$ of $2.3 \mathrm{M} \mathrm{HCl}$. All joints were properly sealed with parafilm. The reaction mixture was heated and refluxed for one hour at $93{ }^{\circ} \mathrm{C}$. Then, the TES was added dropwise to the flask over 20 to 30 minutes. After the TES was completely added, the mixture was heated and refluxed for one hour.

At the end of the reaction time, dioxane was poured off and replaced with toluene. The mixture was centrifuged for 10 minutes. Then the mixture was washed repeatedly three times with a THF/Water mixture $(1: 1 \mathrm{v} / \mathrm{v})$ followed by twice with THF and twice with ether. The wet silica hydride was placed under the hood overnight to evaporate ether. The next day, silica hydride was placed in a vacuum oven and dried at $110^{\circ} \mathrm{C}$ for at least one day.

\section{Synthesis of Bonding Before Endcapping Stationary Phase}

Bonding procedure was followed according to Pesek and Sandoval. ${ }^{13}$ l-octadecene (C-18) $(160 \mathrm{~mL})$ was placed along with $1.2 \mathrm{~mL}$ of Speier's catalyst in a 3-neck roundbottom flask. The mixture was heated and refluxed at 60 to $70{ }^{\circ} \mathrm{C}$ for one hour. Then, 3 $\mathrm{g}$ of Vydac hydride was slowly added to the olefin catalyst mixture, heated and refluxed at $100{ }^{\circ} \mathrm{C}$ for five days. After the reaction time was reached, the mixture was washed three times with toluene followed by three times with methylene chloride and three times with 
ether. After the ether was completely evaporated under a hood overnight, the C-13 bonded silica hydride was dried in vacuum oven overnight. After the solids were completely dried, small portions were used for $\mathbb{R}$ and NMR analysis.

The following endcapping procedure developed by Akapo et. al. ${ }^{14}$ was used. $3 \mathrm{~g}$ of the previously synthesized Vydac-C18 was added into $60 \mathrm{~mL}$ toluene in a three-necked round-bottom flask. $5.625 \mathrm{~mL}$ of TMCS and $9.366 \mathrm{~mL} \mathrm{HMDS} \mathrm{(1:1} \mathrm{molar} \mathrm{ratio)}$ endcapping reagents were added to the reaction flask. The mixture was heated and refluxed at $100^{\circ} \mathrm{C}$ for two hours. After the endcapping reaction time was reached, the mixture was washed three times with toluene, three times with methylene chloride, three times with methanol and water $(1: 1 \mathrm{v} / \mathrm{v})$, followed by three times with methanol. Then, the synthesized BBE was dried in vacuum oven overnight. When it was completely dried, small portions were used for $\mathbb{R}, \mathrm{NMR}$ and carbon analysis.

\section{Synthesis of Endcapping Before Bonding Stationary Phase}

TMCS (5.625 mL) and $9.366 \mathrm{~mL}$ of HMDS (1:1 molar ratio) endcapping reagents were added to $3.0 \mathrm{~g}$ of Vydac hydride in a three-necked round-bottom reaction flask. The mixture was heated and refluxed at $100{ }^{\circ} \mathrm{C}$ for two hours. After the endcapping reaction time was reached, the mixture was washed three times with toluene, three times with methylene chloride, three times with methanol and water $(1: 1 \mathrm{v} / \mathrm{v})$, followed by three times with methanol. Then, the endcapped hydride was dried in vacuum oven overnight. When it was completely dried, small portions were used for IR and NMR analysis.

$160 \mathrm{~mL} 1$-octadecene was placed along with $1.2 \mathrm{~mL}$ of Speier's catalyst in a 3neck round-bottom flask. The mixture was heated and refluxed at 60 to $70{ }^{\circ} \mathrm{C}$ for one 
hour. Then, $3 \mathrm{~g}$ of endcapped Vydac hydride was slowly added to the olefin catalyst mixture, heated and refluxed at $100^{\circ} \mathrm{C}$ for five days. After the reaction time was reached, the mixture was washed three times with toluene followed by three times with methylene chloride and three times with ether. After ether was completely evaporated under the hood overnight, the solids were dried in a vacuum oven. After the solids were completely dried, small portions were used for IR, NMR and carbon analysis

\section{Column Packing}

Both $\mathrm{BBE}$ and $\mathrm{EBB}$ bonded silica were packed individually into two stainless steel columns ( $15 \mathrm{~cm} \times 4.6 \mathrm{~mm}$ internal diameter, Altech Associate Inc., Deerfield, $\mathbb{I L}$ ). A column packer equipped with a pump (Haskell Pump, Burkank, CA) was used for packing the columns. Approximately $1.9 \mathrm{~g}$ of modified silica mixed with carbon tetrachloride and methanol $(9: \mathrm{l} / \mathrm{v})$ was poured into the pump reservoir. The packing was under high pressure by using nitrogen gas. After packing, the column was capped with plastic fittings. Two other short columns ( $50 \mathrm{~mm} \times 4.6 \mathrm{~mm}$ internal diameter) were packed by Higgins Analytical, Inc. (Mountain View, CA).

\section{Characterization Methods}

\section{Diffuse Reflectance Fourier Transform Infrared Spectroscopy (DRIFT)}

DRIFT is one of the major spectroscopic techniques used for surface analysis of bonded materials in this project. DRIFT spectra were taken using a Perkin-Elmer (PE) model 1800 FT-R spectrometer (Norwalk, Connecticut) equipped with a deuterated triglycine sulfate (DTGS) detector. A diffuse-reflectance accessory (Spectra Tech., 
Stamford, CT) with a 2-mm sampling cup was used to hold the solid samples. Pure $\mathrm{KBr}$ was ground using an agate mortar and stored in a desiccator before use. The sample was mixed with ground $\mathrm{KBr}(1: 1 \mathrm{wt} / \mathrm{wt})$. The mixture was then placed in the DRIFT accessory cup, and its surface smoothed using a microscope slide. Then the cup was placed into the sample compartment of the instrument. The compartment was purged with nitrogen gas from a liquid nitrogen gas tank for about 20 minutes to provide a moisture free environment on the surface of the sample. To obtain maximum throughput, alignment of light on the sample surface was adjusted. Pure $\mathrm{KBr}$ was scanned first as reference. All spectra were scanned 100 times at resolution of $2 \mathrm{~cm}^{-1}$ over a region from $4000 \mathrm{~cm}^{-1}$ to $450 \mathrm{~cm}^{-1}$. The signal intensity was then normalized to $100 \%$ transmitance. All signals of the spectrum were analyzed by a Perkin Elmer Series 7500 professional computer and spectra were then plotted by Hewlett Packard 7475A plotter.

\section{Cross-polarization, Magic Angle Spinning NMR (CP-MAS NMR)}

All CP-MAS ${ }^{13} \mathrm{C}$ and ${ }^{29} \mathrm{Si}$ spectra were measued using a Bruker (Billerica, MA) MSL $300 \mathrm{Mhz}$ spectrometer. All samples were loaded into a $\mathrm{ZrO}_{2}$ double bearing rotor and spun at 4700 to $5200 \mathrm{~Hz}$. External glycine and polyhydrosiloxane were used as references for ${ }^{13} \mathrm{C}$ and ${ }^{29} \mathrm{Si}$ spectra. A $5 \mu$ s contact time and a $5 \mathrm{sec}$ repetition rate were used for all signals, and the pulse widths of ${ }^{13} \mathrm{C}$ and ${ }^{29} \mathrm{Si}$ were 6.5 and $5.0 \mu$ sespectively. The probe temperature was kept at $20 \pm 2{ }^{\circ} \mathrm{C}$.

\section{Elemental Analysis}


Samples of BBE and EBB were sent to Desert Analytics (Tucson, AZ) for analysis of carbon percentage. The surface coverage of bonded organic moiety can be calculated through the equation proposed by Berendsen and de Galan:

$$
\alpha_{R}\left(\mu \mathrm{mol} / \mathrm{m}^{2}\right)=\frac{10^{6} \mathrm{Pc}_{c}}{\left(10^{2} \mathrm{M}=\mathrm{n}_{c}-\mathrm{R} \mathrm{M}_{\mathrm{R}}\right) \mathrm{S}_{\mathrm{BET}}}
$$

where $P_{c}$ is the carbon percentage by weight of the bonded phase, $M_{c}$ is the atomic number of carbon, $n_{c}$ is the number of carbons on the bonded organic moiety, $S_{B E T}$ is the specific surface area $\left(\mathrm{m}^{2} / \mathrm{g}\right)$, and $\mathrm{M}_{\mathrm{R}}$ is the molecular weight of the stationary phase.

\section{High Performance Liquid Chromatography}

The HPLC pump was a Waters 515 (Milford, MA). A Waters system multiwavelength uv detector was used to detect all eluting solutes. All columns were purged with $100 \%$ methanol for 10 minutes before use to remove any bubbles inside the tubing. Then, the column was equilibrated. The mobile phase was sonicated for 1 hour to carefully mix remove all bubbles. All solvents used were filtered through a $0.2 \mu \mathrm{m}$ nylon membrane to remove any possible fine particles. All samples injected must be completely dissolved in their solvents. At the end of use of the column for the day, the column was flushed with $100 \%$ methanol for 10 minutes to prolong the column life.

Chromatographic studies of temperature effects on the stationary phase were performed by placing a column inside a thermostat jacket (Fiatron, $\mathrm{CH} 30$ ). The desired temperature was set followed by a 20 minute equilibration time before any sample injections. 
The chromatographic studies of high pH effects on the stationary phase were performed by passing basic mobile phase ( $60 \%$ methanol : $40 \%$ water v/v) at $\mathrm{pH} 10$ through the column. $\mathrm{KNO}_{3}$ was used as the reference, and its elution time was used as the time for one volume of mobile phase to pass through the column. After every 100 volumes of mobile phase passed through the column, a pyrene sample was injected and its chromatogram was obtained. The mobile phase was recycled, and its $\mathrm{pH}$ was adjusted to 10 using $0.1 \mathrm{M} \mathrm{NaOH}$. A similar procedure was used study low $\mathrm{pH}$ effects. The $\mathrm{pH}$ of the acidic mobile phase was adjusted to 2 using concentrated trifluoroacetic acid, TFA, purchased from Pierce Chemical Company (Rockford, Il). 


\section{CHAPTER III}

\section{RESULTS AND DISCUSSION}

\section{A. Elemental Analysis Results}

Elemental analysis is a quantitative method for determining the bonding of chemical materials to the surface. A higher percent of carbon means more carbon loading on the surface, and a high surface coverage $\left(\alpha_{R}\right)$ means more organic moiety has been successfully bonded to the silica surface. Table 3 shows the surface coverage $\left(\alpha_{R}\right)$ and percent carbon results for the two synthesized stationary phases: BBE and EBB. The elemental analysis results clearly indicate that BBE stationary phase is more hydrophobic than EBB.

Table 3. Percent Carbon and Surface Coverage Data Table

\begin{tabular}{|l|l|l|}
\hline $\begin{array}{l}\text { Stationary Phase } \\
\text { Synthesized }\end{array}$ & $\begin{array}{l}\text { Percent Carbon } \\
(w / w)\end{array}$ & $\begin{array}{l}\text { Surface Coverage, } \alpha \\
\left(\mu \mathrm{mol} / \mathrm{m}^{2}\right)\end{array}$ \\
\hline BBE & 6.67 & 3.16 \\
\hline EBB & 4.87 & 2.26 \\
\hline
\end{tabular}

\section{B. Spectroscopic Characterizations of Silica Hydride}

After the TES silanization reaction, DRIFT was first used to characterize the formation of silica hydride. Figure 5, the DRIFT spectrum of Vydac silica hydride, clearly shows the presence of an intense peak at $2250 \mathrm{~cm}^{-1}$. This peak confirms the formation of $\mathrm{Si}-\mathrm{H}$ and success of the reaction. ${ }^{15}$ 


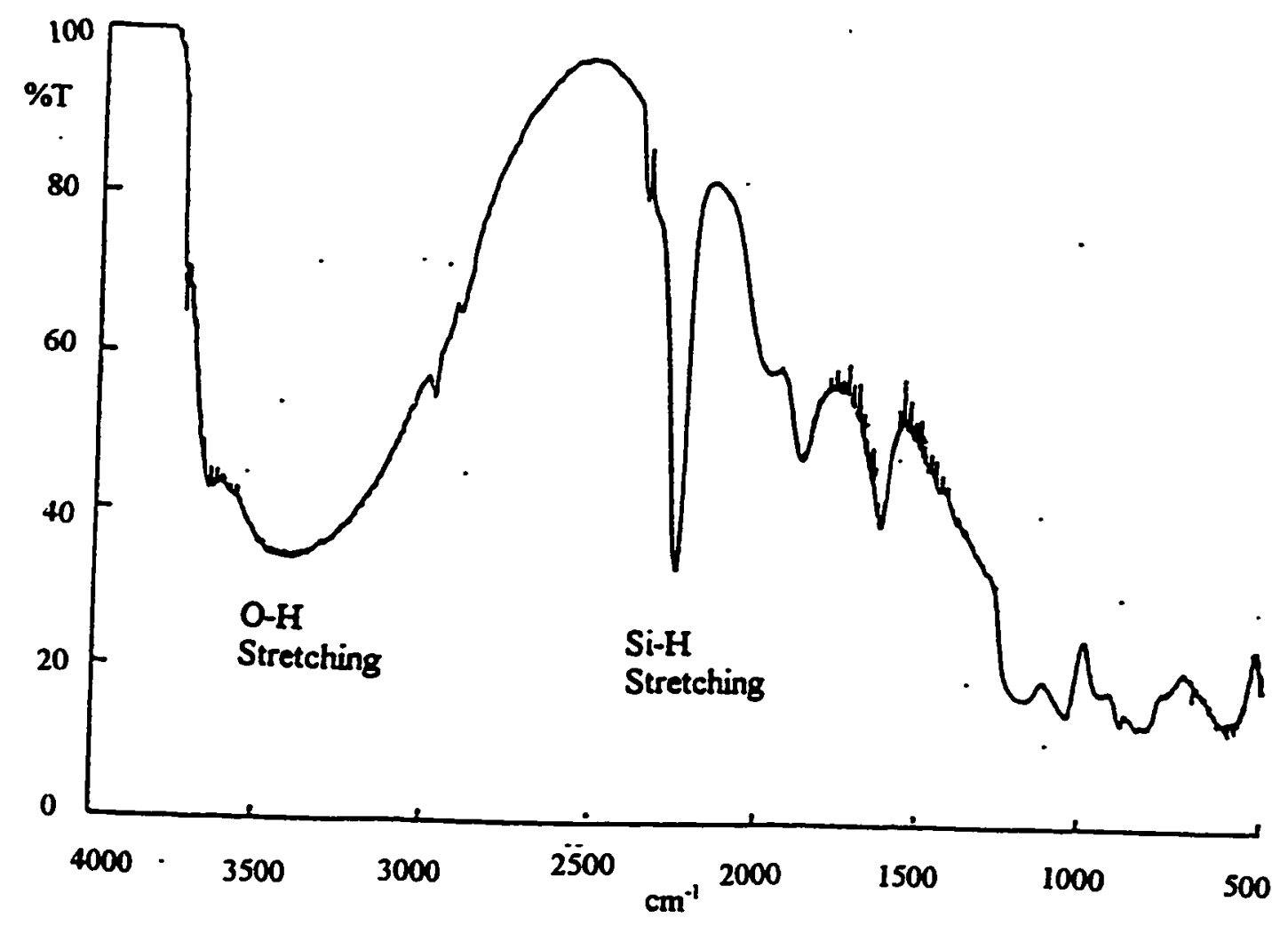

Figure 5. The DRUTT spectrum of Vydac silica hydride. 


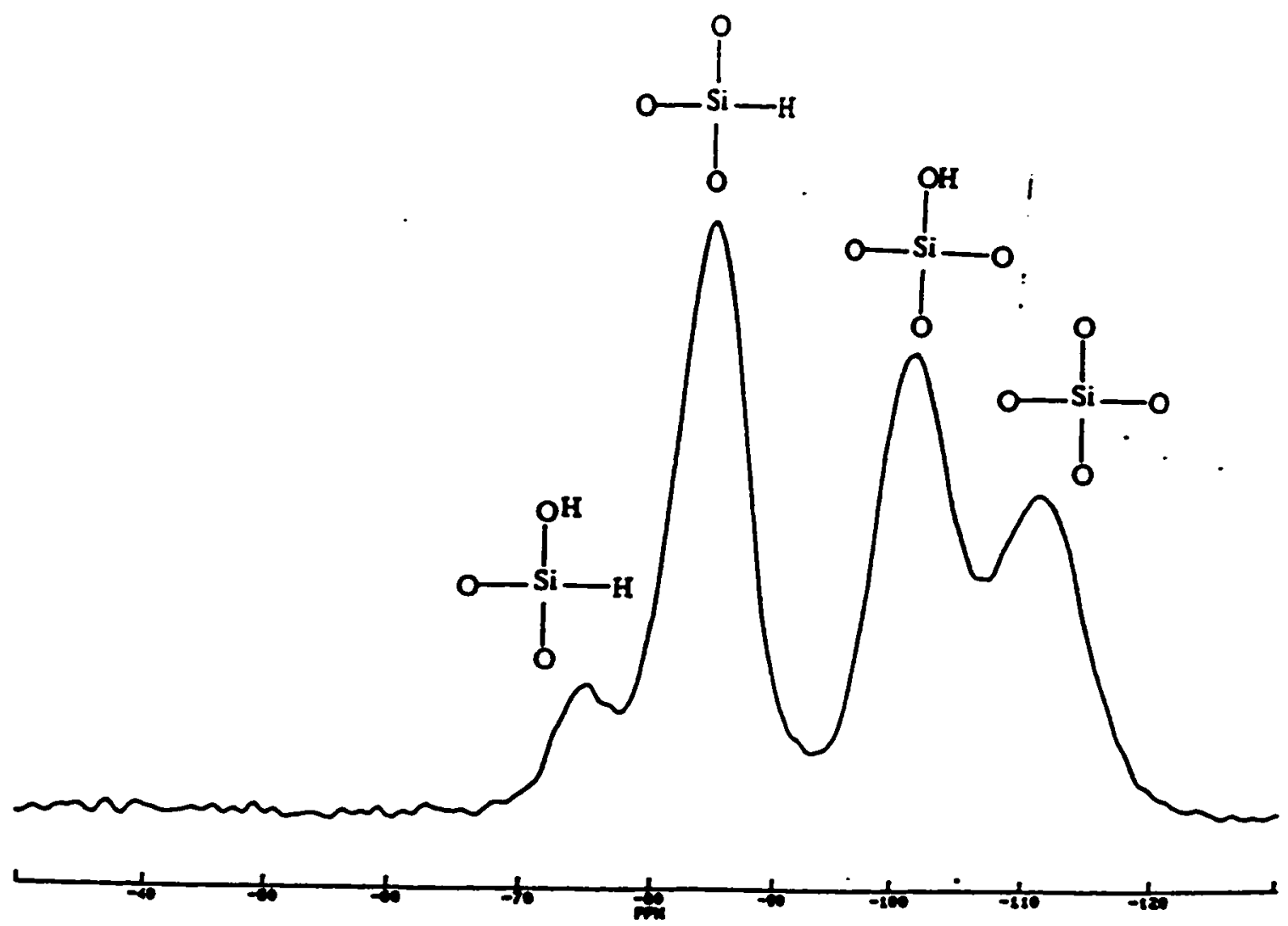

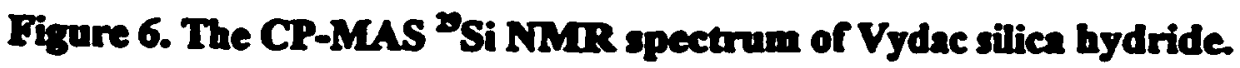


CP-MAS ${ }^{20} \mathrm{Si}$ NMR was also used to monitor the success of the reaction. Figure 6 shows the ${ }^{29} \mathrm{Si}$ NMR of Vydac hydride. The peaks at $-110 \mathrm{ppm}$ and at $-100 \mathrm{ppm}$ represent the backbone of silica. The silicon atom is bonded to four oxygens through siloxane linkages. The peak at $-100 \mathrm{ppm}$ indicates that there are silanols attached to silicon on the surface. The peak at $-85 \mathrm{ppm}$ indicates that there are three siloxane linkages and one hydride. The small peak at $-75 \mathrm{ppm}$ indicates silicon with two siloxane linkages, one hydroxyl group and one hydride. From the evidence of ${ }^{29} \mathrm{Si} N \mathrm{NR}$, along with the $\mathbb{R}$ spectrum, it is confirmed that the hydride intermediate was successfully formed.

Also a CP-MAS ${ }^{13} \mathrm{C}$ NMR was taken and shown in Figure 7. Two peaks are observed in the spectrum. Both of these peaks are due to the carbons from TES. In latter ${ }^{13} \mathrm{C}$ NMR spectra of the bonded phase, these two peaks are eliminated from the spectrum due to the attached organic moieties. ${ }^{13}$

\section{Spectroscopic Characterizations of BBE Bonded Phase}

\section{Spectroscopic Characterizations Vydac Hydride Bonded to C-18}

To synthesize the BBE bonded phase, the olefin, octadecene (C-18), was first reacted with silica hydride to form a monomeric $\mathrm{C}-18$ bonded phase via hydrosilation. Spectroscopic methods such as DRIFT, CP-MAS ${ }^{19} \mathrm{Si}$ NMR and CP-MAS ${ }^{13} \mathrm{C}$ NMR were used to monitor the formation of the C-18 bonded phase. Figure 8 shows the DRIFT spectrum of $\mathrm{C}-18$ bonded to hydride. A significant decrease of the hydride peak at 2500 $\mathrm{cm}^{-1}$ is observed. In addition, intense $\mathrm{C}-\mathrm{H}$ stretching appears at $2800 \mathrm{~cm}^{-1}$. Figure 9 shows the CP-MAS ${ }^{13} \mathrm{C}$ NMR spectrum of $\mathrm{C} 18$ bonded to hydride. The structure 


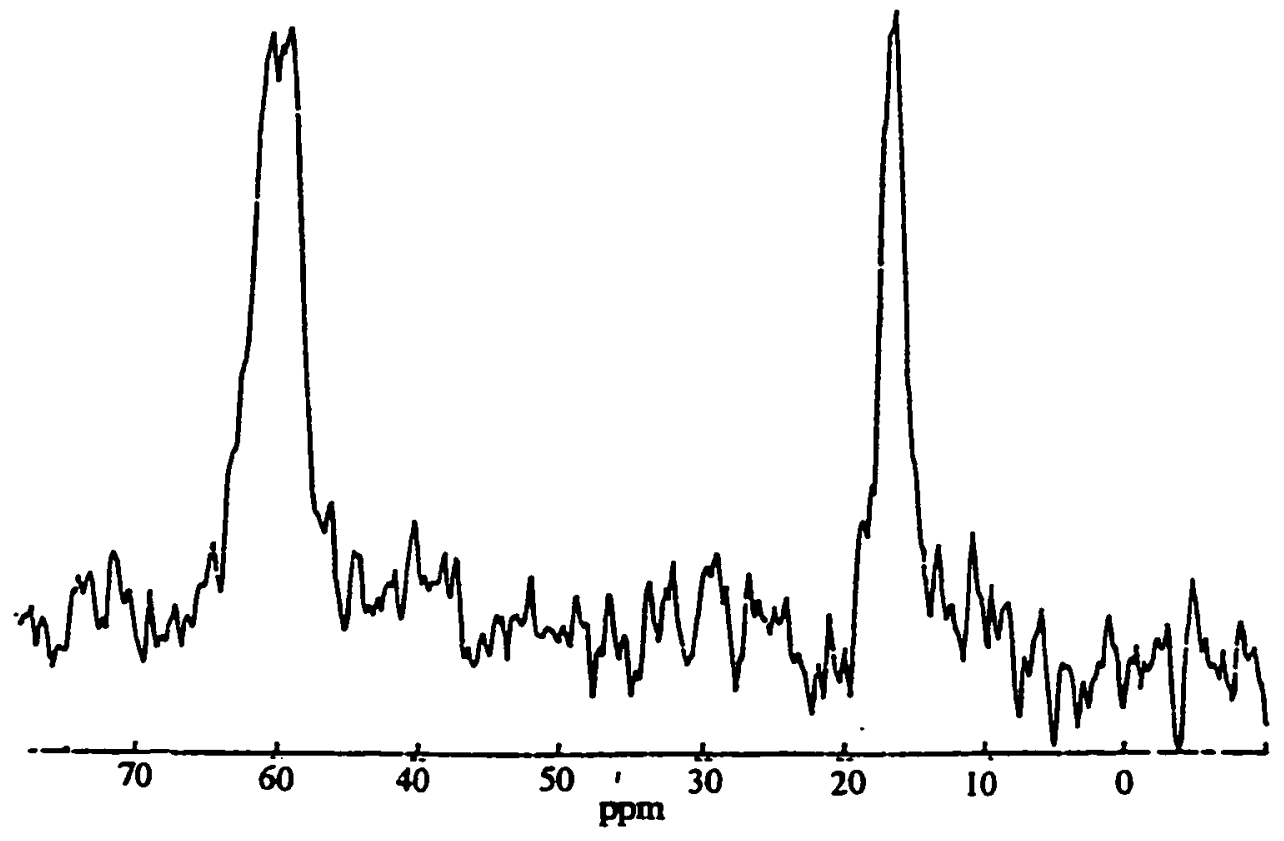

Figure 7. CP-MAS ${ }^{13} \mathrm{C}$ NMR spectrum of Vydac silica hydride. 


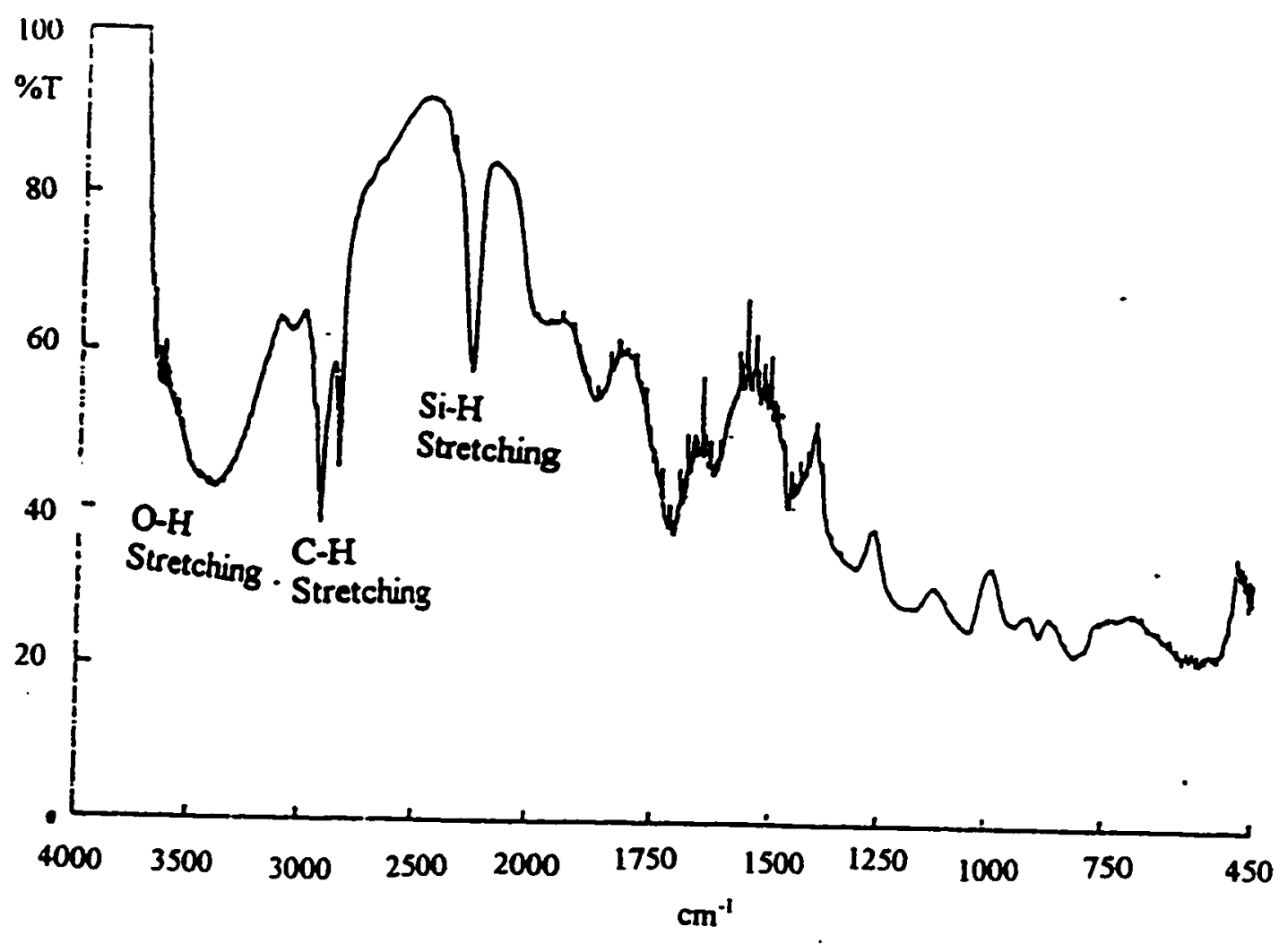

Figure 8. The DRIFT spectrum of C18 Bonded to Vydac hydride. 


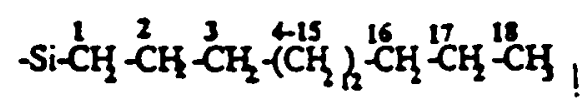

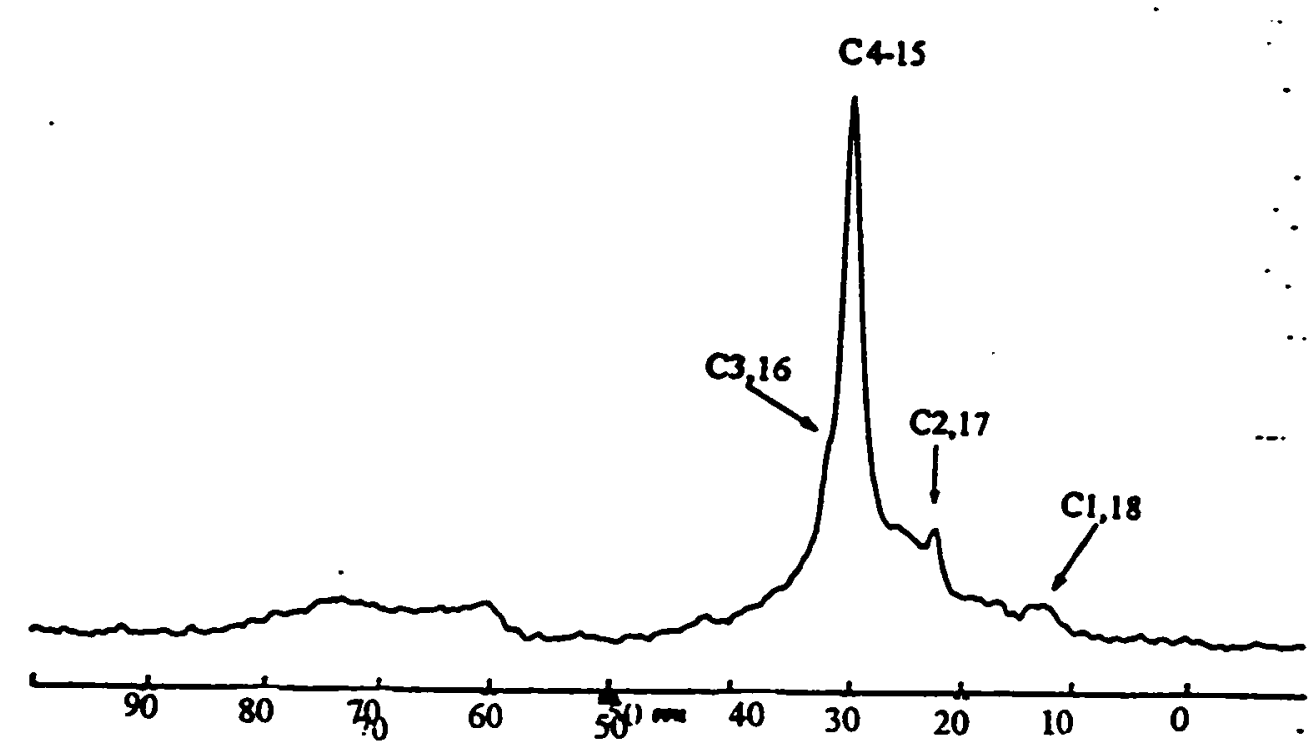

Figure 9. The CP-MAS 'J C NMR spectrum of C18 Bonded to Vydac hydride. 
of the bonded phase is shown, and all relevant carbon peaks are shown and assigned in Figure 9. First of all, an intense peak for $\mathrm{C} 4$ to $\mathrm{Cl} 5$ of the $\mathrm{C}$ - 18 moiety is clearly observed at $30 \mathrm{ppm}$. In addition, a small peak assigned for $\mathrm{C} 1$ and $\mathrm{Cl} 18$ is observed at $14 \mathrm{ppm}$. Furthermore, a peak for $\mathrm{C} 2$ and $\mathrm{C} 17$ appears at $22 \mathrm{ppm}$. Finally, a small shoulder at 32 ppm indicates the $\mathrm{C} 3$ and $\mathrm{C} 16$. Both DRIFT and ${ }^{13} \mathrm{C}$ NMR clearly indicates the bonding of $\mathrm{C}-18$ onto the hydride surface. Thus, it confirms the success of the hydrosilation reaction. $^{4}$

\section{Spectroscopic Characterizations Vydac C-18 Endcapped With TMCS/HMDS}

The second step for synthesis of BBE is the endcapping of the residual silanols on the hydride surface with TMCS/HMDS via a silanization reaction. Figure 10 shows the DRIFT spectrum of Vydac C-18 endcapped with TMCS/HMDS. Compared to the DRIFT spectrum of $\mathrm{C} 18$ bonded to Vydac hydride shown in Figure 8, no significant increase in C-H stretching at $2800 \mathrm{~cm}^{-1}$ and decease in O-H stretching at $3400 \mathrm{~cm}^{-1}$ are observed. Figure 11 shows the CP-MAS ${ }^{29}$ Si NMR spectrum of Vydac C-18 endcapped with HMDS/TMCS. The spectrum shows a significant decrease of hydride peak at -80 $\mathrm{ppm}$ and the appearance of a Si-C peak at $18 \mathrm{ppm}$. Since no ${ }^{29} \mathrm{Si}$ NMR were taken for the sample before endcapping, at this point, it can only confirm the success of C-18 hydrosilation, but not the endcapping reaction. Figure 12 shows the CP-MAS ${ }^{13} \mathrm{C} N M R$ spectrum of Vydac C-18 endcapped with TMCS/HMDS. Compared to the spectrum before endcapping shown in Figure 9, all peaks for 18 carbons of the C-18 moiety appear except the one for $\mathrm{C} 3$ and $\mathrm{C} 16$. This may be due to the overlapping with the intense $\mathrm{C} 4-$ $\mathrm{C} 15$ peak at $30 \mathrm{ppm}$. In addition to the $\mathrm{C} 18$ peaks, one peak appears in this spectrum at 2 


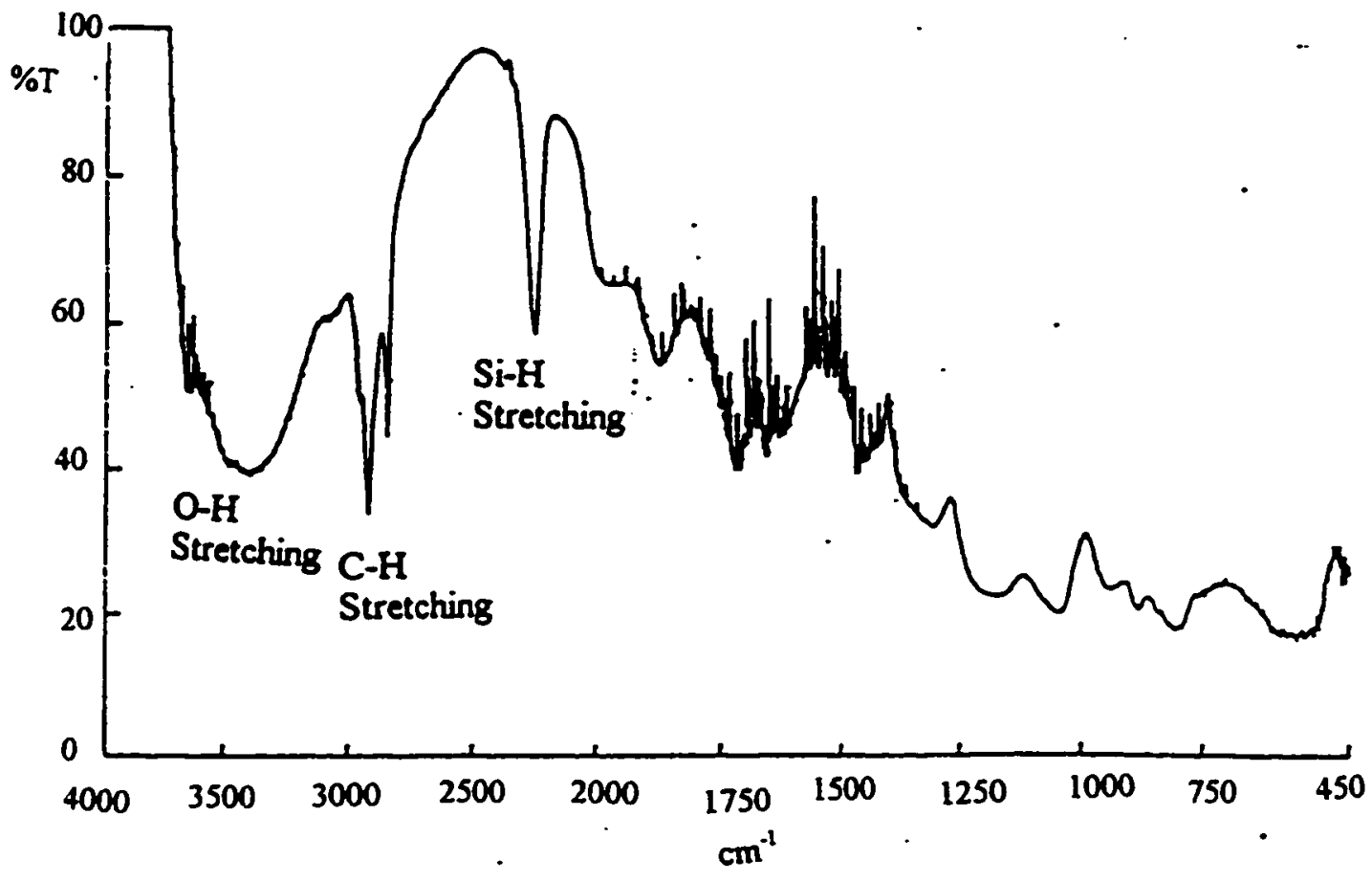

Figure 10. The DRIFT spectrum of Vydac C-18 endeapped with TMCS/RMDS. 


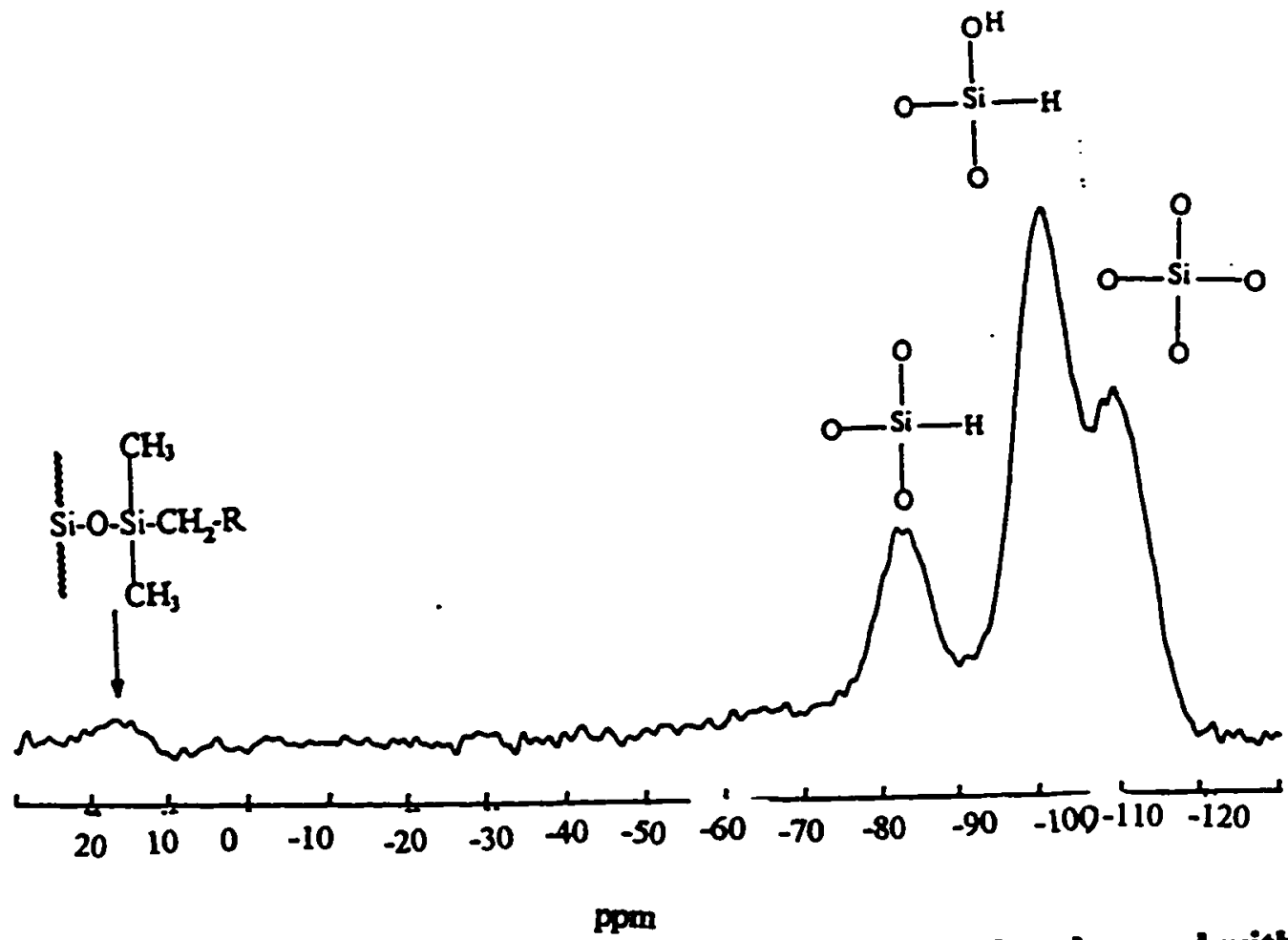

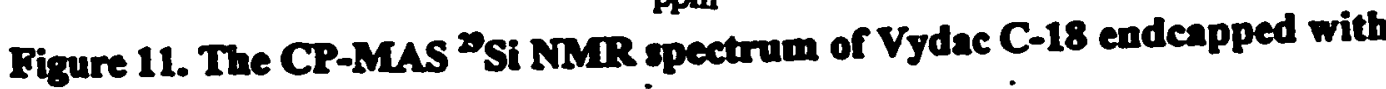
TMCS/RMOS. 


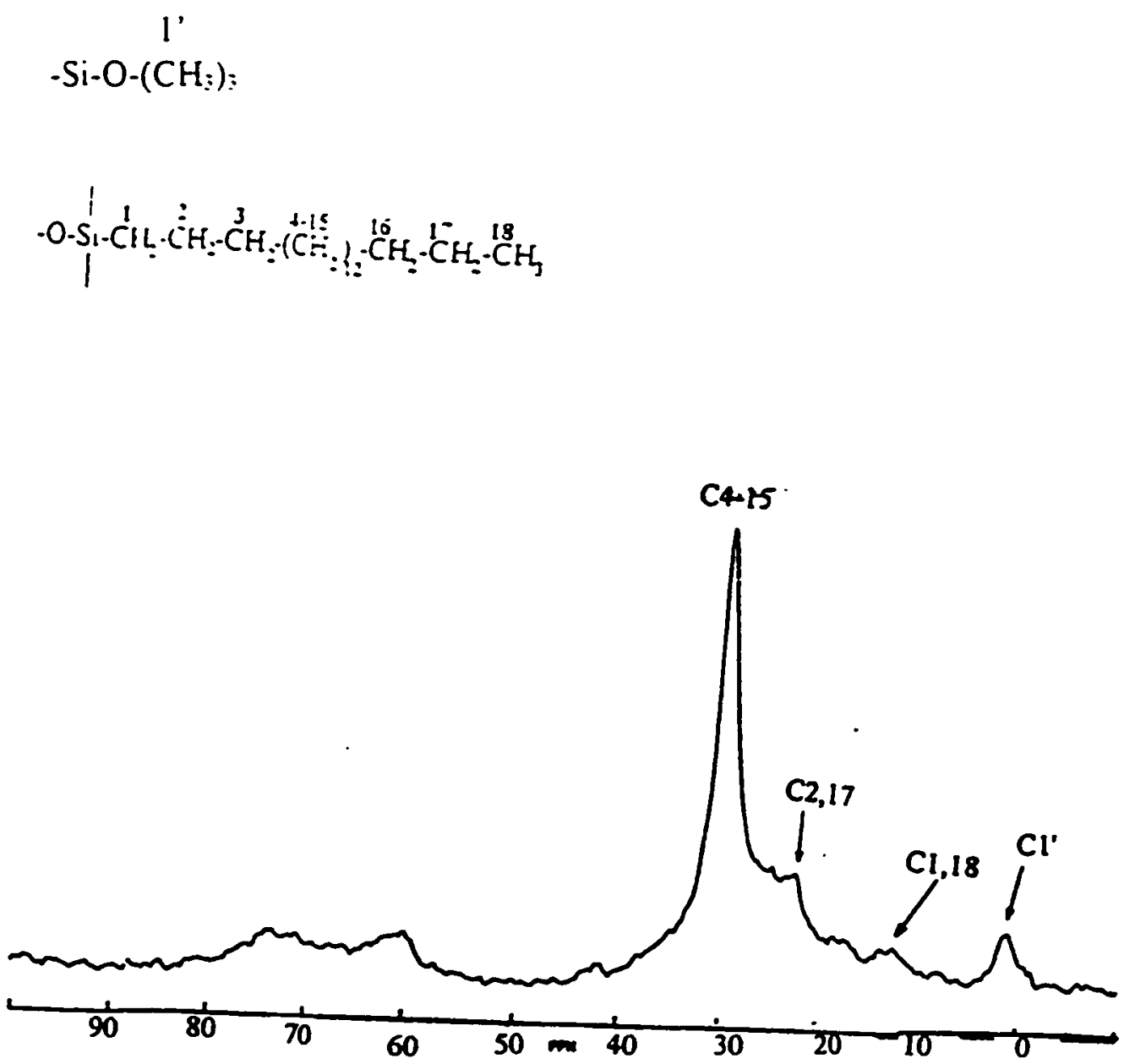

Figure 12. The CP-MAS ${ }^{13} \mathrm{C}$ NMR spectrum of Vydac C-18 endeapped with
TMCS/BMDS. 
ppm. This Si- $\mathrm{CH}_{3}$ peak is clearly due to the endcapping reaction. ${ }^{13}$ Thus, it confirms the success of the endcapping reaction.

\section{Spectroscopic Characterizations of EBB Bonded Phases}

\section{Spectroscopic Characterization of Vydac Hydride Endcapped With}

\section{TMCS/HMDS}

Synthesis of EBB is a two step process. The first step is to endcap residual silanols on the hydride surface using TMCS/HMDS via a silanization reaction. Again, DRIFT and CP-MAS NMR were used to monitor the success of the reaction.

Figure 13 shows the DRIFT of Vydac hydride surface endcapped with TMCS/HMDS. Other than Si-H peak shown at $2250 \mathrm{~cm}^{-1}$ and a O-H peak at $3400 \mathrm{~cm}^{-1}$, $\mathrm{C}-\mathrm{H}$ stretching bands appear between 2800 to $3000 \mathrm{~cm}^{-1}$. These peaks should be due to the endcapping reaction.

Figure 14 shows the ${ }^{29} \mathrm{Si}$ NMR spectrum of the Vydac hydride surface endcapped with TMCS/HMDS. Compared to the ${ }^{29} \mathrm{Si}$ NMR spectrum of Vydac hydride shown in Figure 5 , a Si-C peak appears at $15 \mathrm{ppm}$. This further confirms the success of the endcapping reaction. The final confirmation of endcapping is shown in Figure 15, the CP. MAS ${ }^{13} \mathrm{C}$ NMR of the Vydac hydride surface endcapped with TMCS/HMDS. Other than two peaks at $16 \mathrm{ppm}$ and $60 \mathrm{ppm}$ which are due to carbons from TES, a $\mathrm{Si}-\mathrm{CH}_{3}$ peak appears at $2 \mathrm{ppm}$. From this evidence, we can confirm that trimethylsilyl groups have successfully endcapped some of the residual silanols. It should be noted that not all silanols were completely endcapped by the reaction. 


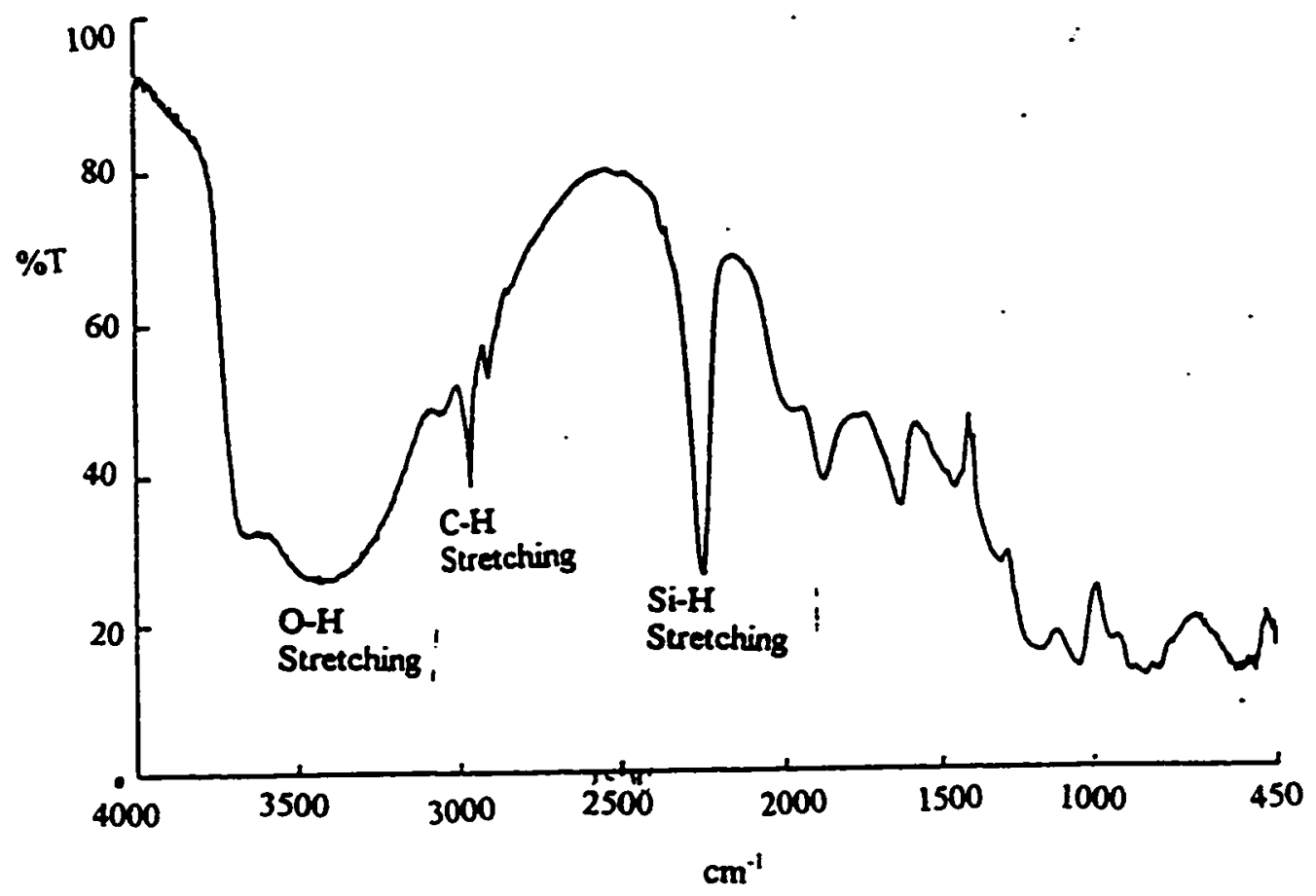

Figure 13. The DRIFT of Vydac hydride endeapped with TMCS/EMDS. 


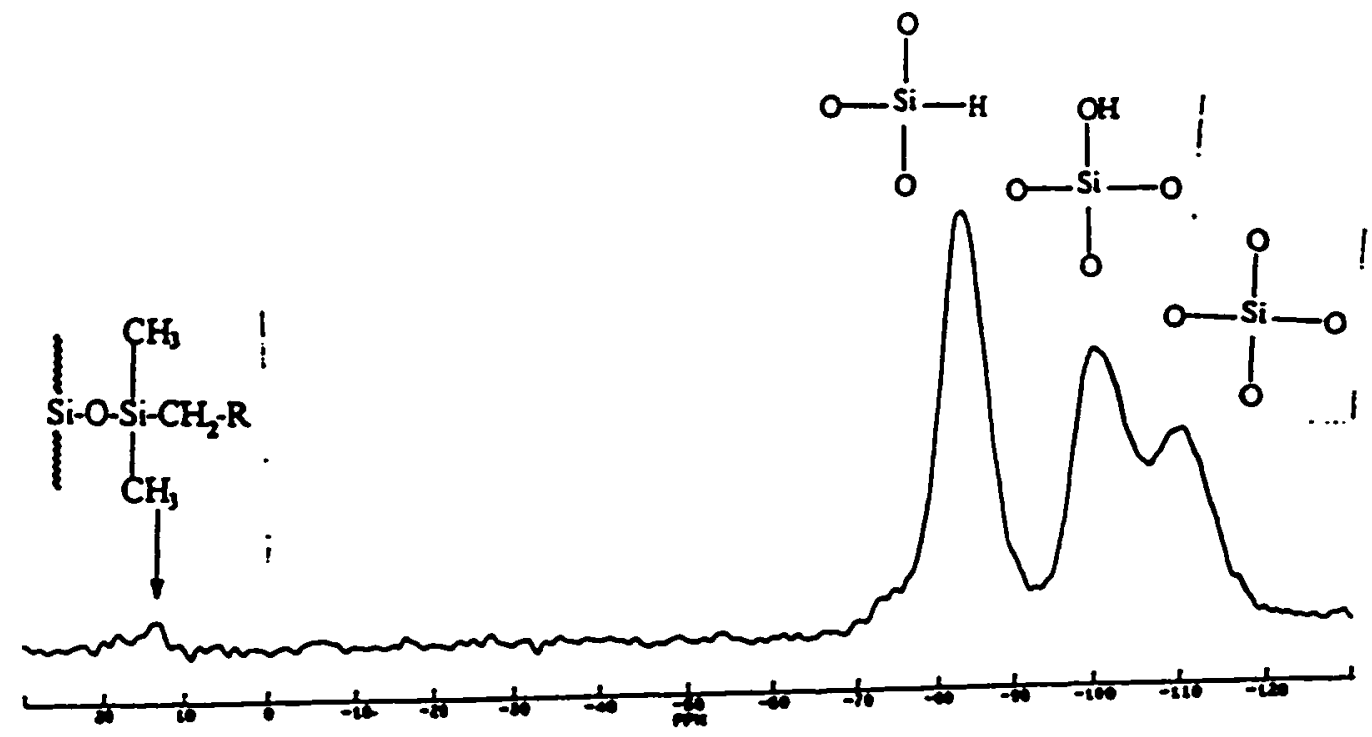

Figure 14. CP-MAS ${ }^{2}$ Si NMR spectrum of Vydac hydride endeapped with TMCShIMDS. 


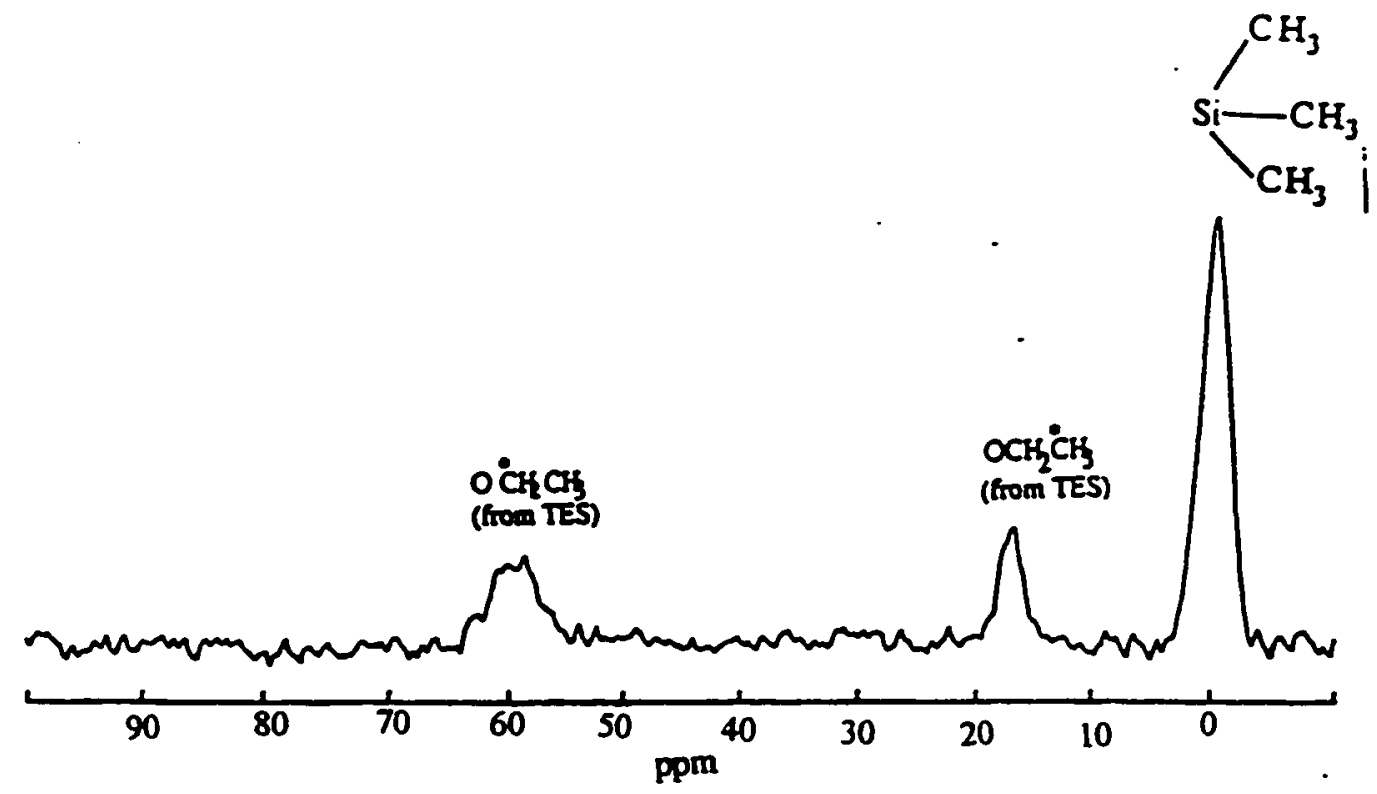

Figure 15. The CP-MAS ${ }^{13} \mathrm{C}$ NMR of Vydac hydride endeapped with TMCS/HMDS (1:1 molar ratio). 


\section{Spectroscopic Characterization of Endcapped Vydac Bonded to C-18}

The second step of synthesing the EBB bonded phase is to attach C-18 to the hydride surface via hydrosilation. Figure 16 shows the DRIFT of endcapped Vydac hydride bonded to $\mathrm{C}-18$. A decrease of $\mathrm{Si}-\mathrm{H}$ stretch at $2250 \mathrm{~cm}^{-1}$ and an increase of $\mathrm{C}-\mathrm{H}$ stretch at $3000 \mathrm{~cm}^{-1}$ are observed. Figure 17 shows the ${ }^{29} \mathrm{Si}$ NMR spectrum of $\mathrm{Cl} 18$ bonded to endcapped Vydac hydride. Compared to the spectrum before $\mathrm{C}-18$ bonding, a significant decrease in the hydride peak at $-78 \mathrm{ppm}$ and an increase of the Si-C peak at 10 ppm are observed. This also qualitatively indicates the success of the bonding. The final confirmation, the ${ }^{13} \mathrm{C}$ NMR spectrum of $\mathrm{C} 18$ bonded to endcapped Vydac hydride, is shown in Figure 18. Other than the endcapping peak at $2 \mathrm{ppm}$, all carbon peaks from $\mathrm{C}$ 18 are observed. They include the peak for $\mathrm{C} 4-15$ at $30 \mathrm{ppm}$, the peak for $\mathrm{C} 2$ and $\mathrm{C} 17$ at $26 \mathrm{ppm}$, the peak for $\mathrm{C} 1$ and $\mathrm{C} 18$ at $18 \mathrm{ppm}$ and the peak for $\mathrm{C} 3$ and $\mathrm{C} 16$ at $32 \mathrm{ppm}$. All of this spectroscopic evidence indicates the success of the $\mathrm{C}-18$ bonding reaction.

\section{E. Chromatographic Evaluation}

Several factors are important when the performance of a column is evaluated. Of cause the column should give good separation of analyte molecules, symmetrical peaks in a chromatogram, low back pressure caused by the stationary phase and high efficiency. In reverse phase chromatography, it is well known that a surface which is more hydrophobic and has low silanol concentration gives better separation and more symmetrical peaks. In this project, several groups of organic compounds have been chosen for chromatographic characterization. Two home-made stationary phases, BBE 


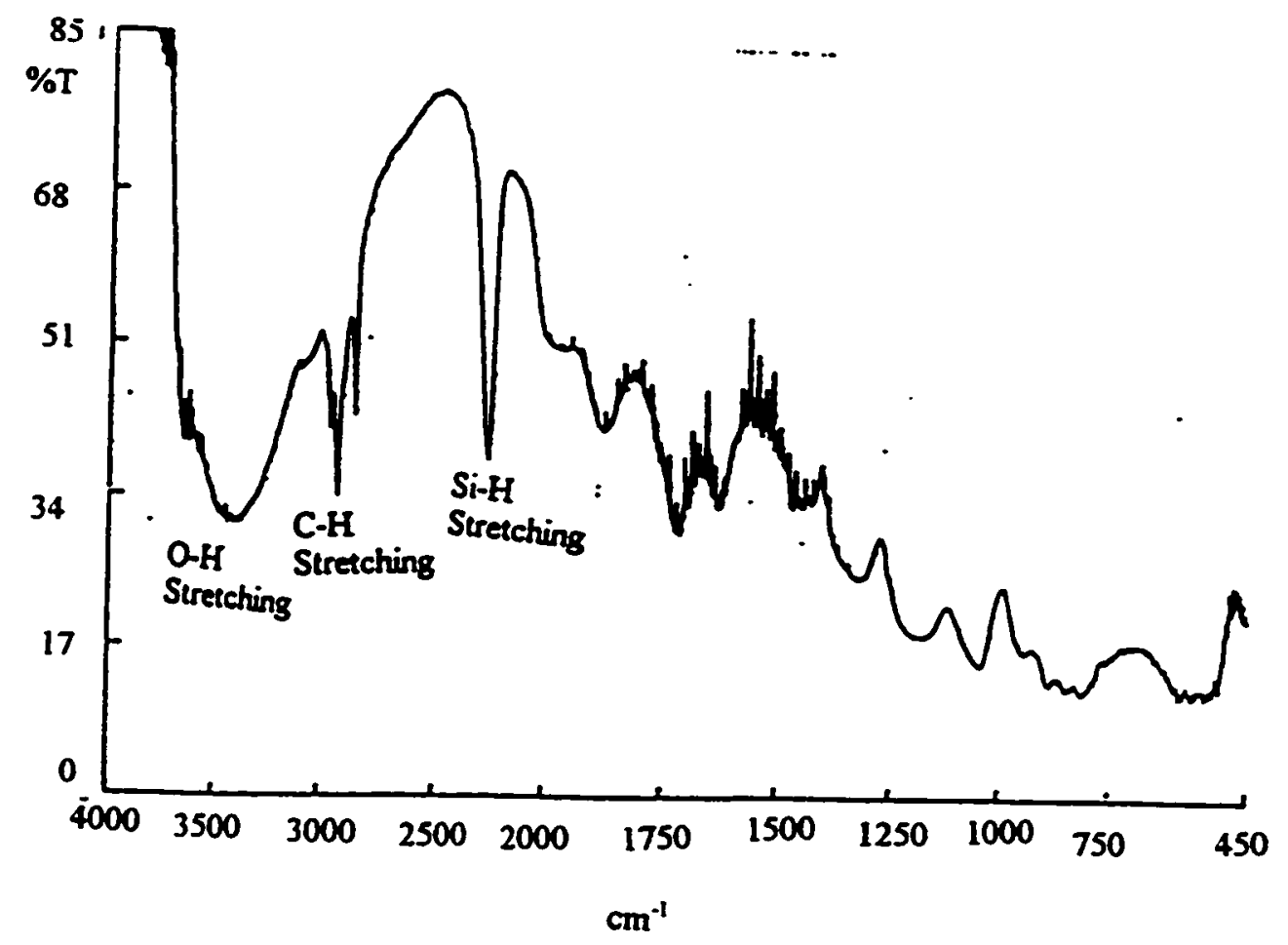

Figure 16. The DRWT of C18 bonded to endeapped Vydac hydride. 


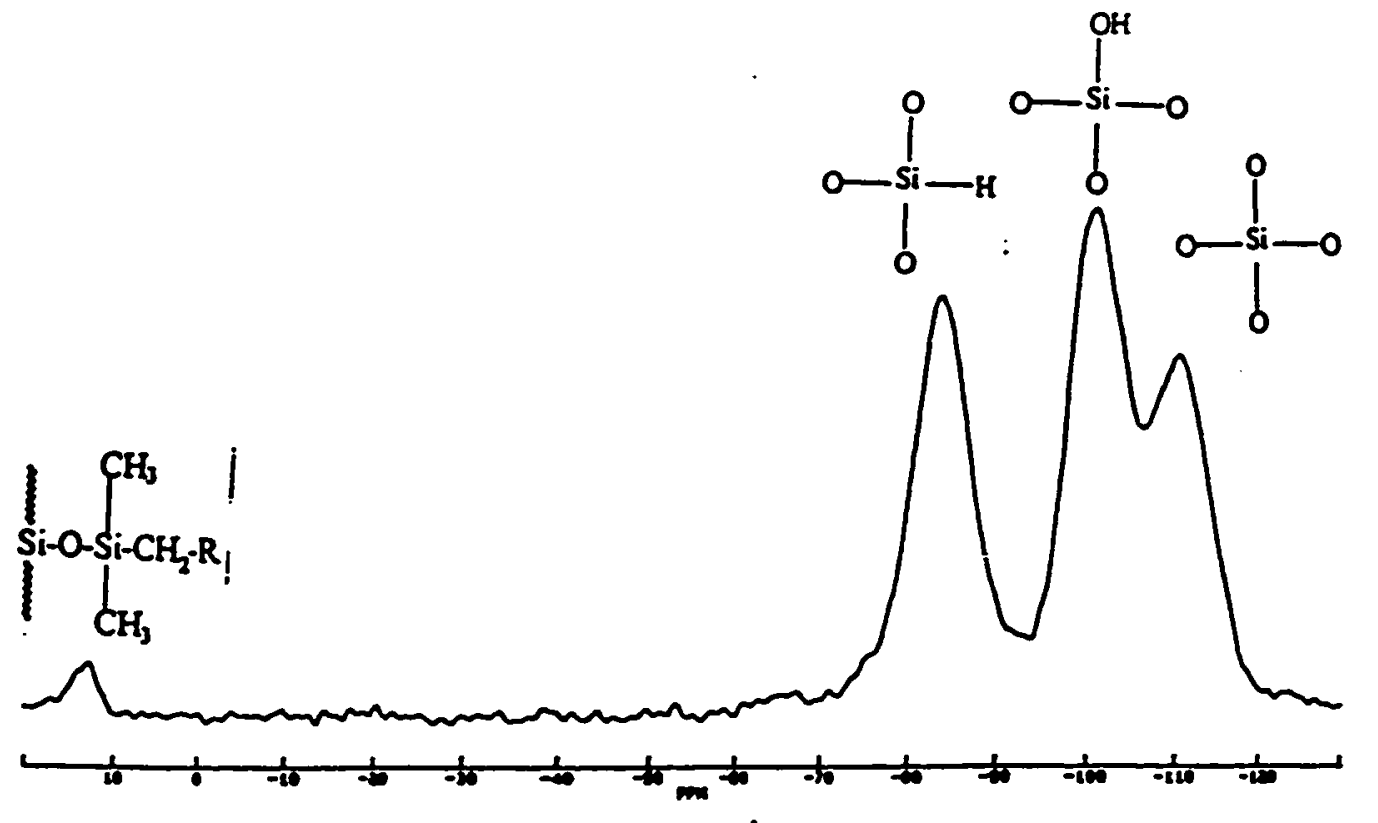

Figure 17. CP-MAS ${ }^{20}$ Si NMIR of C18 Bonded to endeapped Vydac Hydride. 


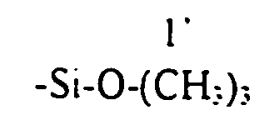

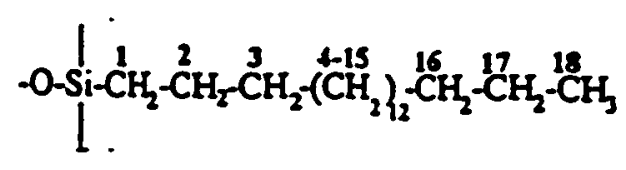

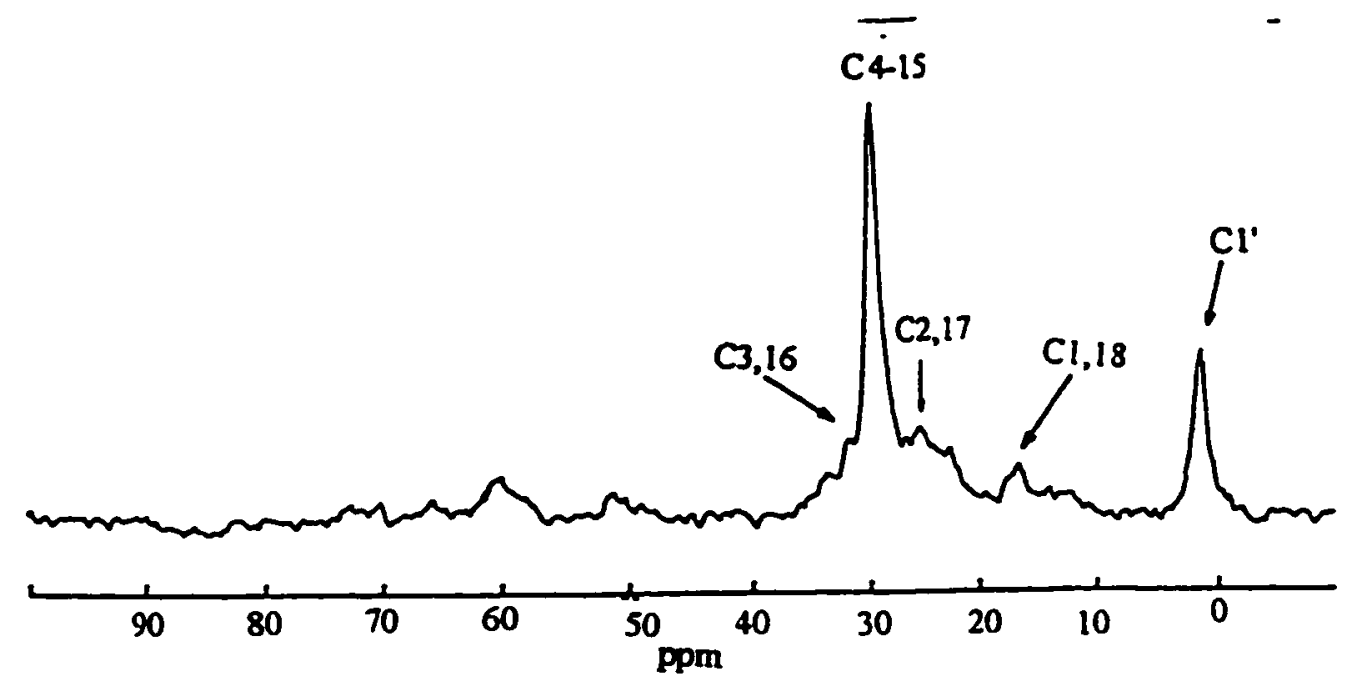

Figure 18. The CP-MAS ${ }^{13}$ C NMR of C18 Bonded to endcapped Vydac hydride. 
and EBB as well as a commercial column, Pheno C18, were studied and compared in terms of their performances. To evaluate separation capability, two test mixtures were used. The first test mixture is called the Perkin Elmer Universal Test Mix (PE-UTM) which consists of six aromatic and non-aromatic compounds. The second test mixture is called Standard Reference Mixture (SRM 869) which consists of three polyaromatic hydrocarbon (PAH) compounds. In addition, several other PAH compounds were also run individually. Finally, several basic organic compounds were run individually to test the peak symmetry or tailing effect.

\section{Isocratic Separation of Perkin Elmer Universal Test Mix}

Perkin Elmer Universal Test Mix (PE-UTM) is a mixture of several compounds; they are sodium chloride, benzene, toluene, ethylbenzene, isopropylbenzene, $t$ butylbenzene and anthracene. In reverse phase chromatography, the elution order is from the most polar compound to the least polar one. The PE-UTM was run on three columns, BBE, EBB and Pheno Cl8 under the same conditions: the same mobile phase $(60 \%$ methanol: $40 \%$ water, $\mathrm{v} / \mathrm{v})$, the same flow rate $(0.5 \mathrm{~mL} / \mathrm{min})$. Separations by the three columns are compared. In order to measure the degree of separation between two components next to each other, resolution $\left(R_{\mathbb{8}}\right)$ between two peaks is normally calculated. $R_{\text {s }}$ is equal to 1.5 when two adjacent peaks are just resolved at the baseline. Figure 19, Figure 20 and Figure 21 show the chromatograms of the separation by the three columns. The R's were calculated and shown in Table 4.

From the results shown in Table 4 , it is observed that separation of PE-UTM by 
Table 4. Resolution $\left(\mathbf{R}_{\mathbf{}}\right)$ for Perkin Elmer Universal Test Mix (PE-UTM) Separated by Three Columns.

\begin{tabular}{|l|l|l|l|l|l|l|}
\hline Column/R & $\mathrm{AB}$ & $\mathrm{BC}$ & $\mathrm{CD}$ & $\mathrm{DE}$ & $\mathrm{EF}$ & FG \\
\hline BBE & 1.47 & 1.60 & 1.7 & 2.59 & 3.27 & 3.52 \\
\hline EBB & 0.88 & 1.13 & 1.4 & 1.23 & 2.07 & 0.2 \\
\hline Pheno C18 & 4.71 & 5.30 & 6.62 & 8.91 & 8.82 & 4.72 \\
\hline
\end{tabular}

BBE is much better than that by EBB. For separation by BBE, except peaks $A$ and $B$, all other peaks were baseline separated. On the other hand, only peak $\mathrm{E}$ and $\mathrm{F}$ were baseline separated by EBB. Excellent separation is observed with P̈heno C18. However, Pheno C-18 gave much higher back pressure and longer retention times due to the smaller particle size.

\section{Isocratic Separation of Standard Reference Mixture (SRM 869)}

Standard Reference Mixture (SRM 869) is also used to test the performance of the three columns. SRM 869 consists of three polycyclic aromatic compounds; they are benzo[a]pyrene (BaP), phenanrhro[3,4-c]-phenanthrene $(\mathrm{PhPh})$ and tetrabenzonaphthalene (TBN). In order to test the performance of the three columns, the same conditions were used; they are $65 \%$ acetoniltrile and $35 \%$ water $(\mathrm{v} / \mathrm{v})$ as the mobile phase and a flow rate of $1 \mathrm{~mL} / \mathrm{min}$. The resulting chromatograms are shown in Figures 22, 23 and 24.

Resolution $\left(R_{s}\right)$ between two adjacent peaks were calculated and are shown in Table 5 .

Table 5. Resolution $\left(R_{s}\right)$ for Standard Reference Mixture (SRM 869) Separated by Three Columns.

\begin{tabular}{|l|l|l|}
\hline Column/R & $\mathrm{AB}$ & $\mathrm{BC}$ \\
\hline $\mathrm{BBE}$ & 1.51 & 4.19 \\
\hline EBB & 1.42 & 1.29 \\
\hline Pheno C18 & 6.36 & 10.01 \\
\hline
\end{tabular}




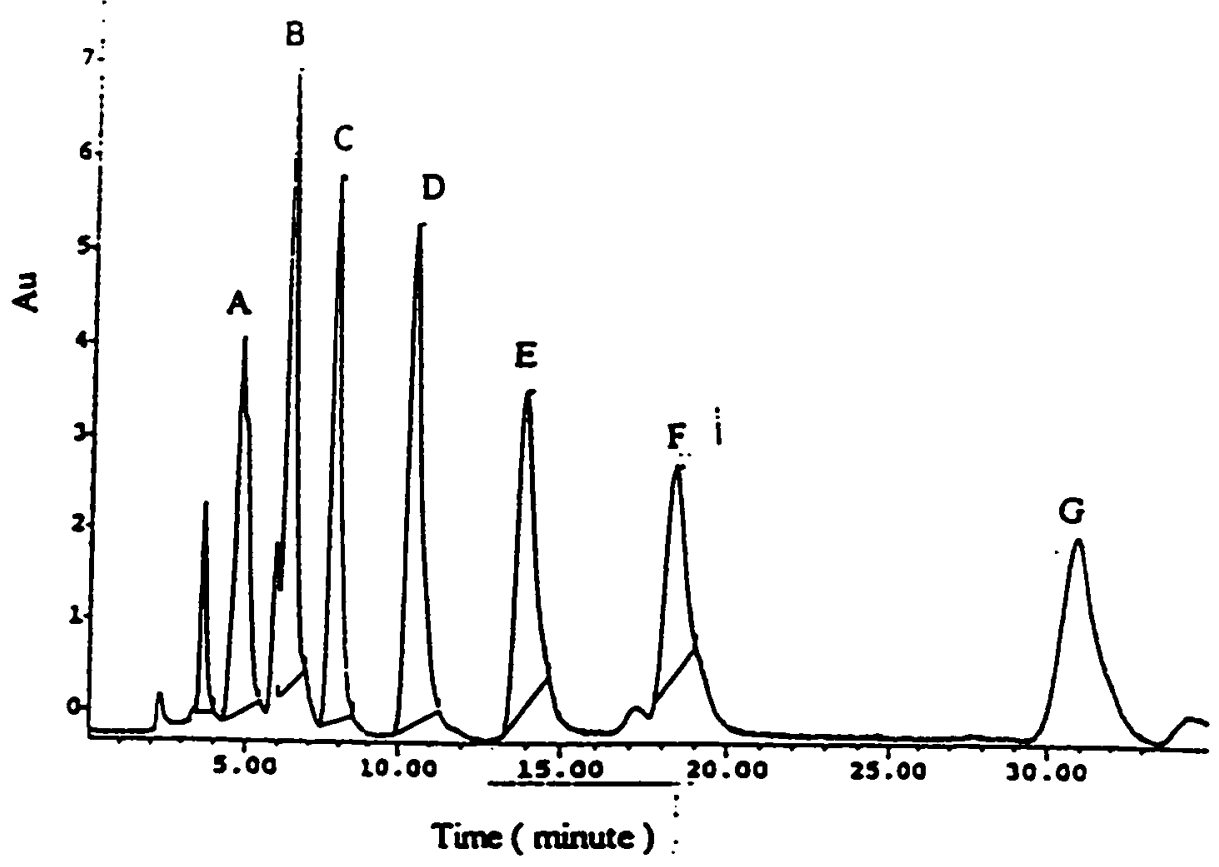

Figure 19. Reverse phase separation of PE universal test mixture on BBE. Mobile phase: $60 \%$ methanol and $40 \%$ water (v/v); flow rate: $0.5 \mathrm{~mL} / \mathrm{min}$; preasure: 55 bar; detection: UV at $254 \mathrm{~nm}$. Peaks: $A=$ sodium chloride; $B=$ benzene; $C=$ toluene; $\mathbf{D}=$ ethylbenzene; $\mathbf{Z}=$ isopropylbenzene; $\mathbf{F}=\mathbf{t}$-butylbenzene; $\mathbf{G}=$
anthracene. 


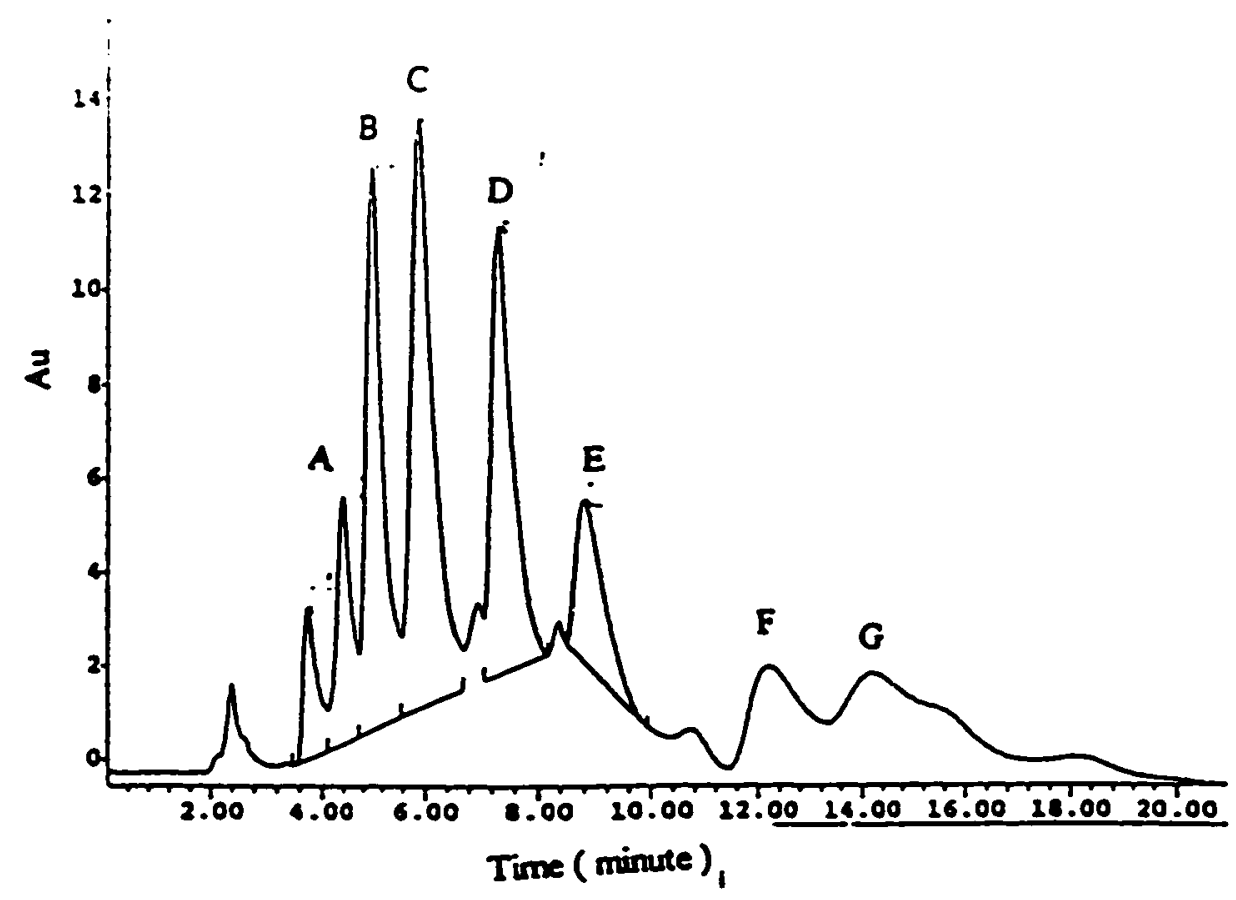

Figure 20. Reverse phase separation of PE universal test mixture on EBB. Mobile phase: $60 \%$ methanol and $40 \%$ water (v/v); flow rate: $0.5 \mathrm{~mL} / \mathrm{min}$; pressure: 35 bar; detection: UV at $254 \mathrm{~nm}$. Pealks: $A=$ sodium chloride; $B=$ benzene; $C=$ toluene; $D=$ ethylbenzene; $\mathbf{E}=$ isopropylbenzene; $\mathbf{F}=t$-butylbenzene; $\mathbf{G}=$ anthracene. 


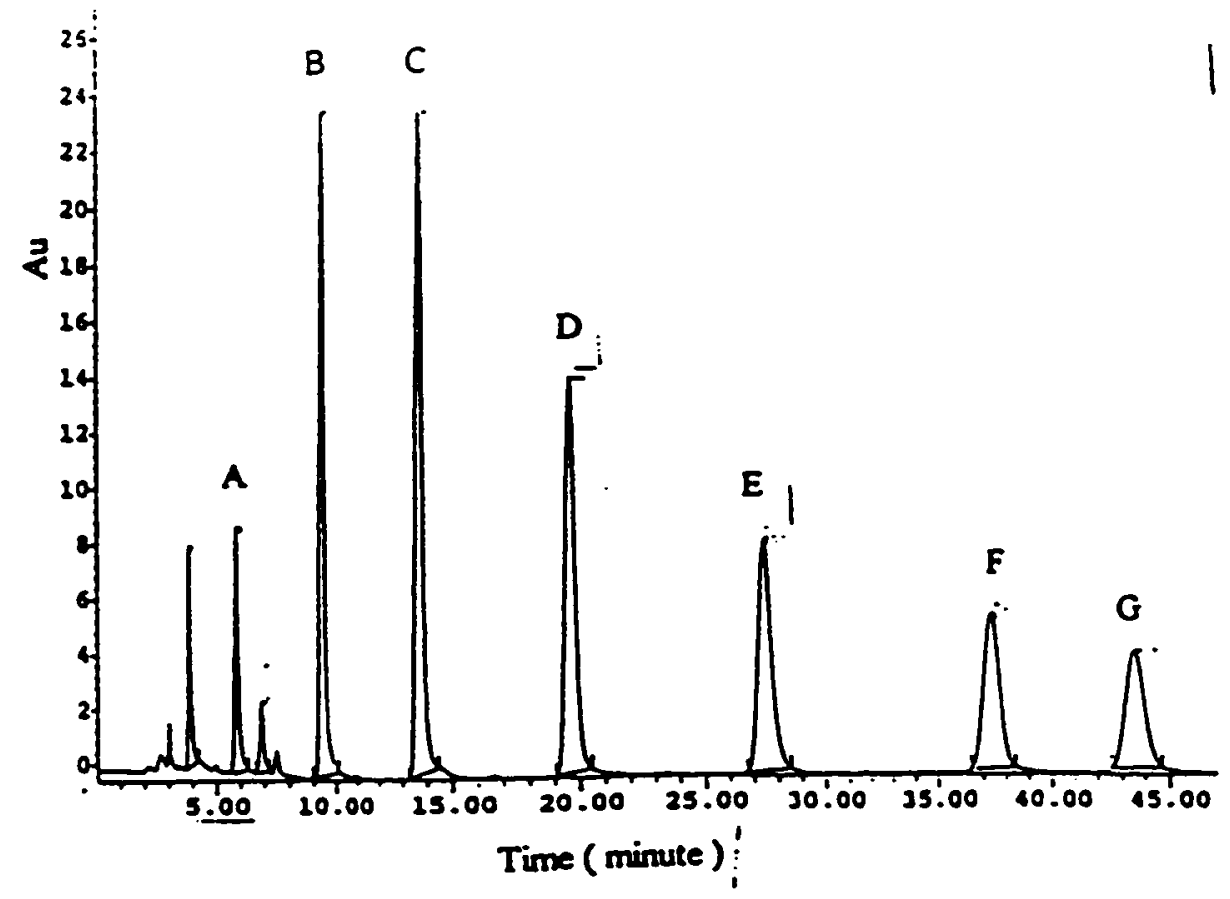

Figure 21. Reverse phase separation of PE universal test mirture on Pheno C18. Mobile phase: $60 \%$ methanol and $40 \%$ water (v/v); fow rate: $0.5 \mathrm{~mL} / \mathrm{min}$; pressure: 85 bar; detection: UV at $254 \mathrm{~nm}$. Pealcs: $A=$ sodium chloride; $B=$ benzene; $C=$ toluene; $D=$ ethylbenzene; $\mathbf{D}=$ isopropylbenzene; $\mathbf{F}=\mathbf{t}$-butylbenzene; $\mathbf{G}=$ anthracene. 
The results shown in Table 5 also indicate that BBE provides better separation compared to EEB. For BBE the three peaks were baseline separated. On the other hand, EBB did not give baseline separation of the three compounds. Again, Pheno C-18 shows much better separation, but with a sacrifice of much higher back pressure and longer retention times. Results shown in Tables 4 and 5 indicate that more hydrophobic interactions are present in BBE, which corresponds to the elemental analysis data in which more carbon is loaded on the BBE surface.

\section{Polycyclic Aromatic Hydrocarbons Compounds}

Polycyclicaromatic hydrocarbons (PAHs) compounds are considered as environmental pollutants produced by incomplete combustion of organic materials. Therefore, separations of PAHs compounds are very important. Since PAHs are nonpolar in nature, the major interaction with the C-18 stationary phase should be hydrophobic. Five PAHs compounds were individually tested to characterize the BBE and EBB stationary phases; they are anthracene, naphthalene, triphenylene, o-terphenyl and pyrene. Their $\log \mathrm{k}^{\prime}$ values versus percentage methanol in water are plotted in Figures $25 \mathrm{a}$ and b. $\mathrm{KNO}_{3}$ was used as the reference compound to calculate the capacity factor ( $\left.\mathrm{k}^{\prime}\right)$. The elution order of these compounds confirms that the major interaction for both columns is hydrophobic. The results also show that the absolute magnitude of retention is directly parallel to the carbon loading on the hydride surface: $\operatorname{BBE}(6.67 \%)>\operatorname{EBB}(4.87 \%)$. This makes sense since more carbon is loaded on the BBE stationary phase which has more hydrophobic interaction than does EBB. 


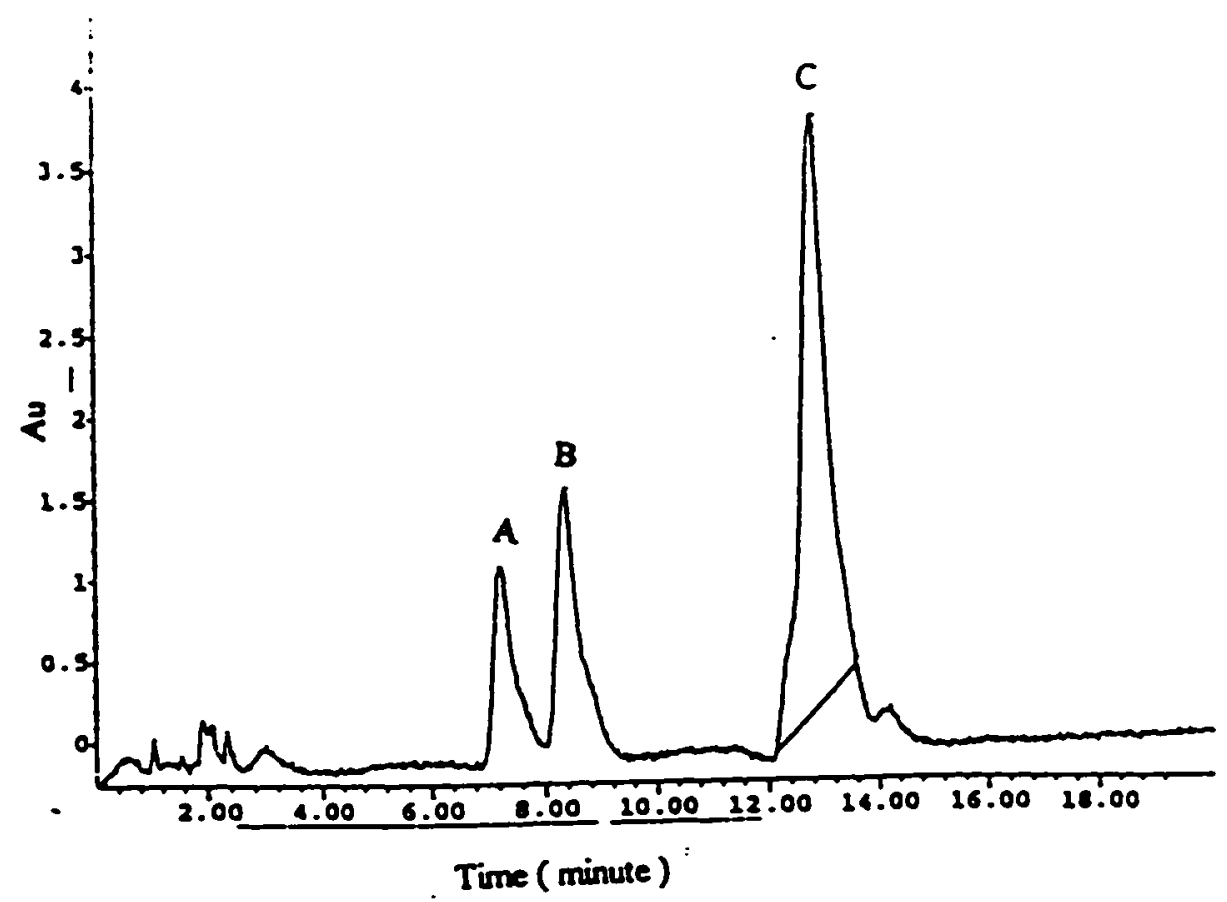

Figure 22. Reverse phase separation of NIST mix (SRM 869) on BBE. Mobile phase: $65 \%$ acetonitrile and $35 \%$ water (v/v); flow rate: $1 \mathrm{~mL} / \mathrm{min}$; pressure: 40 bars; detection: UV 211 nm. Peaks: A = Benzo[a]pyrene; B = Phenanthro[3,4clphenanthrene; $C=$ Tetrabenzonaphthaleace. 


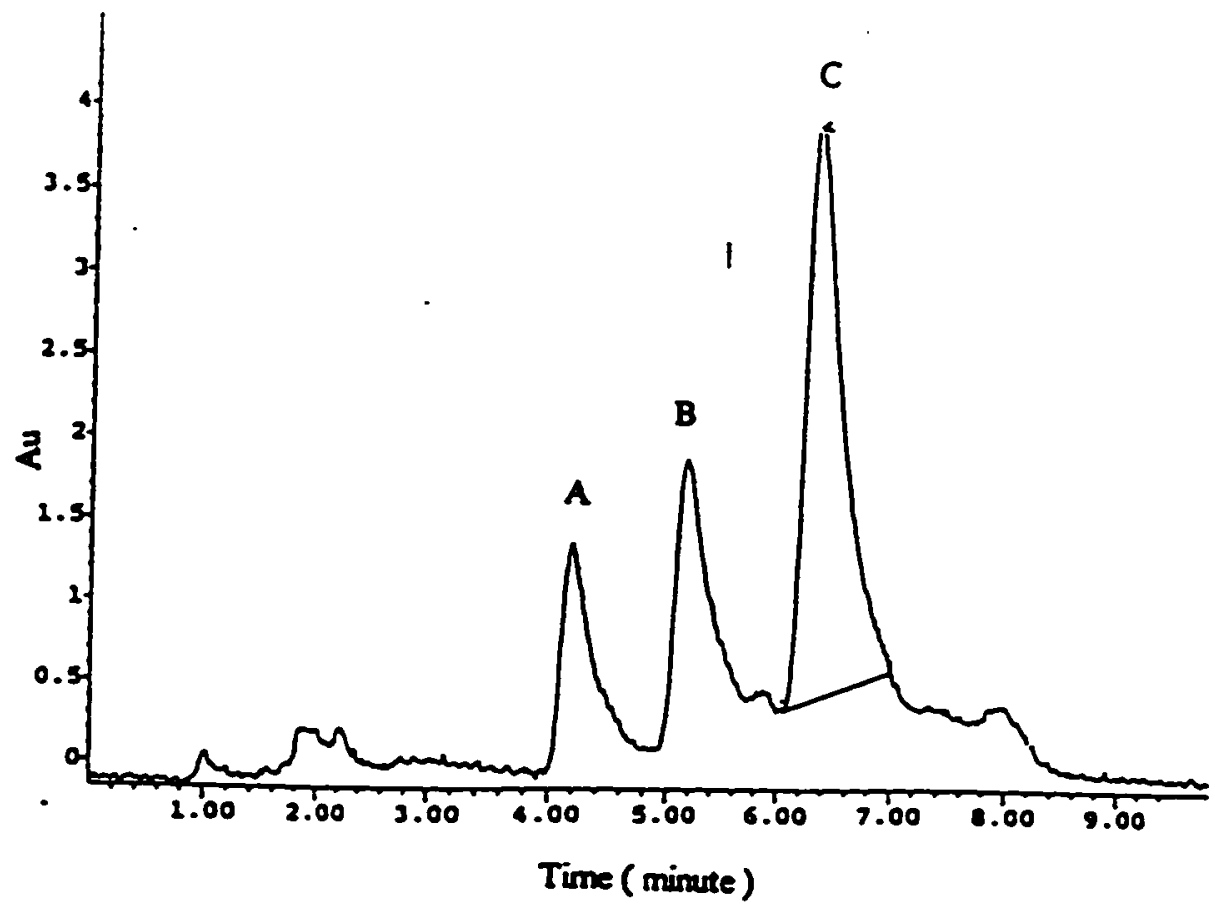

Figure 23. Reverse phase separation of NIST mix (SRM 869) on IBB. Mobile phase: $65 \%$ acetonitrile and 35\% water ( $/ / v)$; flow mate: $1 \mathrm{~mL} / \mathrm{min}$; pressure: 45 bars; detection: UV 211 nm. Peaks: A = Bewoolalpyrenc; B = Phenanthro[3,4ejphenanthrene; $\mathrm{C}=$ Tetrabenzonaphthalene. 


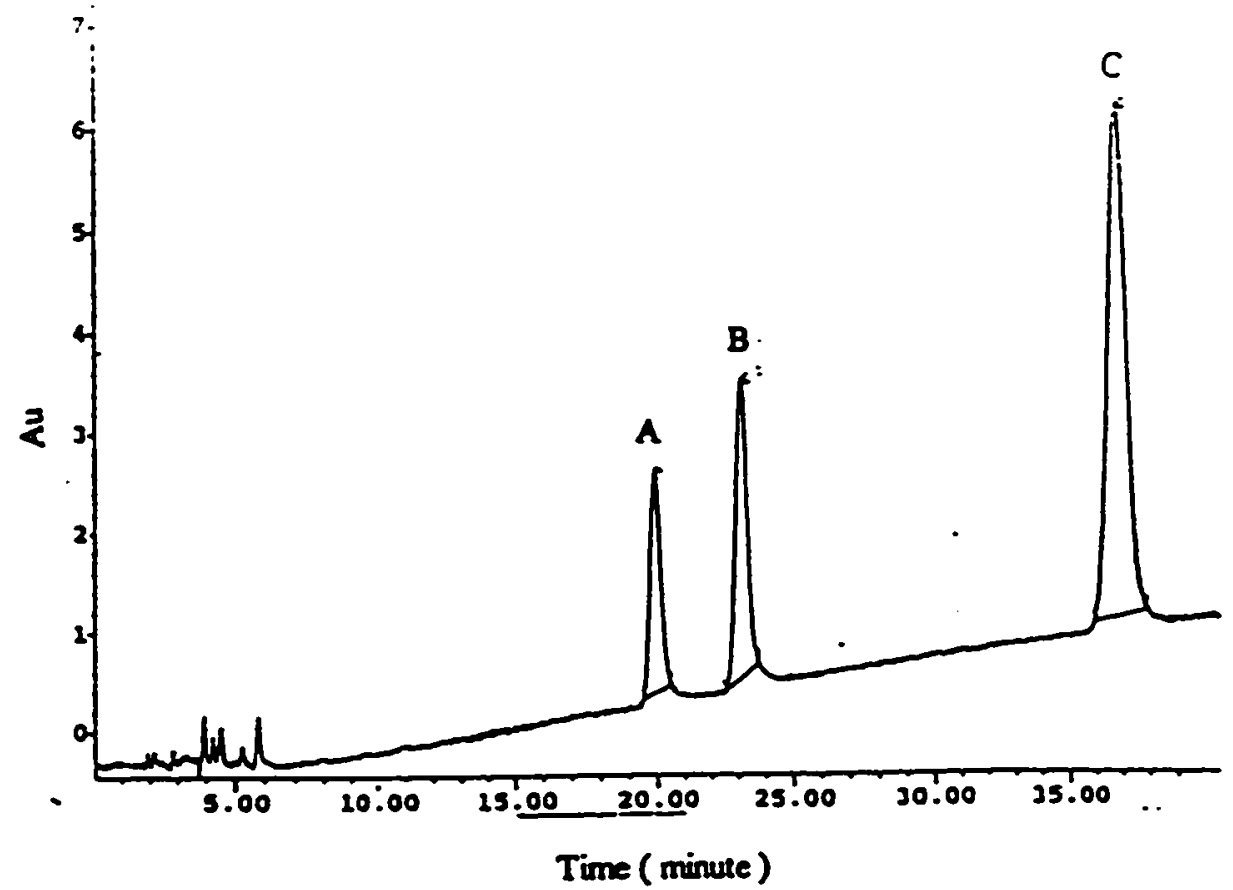

Figure 24. Reverse phase separation of NIST mix (SRM 869) on Pheno C18. Mobile phase: $65 \%$ acetonitrile and 35\% water (v/v); flow rate: $1 \mathrm{~mL} / \mathrm{min}$; pressure: 75 bars; detection: UV $211 \mathrm{~nm}$. Peaks: $A=$ Benzo[a]pyreac; $B=$ Phenanthro[3,4clphenanthrene; $C=$ Tetrabemzonaphthalene. 


\section{Basic Organic Compounds}

Basic organic compounds are those that contain amines or quaternary ammonium compounds. Reverse phase separation of these compounds has always been challenging. As mentioned previously, more than 30 to $40 \%$ silanols (depending on the size of the silane used) can not be bonded during the major modification due to steric effects. Reaction between underivatized silanols and the basic compounds occurs through an ionexchange process. Such a process leads to peak tailing or band broadening in a chromatogram so that inferior separation occurs. ${ }^{8}$ As mentioned previously, endcapping is a method that can shield some of the residual silanols on the surface after the primary modification. Better endcapping leads to less silanols on the surface, thus better peak shapes and less tailing.

Two basic compounds, p-toluidine and pyridine, were used to test tailing effects on BBE, EBB and Pheno C18. The results are shown in Figures 26 (a) and (b). Comparing $\mathrm{BBE}$ to $\mathrm{EBB}$, it is observed that $\mathrm{BBE}$ gives less tailing. This is a clear indication that BBE is a better endcapped stationary phase and has less silanols on the surface. Comparing BBE and EEB to the commercially made Pheno C18, it is observed that $\mathrm{BBE}$ is equal or better. Also, from the results, it is observed that in general as the methanol percent in the mobile phase increases, the peak shape becomes better. This is probably because the solute molecules elute faster in higher percentage methanol, thus the peak becomes narrower and sharper. As a result, tailing becomes less significant. Sample chromatograms are shown in Figure 27. 


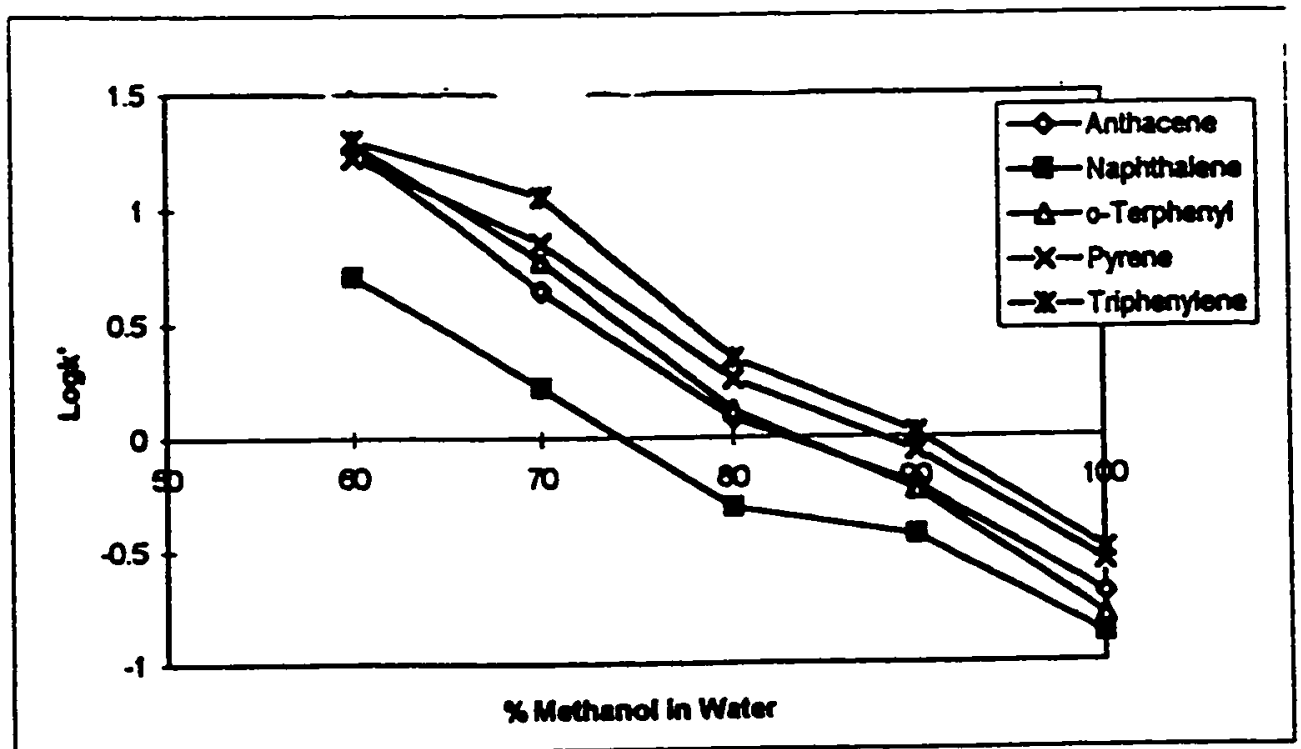

Figure 25 (2). Chromatographic studies of PAH compounds on BBE. 


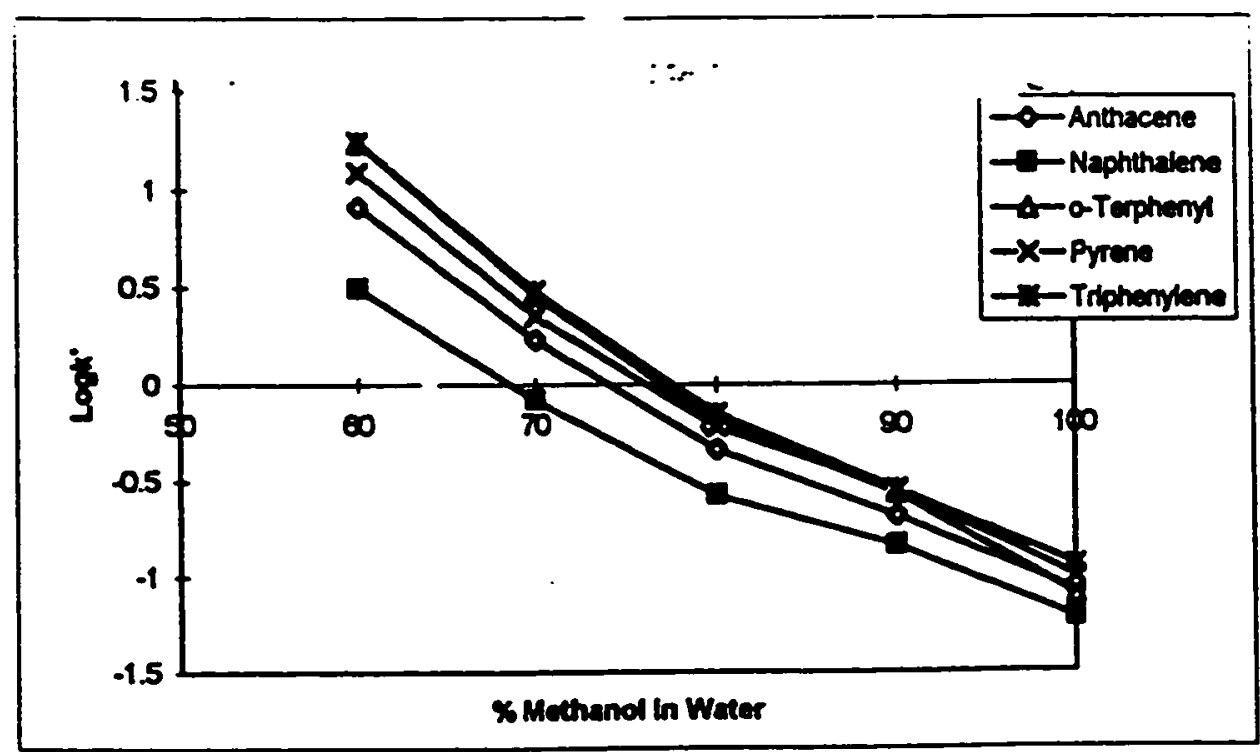

Figure 25 (b). Chromatographic studies of PAA compounds on EBB. 


\section{G. Chromatographic Study on Temperature Effect}

As the results show in the study of basic compounds, mobile phase content can affect tailing. At higher organic mobile phase percent, there is less tailing. Another parameter that may affect peak shape is temperature. Chromatographic studies of temperature effects on $\mathrm{BBE}$ and $\mathrm{EBB}$ were also performed. Figures 28 (a) and (b) show that with the same mobile phase, when temperature is increased, retention is decreased Figures 29 (a), (b) and (c) show the trend in the asymmetry factor as temperature increases. The result indicates that in general, as temperature increases, peak shape becomes slightly improved. There may be two reasons. First, kinetically, when temperature increases, the rate of mass transfer increases. Therefore, solutes elute faster. As a result, a peak becomes sharper, and the shape is improved. Also, thermodynamically, at higher temperature, solutes have higher energy to interact with stationary phase, thus the selectivity increases. Therefore, increasing temperature can improve peak shape, thus give better separation.

\section{H. Chromatographic Studies on pH Effects}

\section{High pH Stability Study}

It is well known that a silica-based bonded phase is stable only in a $\mathrm{pH}$ range between 2 to $7.5 .^{16}$ At low $\mathrm{pH}$, the siloxane linkage, Si-O-Si, is hydrolytically unstable. At a pH above 7.5 , the bonded phase tends to be dissolved in alkaline solution. It is known that the bonded phase does not dissolve in alkaline solution, but silicate does. ${ }^{16,17,18}$ The dissolution of silicate may lead to destruction of the silica support. It has been 


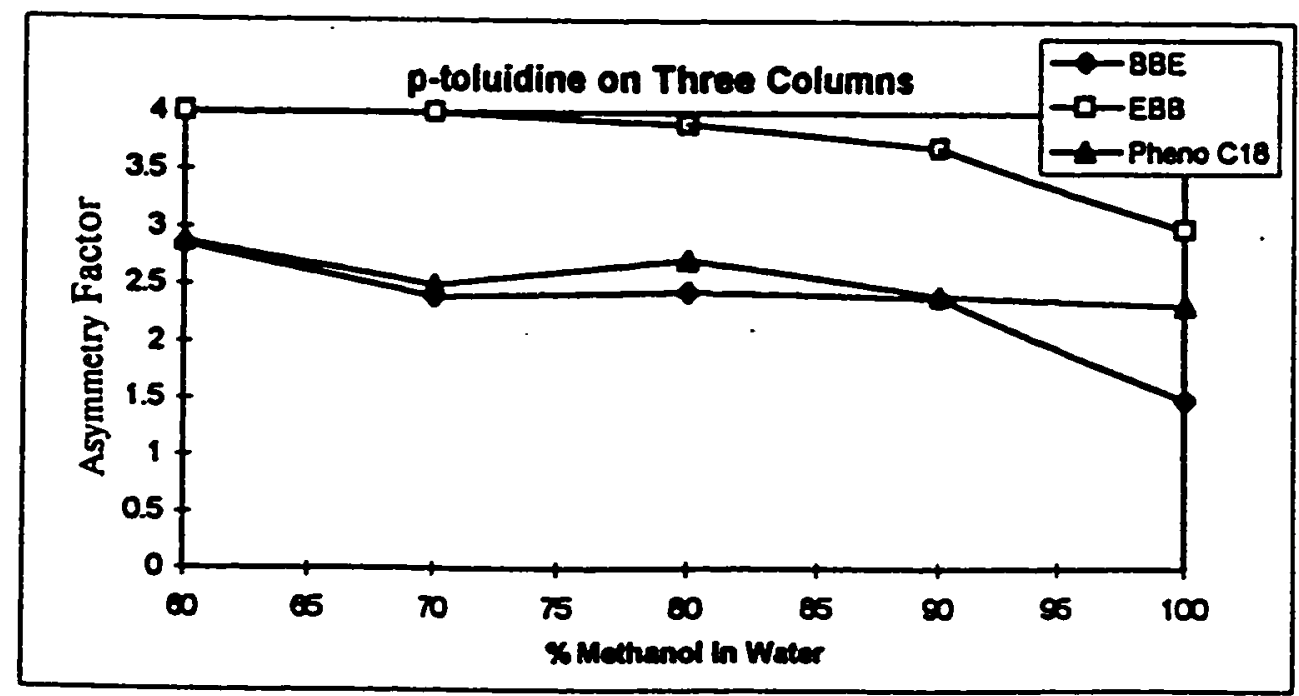

Figure 26 (a). Chromatographic studies of p-toluidine on peak symmetry. 


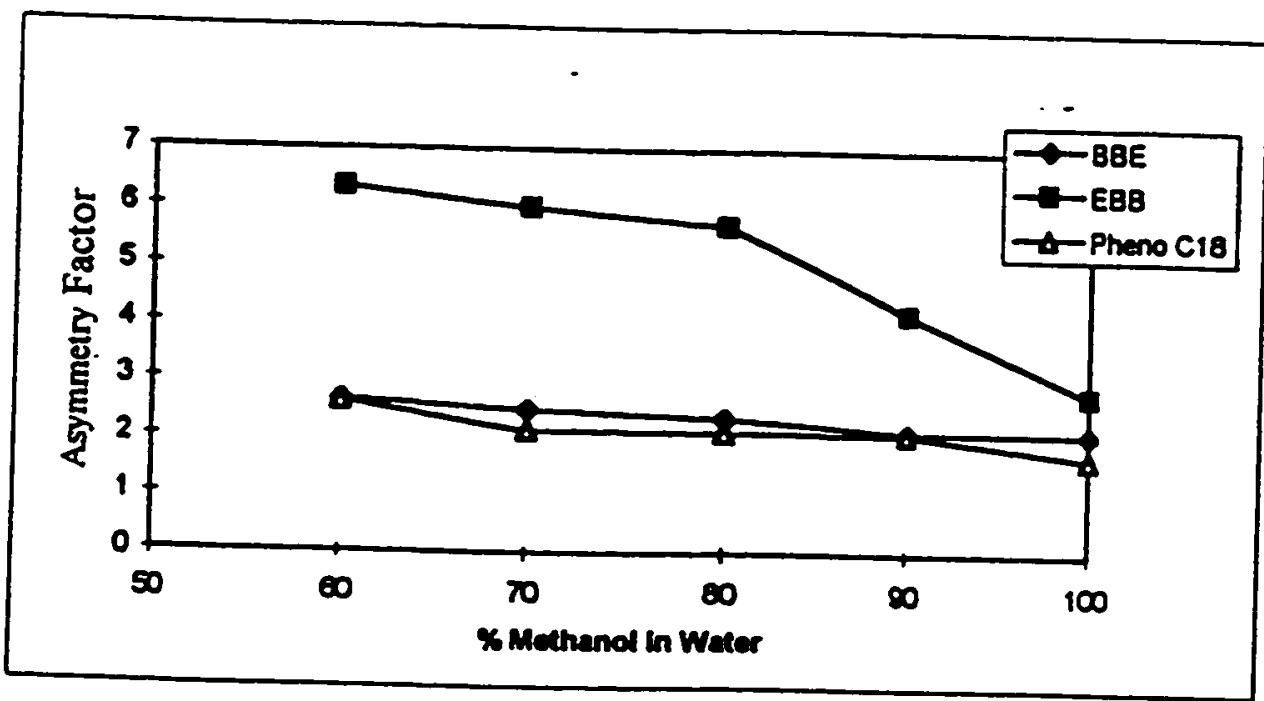

Figure 26 (b). Chromatographic studies of pyridine on peak symmetry. 


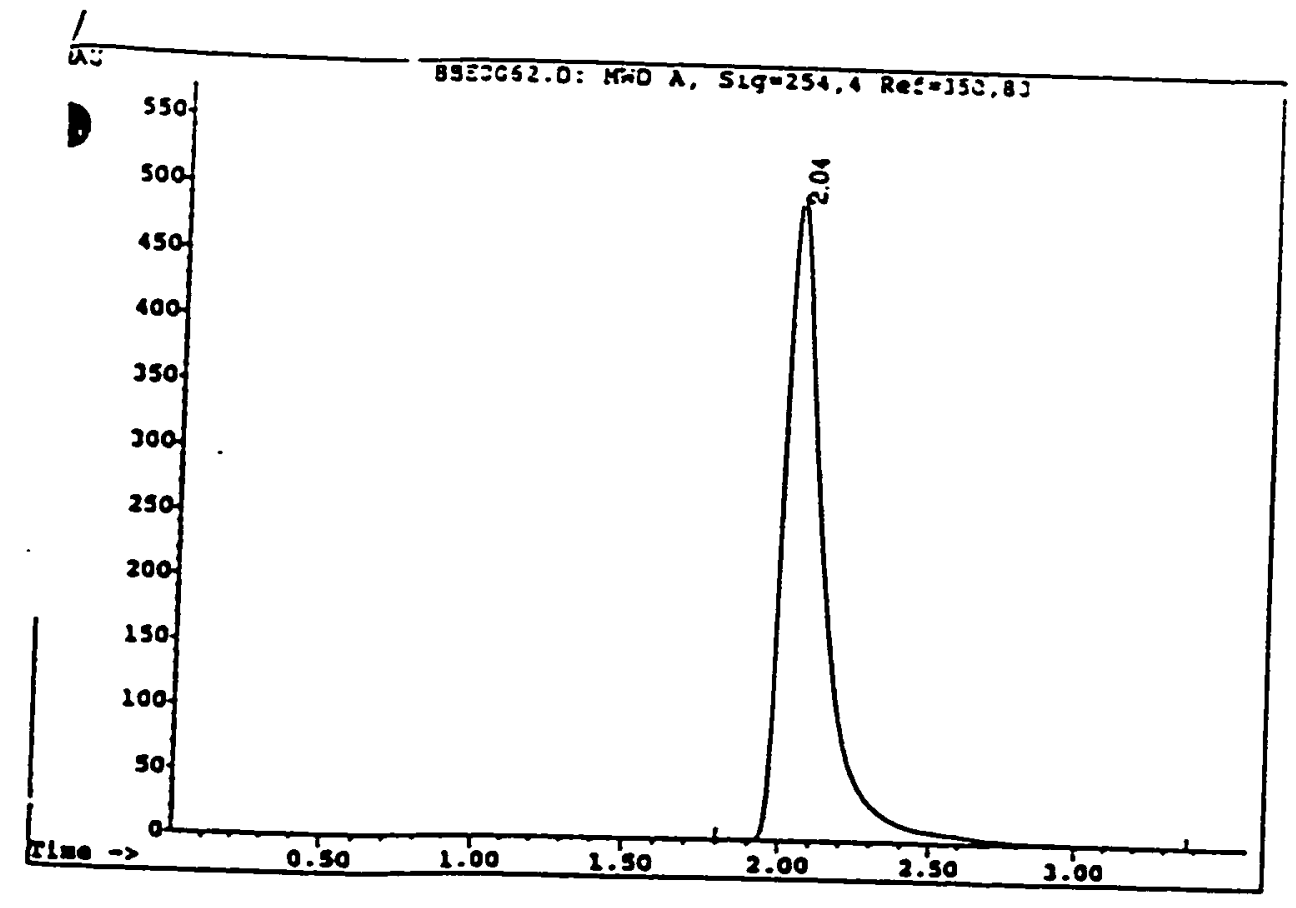

Figure 27 (a). Chromatogram of pyridine on BBE. Conditions: mobile phase: 70\% Methanol : 30\% water (v/v); Flow rate: $1 \mathrm{~mL} / \mathrm{min}$; uv detection: $254 \mathrm{~nm}$. 


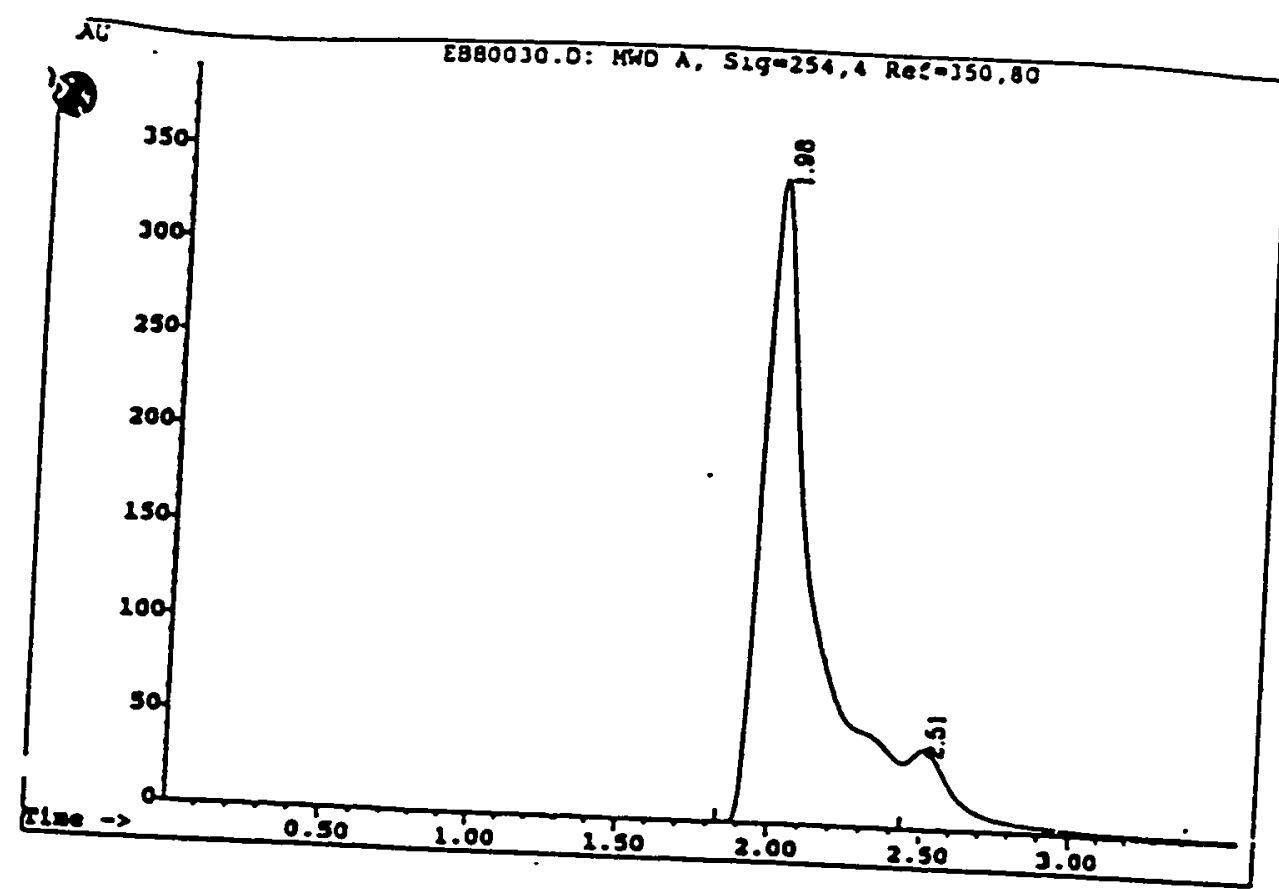

Figure 27 (b). Chromatogram of pyridine on EBB. Conditions: mobile phase: $70 \%$ Methanol : 30\% water (v/v); Flow rate: $1 \mathrm{~mL} / \mathrm{min}$; uv detection: $254 \mathrm{~nm}$. 


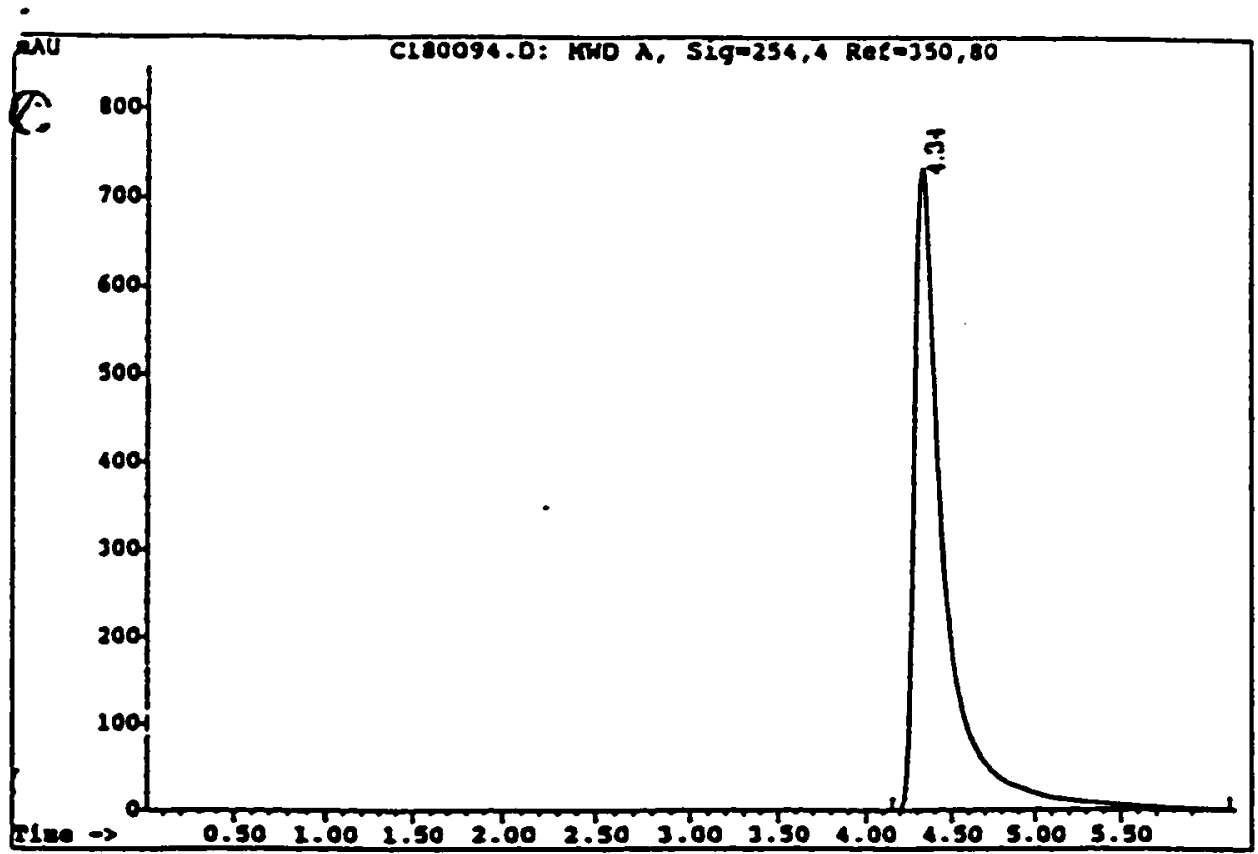

Figure 27 (c). Chromatogram of pyridine on Pheno C18. Conditions: mobile phase: 70\% Methanol : $30 \%$ water (v/v); Flow rate: $1 \mathrm{~mL} / \mathrm{min}$; uv detection: $254 \mathrm{~nm}$. 


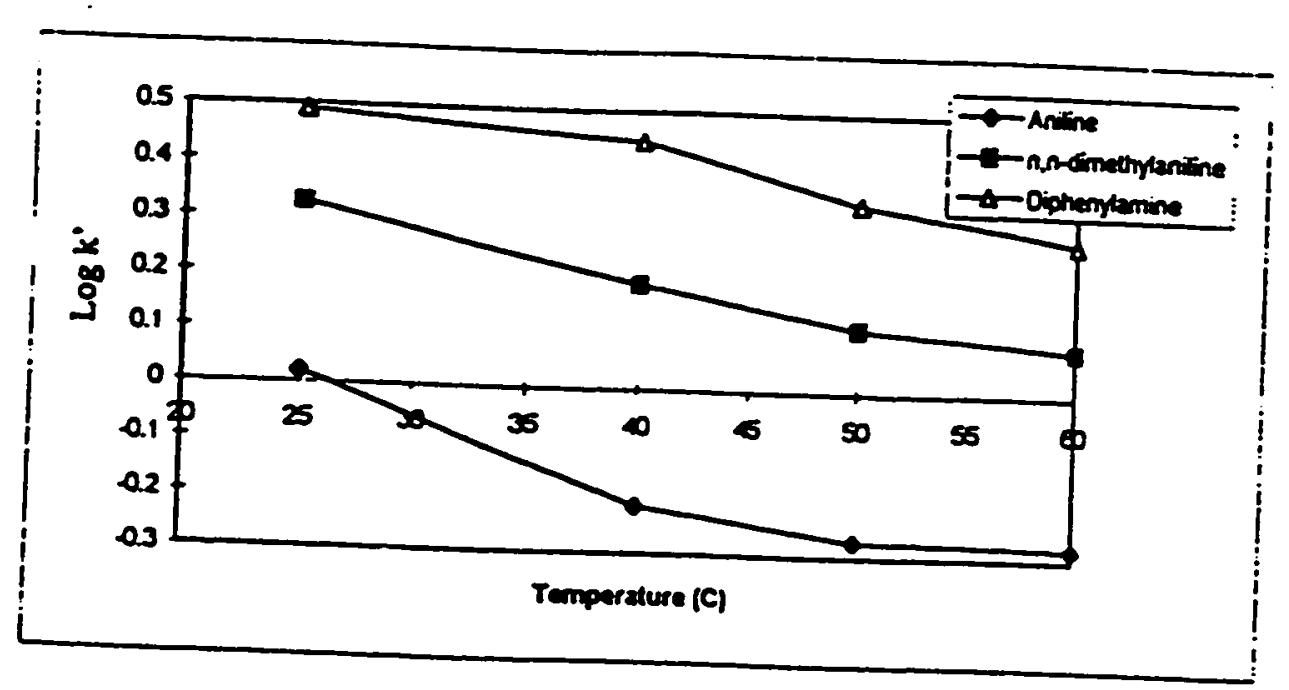

Figure 28 (a). Chromatographic study of temperature effect of retention on BBE. Conditions: mobile phase: $60 \%$ methanol : $40 \%$ water; flow rate: $1 \mathrm{~mL} / \mathrm{min}$. 


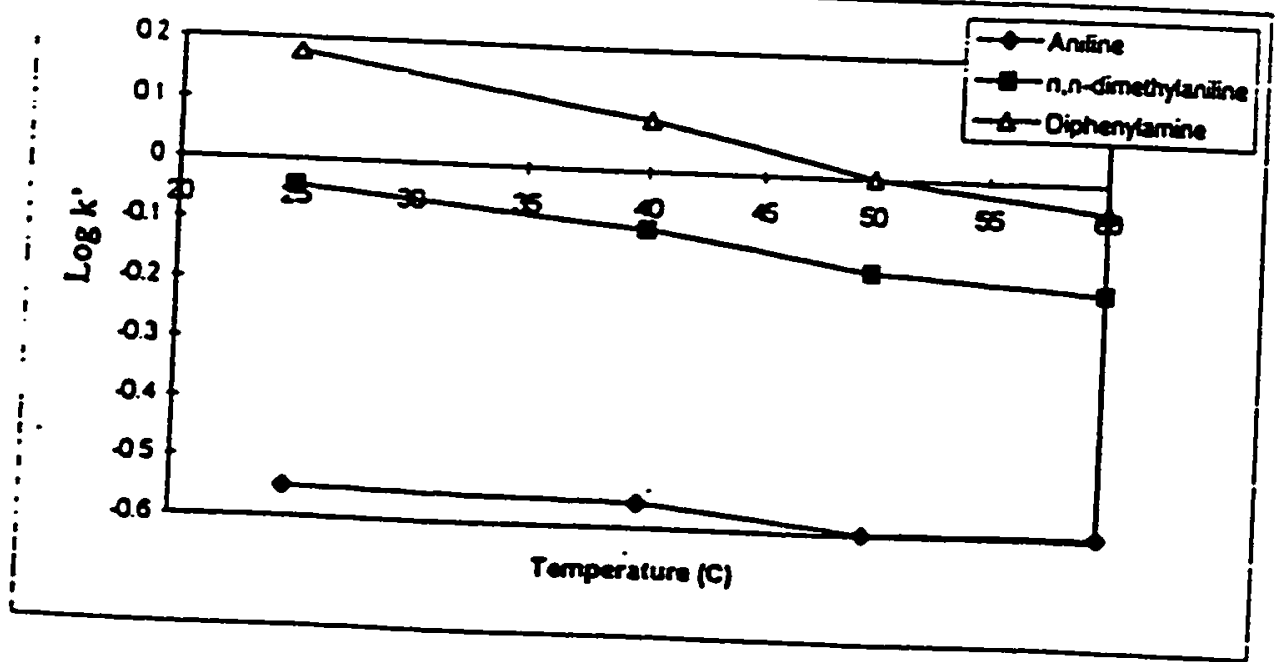
Figure 28 (b). Chromatographic study of temperature effect of retention on EBB.
Conditions: mobile phase: $60 \%$ methanol and $40 \%$ water; flow rate: $1 \mathrm{~mL} / \mathrm{min}$. 


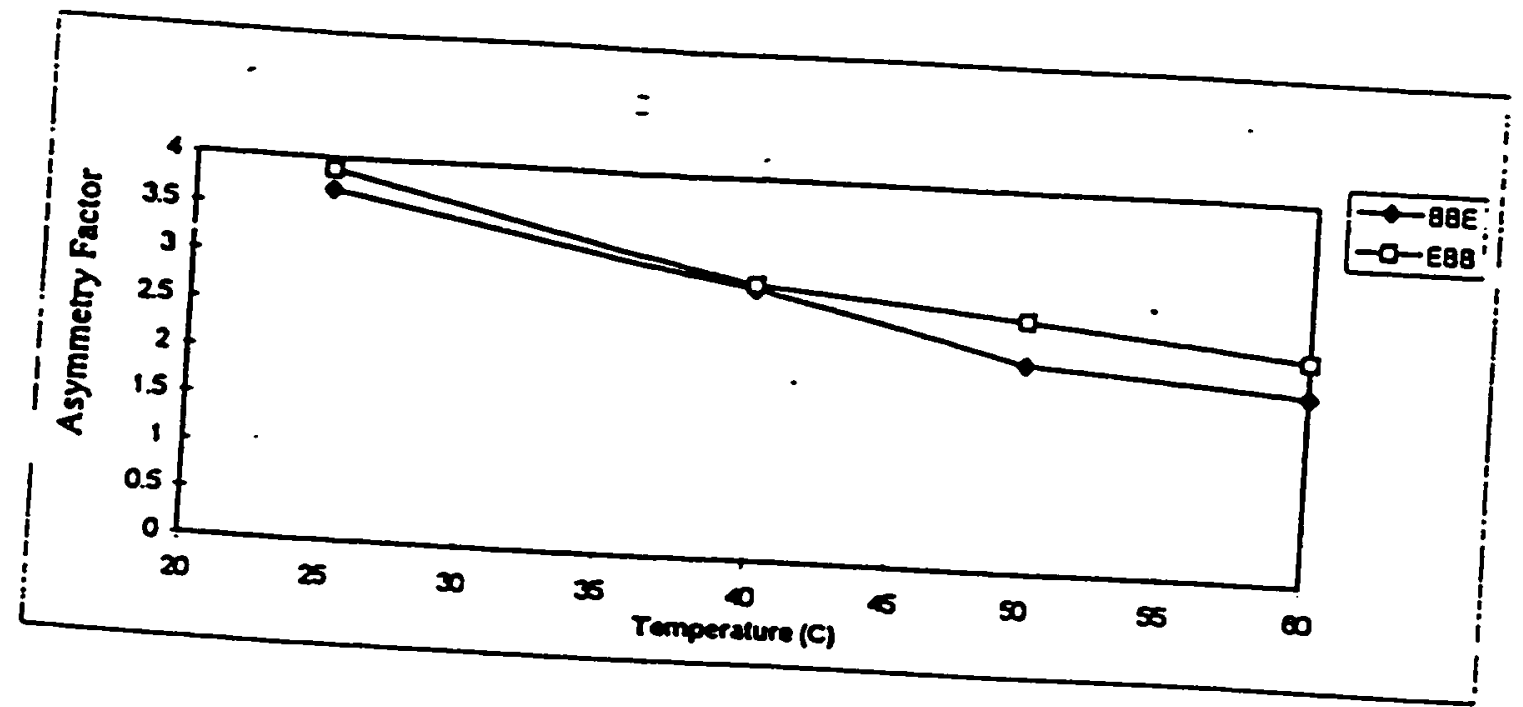

Figure 29 (a). Chromatographic study of temperature effect on peak shape. Solute: aniline. Conditions: mobile phase: $60 \%$ methanol : $40 \%$ water; flow rate: 1 mL/min. 


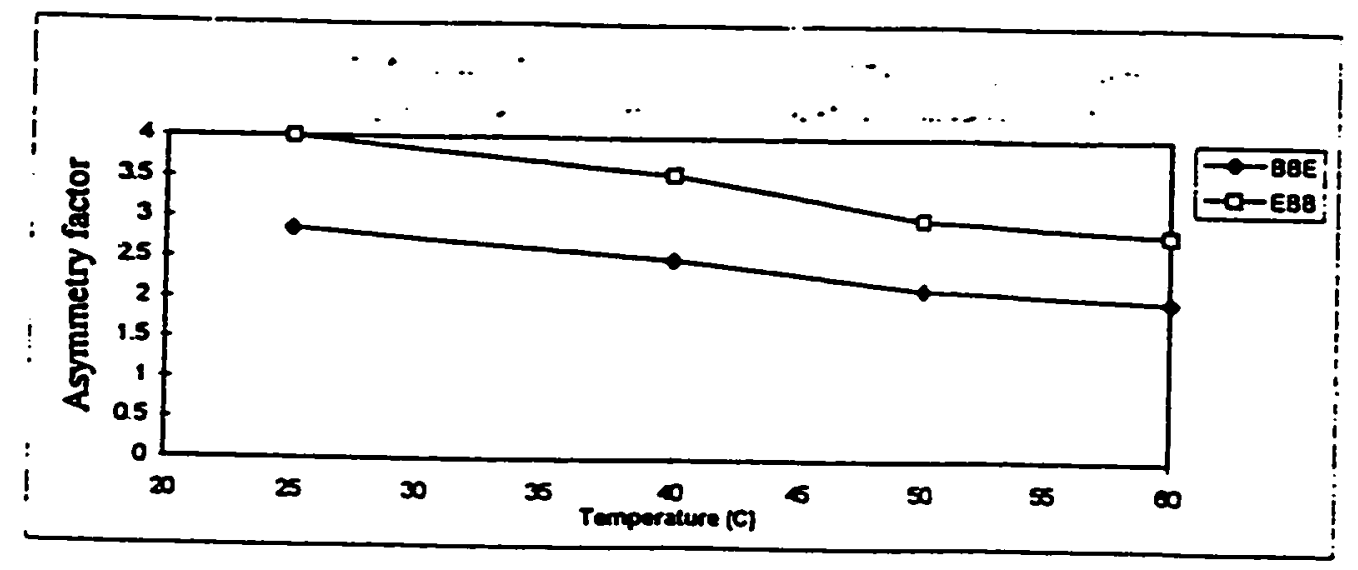

Figure 29(b). Chromatographic study of temperature effect on peak shape. Solute: n,n-dimethylaniline. Conditions: mobile phase: $60 \%$ methanol and $40 \%$ water; fow
rate: $1 \mathrm{~mL} / \mathrm{min}$. 


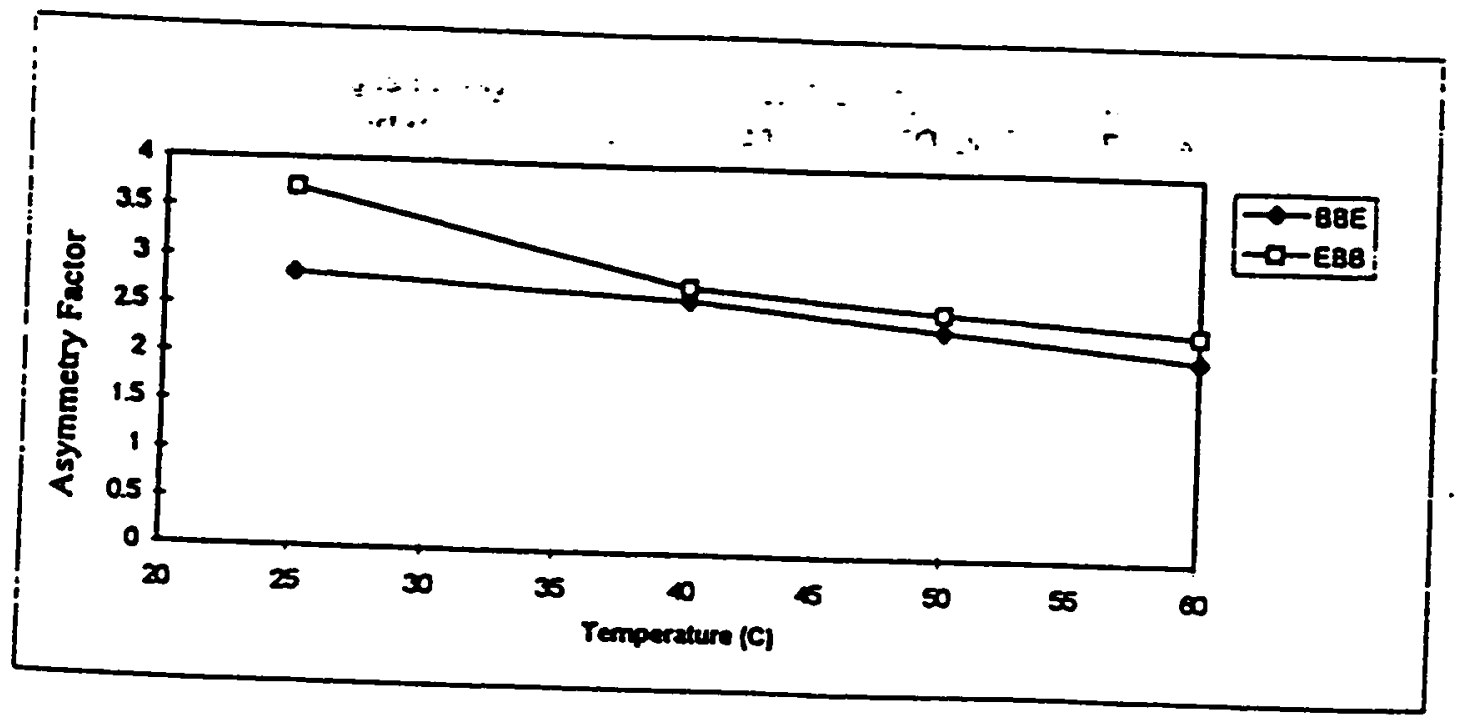

Figure 29 (c). Chromatographic study of temperature effect on peak shape. Solute: diphenylamine. Conditions: mobile phase: $60 \%$ methanol : $40 \%$ water; flow rate: 1 mL/min. 
suggested that densely bonded phases can protect silica from dissolution. In other words, endcapping molecules can help protect the silica matrix by shielding the surface, and the better endcapping is, the more protective it is.

A pH stability study was performed on BBE and EBB by passing high $\mathrm{pH}$ mobile phase through the column. It is known that as the silica matrix gradually dissolves in alkaline mobile phases, retention times will gradually decrease. Therefore, the more stable the column is, there is less retention loss. In addition, the stability of a stationary phase can be measured by the asymmetry factor $\left(\mathrm{A}_{8}\right)$. When silica deteriorates in a high $\mathrm{pH}$ environment, the asymmetry of a peak will gradually increase. Furthermore, the number of theoretical plates $(N)$ can be used to measure the stability. As more silica matrix dissolves in alkaline solution, there is poorer efficiency and thus plate number will decrease. After 4000 volumes of aggressive mobile phase at $\mathrm{pH} 9$ was passed through the BBE column, there was no obvious drop in retention. The results of changes in $k^{\prime}$ and \% change in $\mathbf{k}^{\prime}$ as mobile phase at $\mathrm{pH} 9$ is passed through the column are shown in Figure 30 and 31 . Then, 4000 volumes of mobile phase at $\mathrm{pH} 10$ was passed through the column. Second test was performed on EBB. In this case, 4000 volumes of mobile phase at $\mathrm{pH} 10$ was passed through EBB. It should be noted that EBB was not subject to 4000 volumes of mobile phase at $\mathrm{pH} 9$ prior to $\mathrm{pH} 10$. The Result is shown in Figure 32. The result shows that both $\mathrm{BBE}$ and $\mathrm{EBB}$ were quite stable under aggressive $\mathrm{pH}(10)$. However, Figure 33 shows that BBE gives lower percentage of $k$ ' change. This indicates that BBE is a more stable stationary phase than EBB. Figures 34 and 35 show the changes in asymmetry factor and the percentage of such change as high $\mathrm{pH}$ mobile phase is passed 


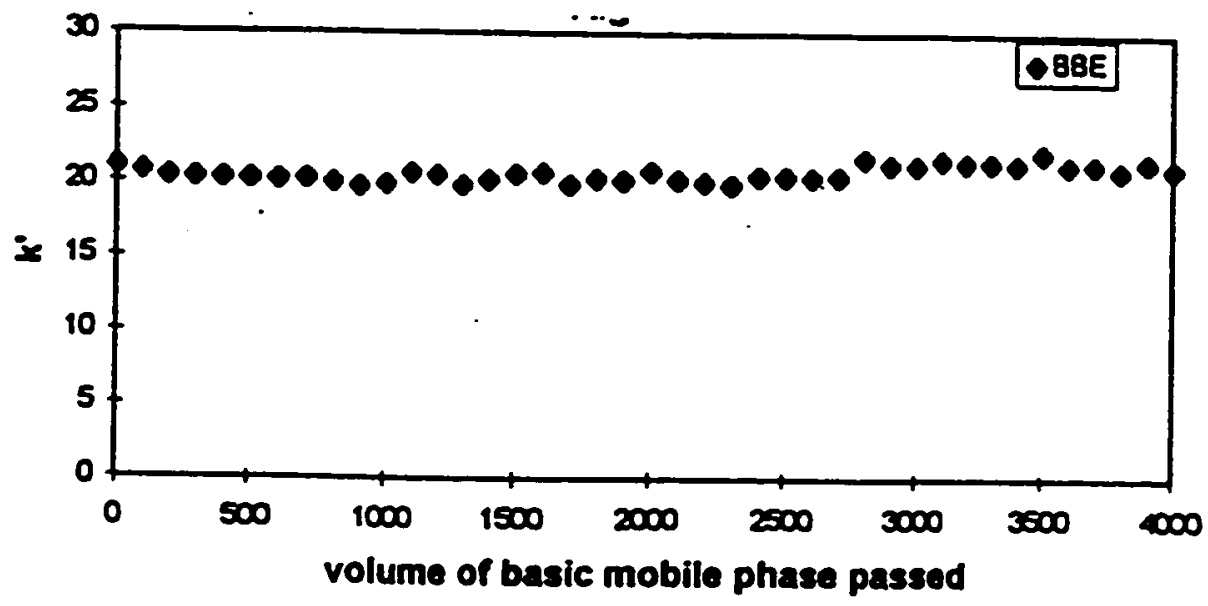

Figure 30. High pH stability chromatographic study on retention. Analyte: pyrene. Conditions: mobile phase: $60 \%$ methanol : $40 \%$ water containing $0.1 \mathrm{M} \mathrm{N} 2 \mathrm{OH}$ at pH 9; llow rate: $1 \mathrm{~mL} / \mathrm{min}$. 


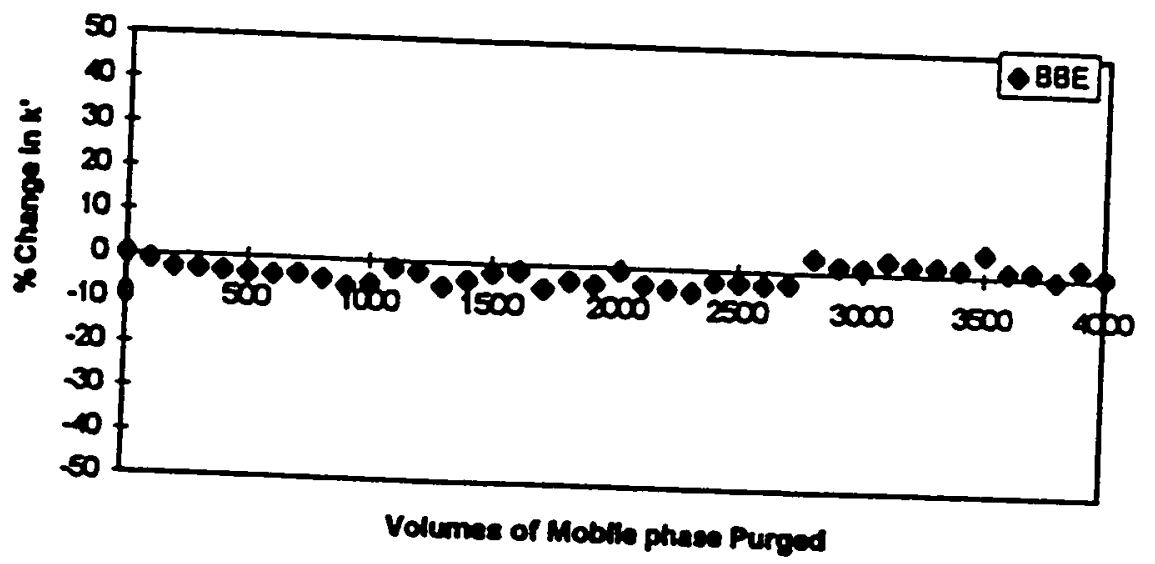

Figure 31. High pH stability chromatographic study on \% change in k'. Analyte: pyrene. Conditions: mobile phase: $60 \%$ methanol : $40 \%$ water containing $0.1 \mathrm{M}$ NaOH at pH 9; How rate: 1 mL/min. 


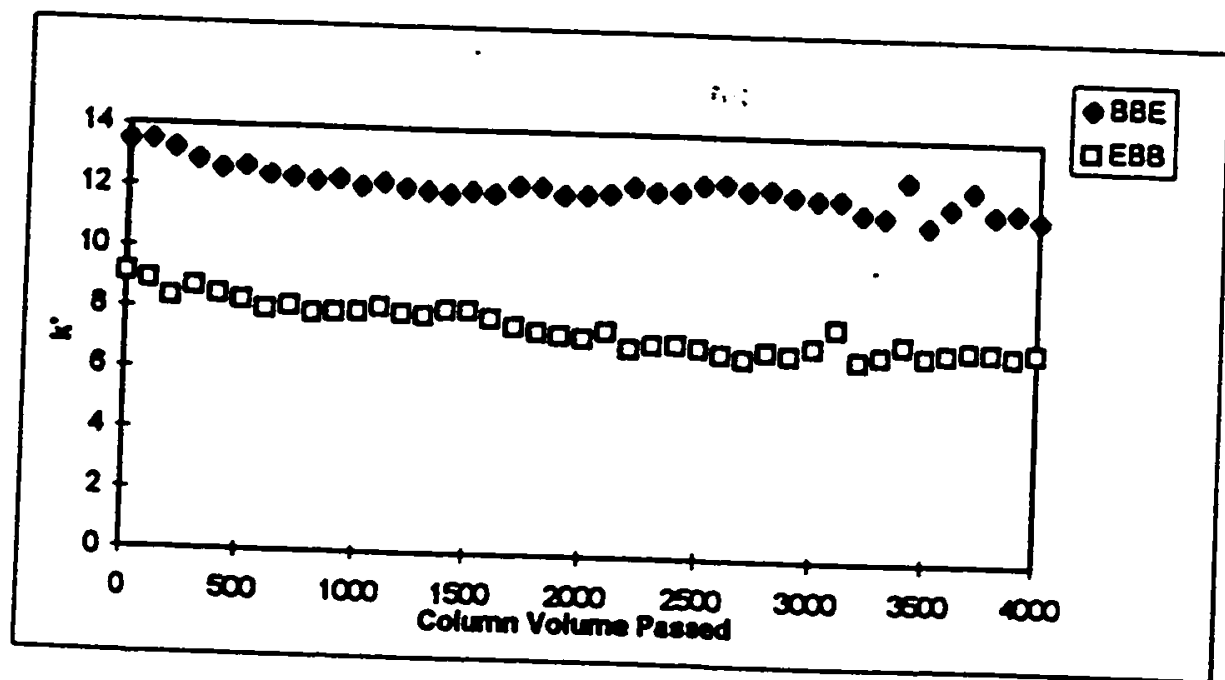

Figure 32. High pH stability chromatographic study on retention. Analyte: pyrene. Conditions: mobile phase: $60 \%$ methanol : $40 \%$ water containing $0.1 \mathrm{M} \mathrm{NaOB}$ at
pR 10; flow rate: $1 \mathrm{~mL} / \mathrm{min}$. 


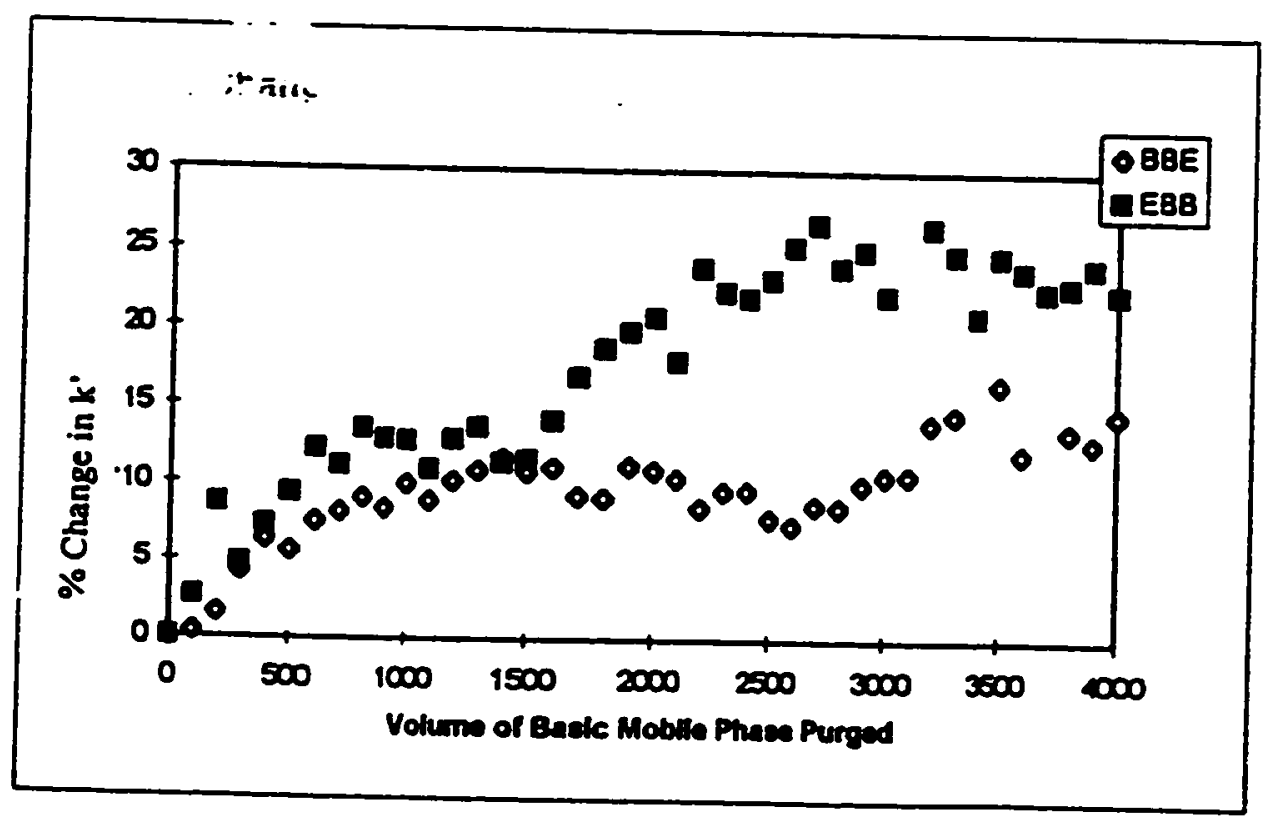

Figure 33. Aigh pE stability chromatographic study on \% change in k'. Analyte: pyrene. Conditions: mobile phase: $60 \%$ methanol : $40 \%$ water containing $0.1 \mathrm{M}$ NaOE at pR 10; flow rate: $1 \mathrm{mi} / \mathrm{min}$. 


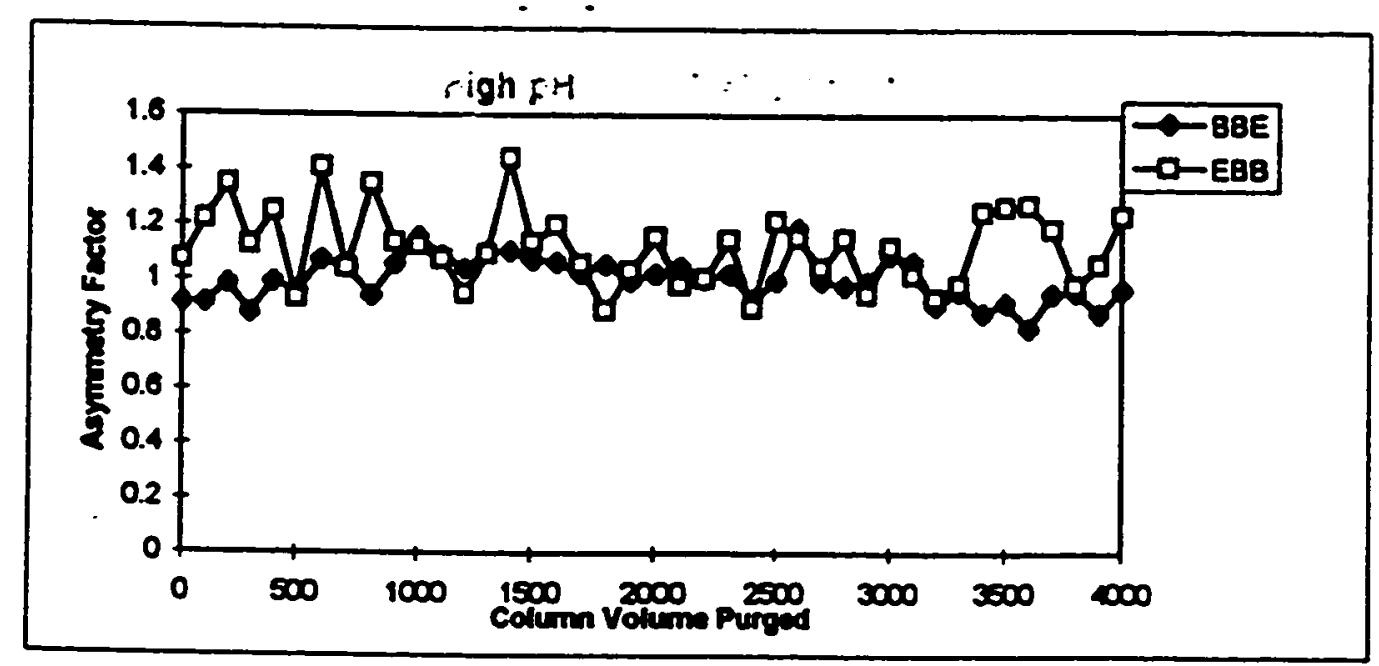

Figure 34. High pH chromatographic stability study on peak shape. Analyte: pyrene. Conditions: mobile phase: $60 \%$ methanol : $40 \%$ water containing $0.1 \mathrm{M}$ NaOH at pH 10; flow rate: $1 \mathrm{~mL} / \mathrm{min}$. 


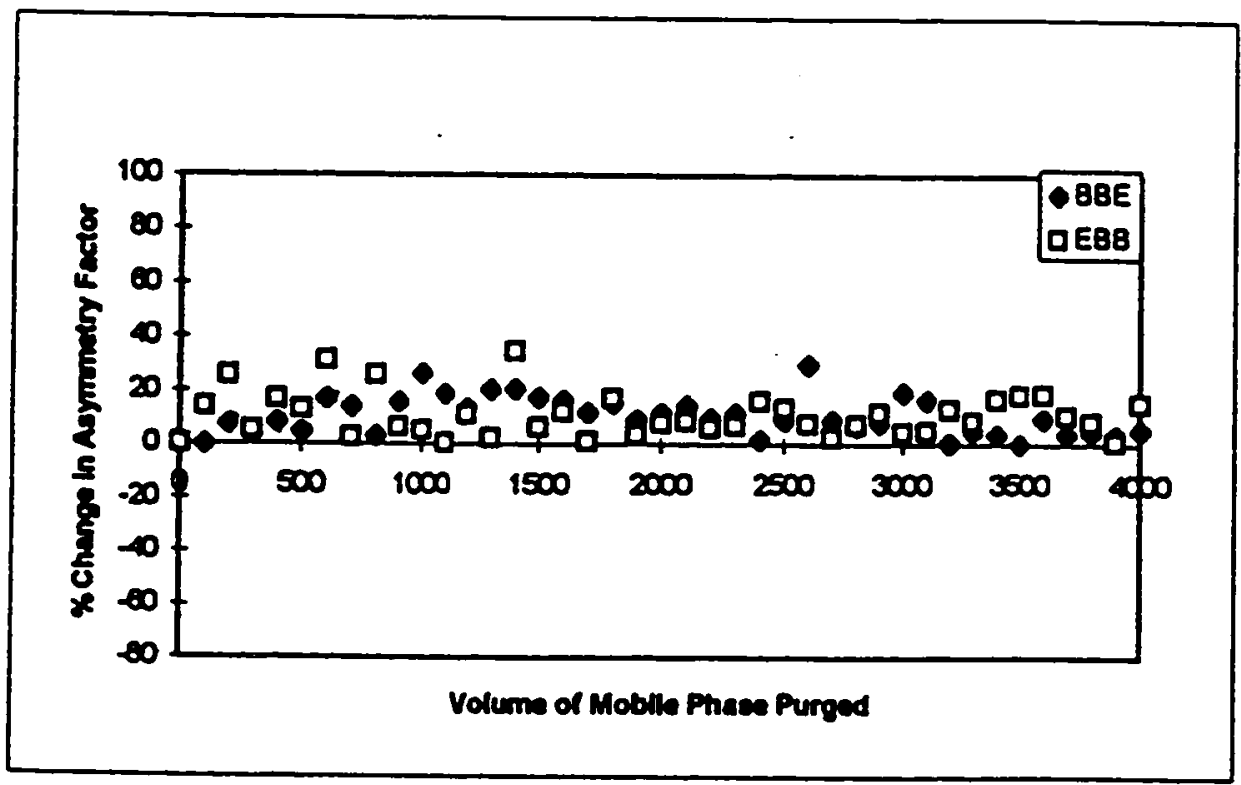

Figure 35. High pH chromatographic stability study on \% change in asymmetry factor. Analyte: pyrene. Conditions: mobile phase: $60 \%$ methanol : $40 \%$ water containing $0.1 \mathrm{M} \mathrm{NaOH}$ at $\mathrm{pH} \mathrm{10}$; flow rate: $1 \mathrm{~mL} / \mathrm{min}$. 


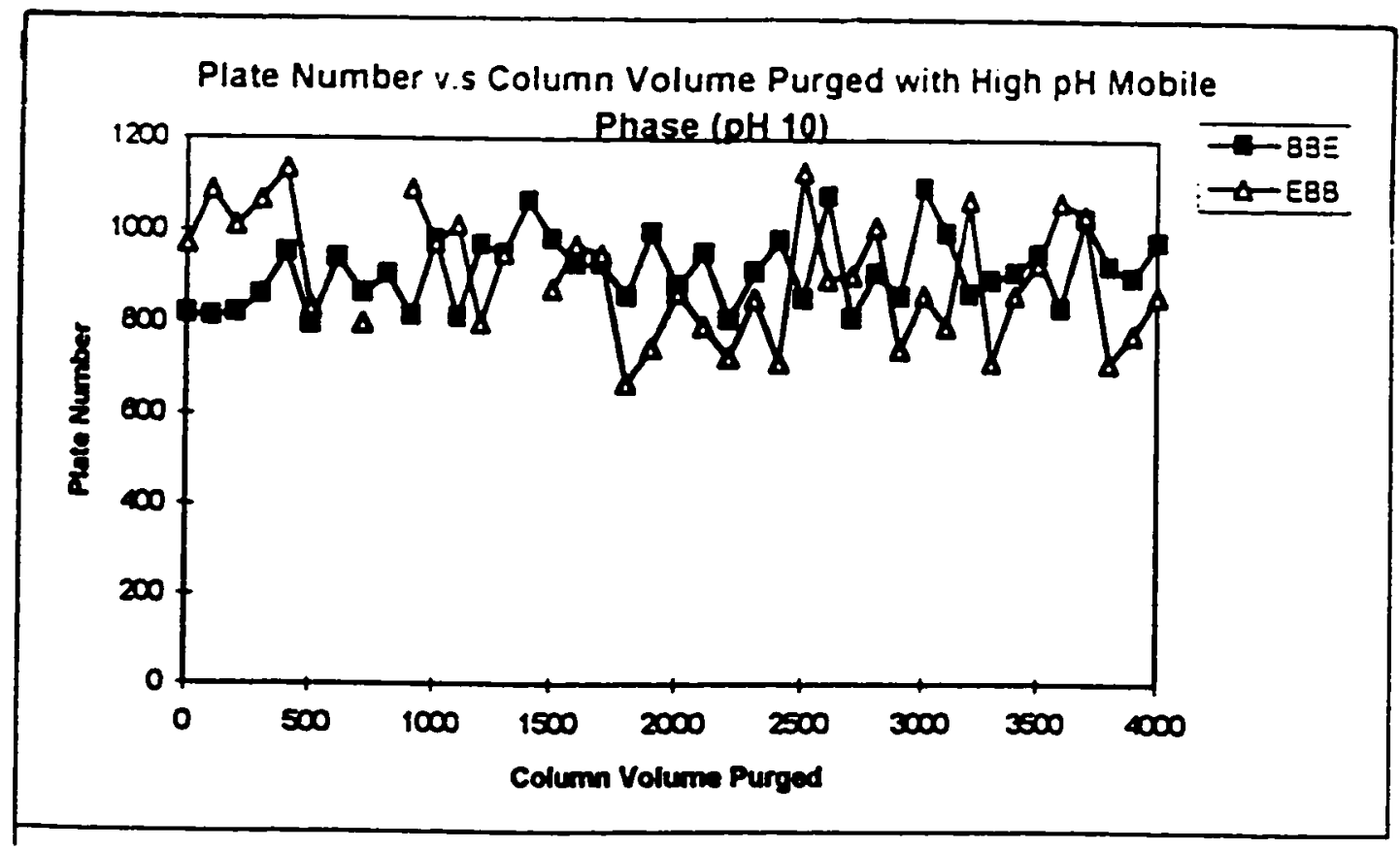

Figure 36. High pH chromatographic stability study on theoratical plates. Column: BBE. Analyte: pyrene. Conditions: mobile phase: $60 \%$ methanol : $40 \%$ water containing 0.1 M NaOB at pH 10; fow rate: $1 \mathrm{~mL} / \mathrm{min}$. 


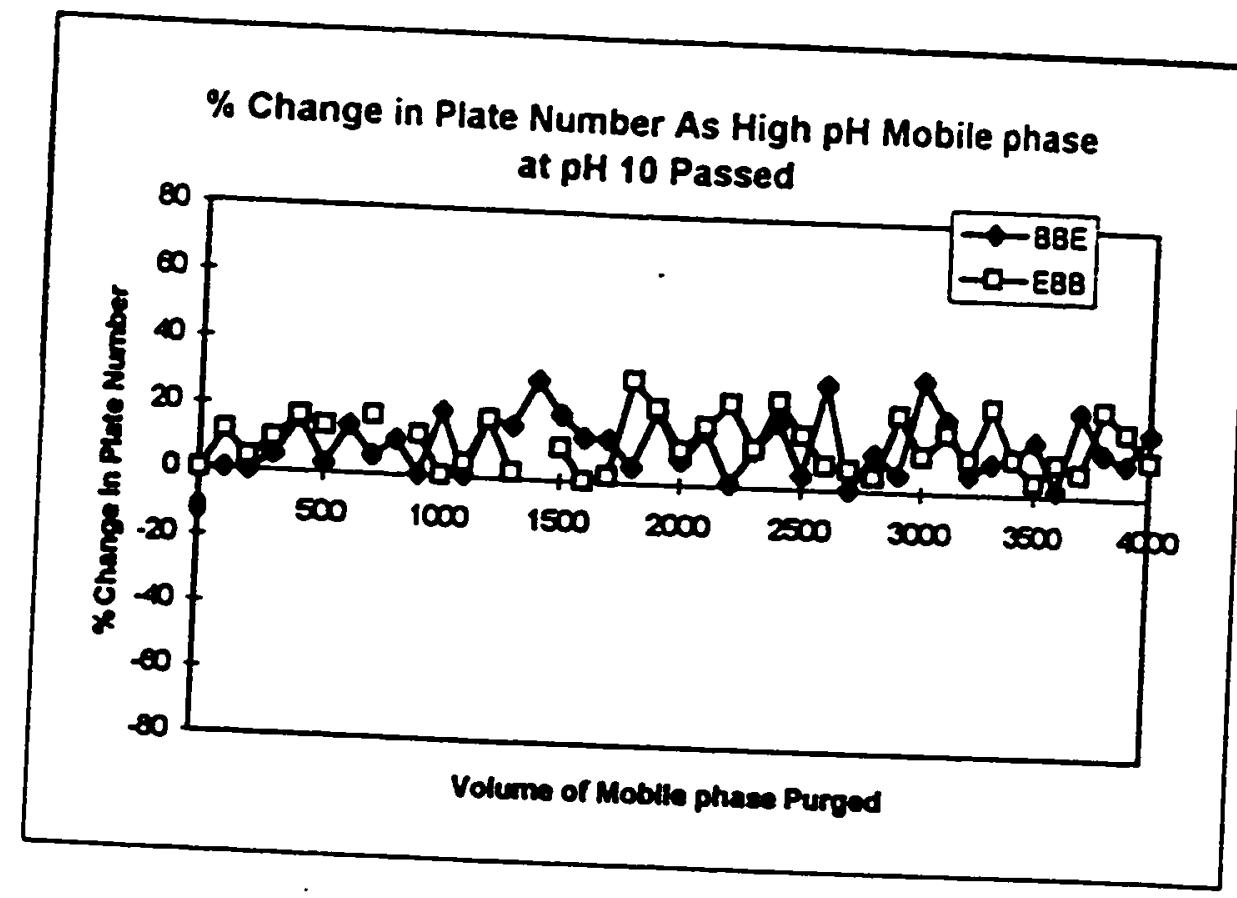

Figure 37. High pH chromatographic stability study on \% change in theoratical plate. Column: BBE. Analyte: pyrene. Conditions: mobile phase: $60 \%$ methanol : $40 \%$ water containing $0.1 \mathrm{M} \mathrm{NaOH}$ at $\mathrm{pH} 10$; fow rate: $1 \mathrm{~mL} / \mathrm{min}$. 
through the columns. The result shows that BBE gives more stable and better peak shapes. This indicates that BBE surface is better protected. Figures 36 and 37 show the changes in plate numbers and percentage of such change as aggressive mobile phase is passed through the columns. The result also indicates that BBE is slightly better than EEB in column efficiency.

\section{Low pH Stability Study}

Silica is stable in acidic solution, but bonded phases made by organosilane undergo hydrolysis at siloxane linkage. ${ }^{16}$ As a result, the bonded phase becomes lost, and the surface is converted back to silanol. It is suggested that side chains can protect the silica surface, and thus provide higher stability. Therefore, the endcapping can help protect the silica surface from hydrolytical attack by protons. ${ }^{16}$ A low $\mathrm{pH}$ stability test is conducted by passing 4000 volumes of mobile phase containing $2 \%$ TFA pH 2 through the BBE column. A decrease in retention is observed since the bonded phase will gradually be lost under acidic conditions. The results are shown in Figures 38 and 39. The result shows that there is no obvious drop in retention as 4000 volumes of mobile phase at $\mathrm{pH} 2$ passed through the column. This indicates the BBE column is very stable under low pH conditions. Therefore, the endcapping was very successful. 


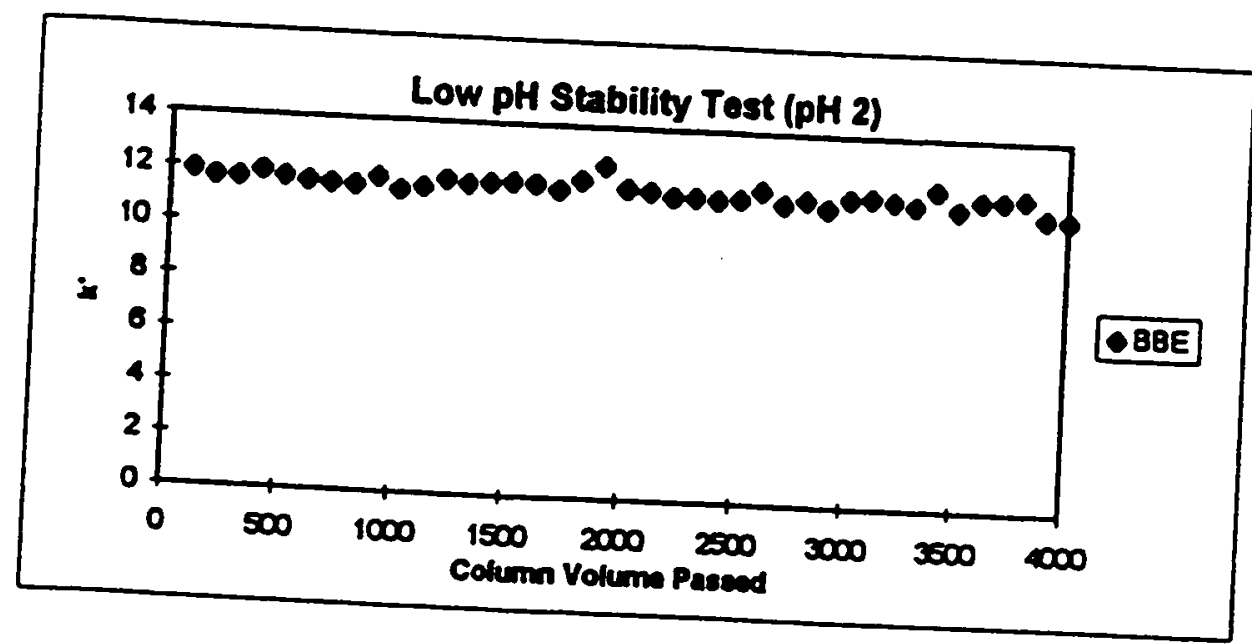

Figure 38. Low pH stability study on BBE. Analyte: pyrene. Conditions: mobile phase: $60 \%$ methanol : $40 \%$ water containing $2 \%$ TFA at pH 2; flow rate: 1
mL/min. 


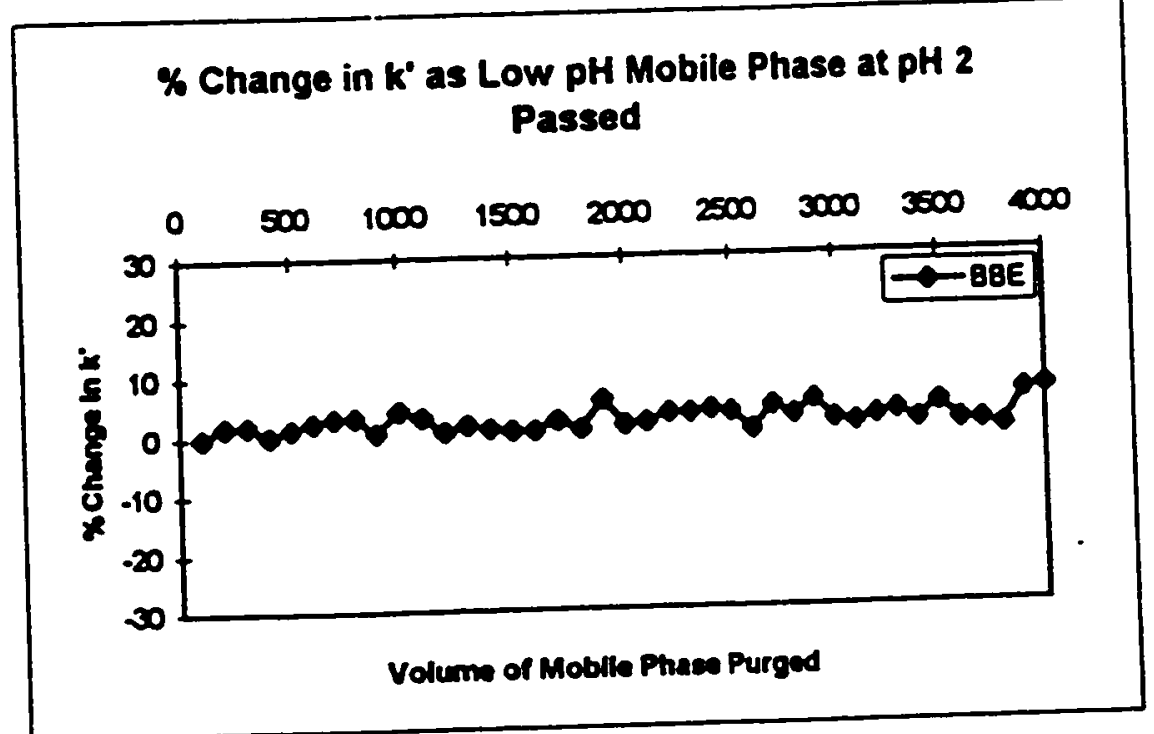

Figure 39. Low pH stability study of \% change in k' on BBE. Analyte: pyrene. Conditions: mobile phase: $60 \%$ methanol and $40 \%$ water containing $2 \%$ TFA at pH 2; Ilow rate: $1 \mathrm{~mL} / \mathrm{min}$. 


\section{CHAPTER IV}

\section{CONCLUSIONS}

Spectroscopic characterization, including DRIFT and CP-MAS NMR, show that trimethylsilyl groups have been bonded to the surfaces for both before and after C-18 bonding. The final products of BBE and EBB look different spectroscopically. Also. elemental chemical analysis showed that EBB has lower percent carbon loading and lower surface coverage. This is probably due the fact that in EBB, trimethylsilyl groups from endcapping have potentially blocked access of $\mathrm{C}-18$ to hydrides on the surface. On the other hand, small trimethylsilyl groups could easily squeeze into the C-18 pockets on the surface, and react with some of the residual silanols. As a result, a more hydrophobic hydrocarbon phase has been loaded on the BBE surface. This is confirmed by chromatographic data in which BBE gives better separation for PE-UTM and SRM 869 mixtures. Chromatographic studies of basic compounds show that BBE gave better peak shapes than EBB. This indicates that endcapping by BBE is a better method than that of EBB in order to remove residual silanols. BBE even gave better results than Pheno $\mathrm{Cl} 8$ in basic compound analysis. This further proved the success of endcapping by BBE. The temperature study indicates that indeed increasing temperature does improve peak shape and results in less tailing. Finally, pH stability studies indicates that both BBE and EBB were very stable at both low and high $\mathrm{pH}$. 


\section{CHAPTER V}

\section{REFERENCES}

1. Lindsay, Sandie; High Performance Liquid Chromatography, John Wiley \& Sons, 1993, Chapter 1 and Chapter 2.

2. Dorsey, John G.; Cooper, William T.; Anal. Chem. 1994, 66, 857A-867A.

3. Majors, Ronald E.; LC-GC 1997, May, S8-S19.

4. Pesek, J. J., Matyska, M. T.; Sandoval, J. E.; Williamsen, E. J; J. Liq. Chrom. \& Rel. Technol. 1996, 19 (17\&18), 2843-2865.

5. Sandoval, J. E.; Pesek, J. J.; Auvinen, Mirva; Jonsson, Elisabet; Chu Chao-Hau; Anal. Chem. 1993, 65, 808-816.

6. Pesek, J. J.; Williamsen, Eric J.; J. Chromatogr, Lib., 1995, 57, 371-401.

7. Sander, L.; Wise, S.; CRC critical Reviews in Analytical Chemistry, 1987, 18(4), 319.

8. Nawrocki, Jacek; J. of Chromatogr. A, 1997, 779, $29-71$.

9. Marshail, David B.; Cole, C.L.; Norman, Arland D.; J. of Chromatographic Science, 1987, 25, 262-266.

10. Marshall, David B.; Lochmuller, C.H.; Analytica Chimica Acta, 1982, 142, 63-72.

11. Pavia, Donald. L.; Lampman, Gary M.; Kriz, George S.; Introduction to Spectroscopy: A guide for students of Organic Chemistry, Saunders Golden Sunburst Series, 1979, Chapter 2 and Chapter 3.

12. Skoog, Douglas; West, Donald; Principles of Instrumental Analysis, Saunders Golden Sunburst Series, 1980, 8 and Chapter 14.

13. Pesek, J. J.; Sandoval, J. E.; Anal. Chem., 1991, 63, 2634-2641.

14. Akapo, S. O.; Dimandja, J. D.; Pesek, J. J.; Matyska, M. T.; Anal. Chem., 1996, 68, 1954-1959.

15. Synder, L. R.; Dolan, J. W,; Molnar, I.; Djordjevic, N. M.; LC-GC, 1997, 15(2), 136151. 
16. Ludwig, Richard C.; Reid, Terrence S.; Henry, Richard A.; Duff, Keith J.; LC-GC, 1998, 16(2), 158-168.

17. Kirland, J. J.; van Straten, M. A.; Claessens, H.A.; J. of Chromatogr. A., 1995, 691, 3-19.

18. Kirland, J. J.; J. of Chromatographic Science, 1996, 34, 309-312. 

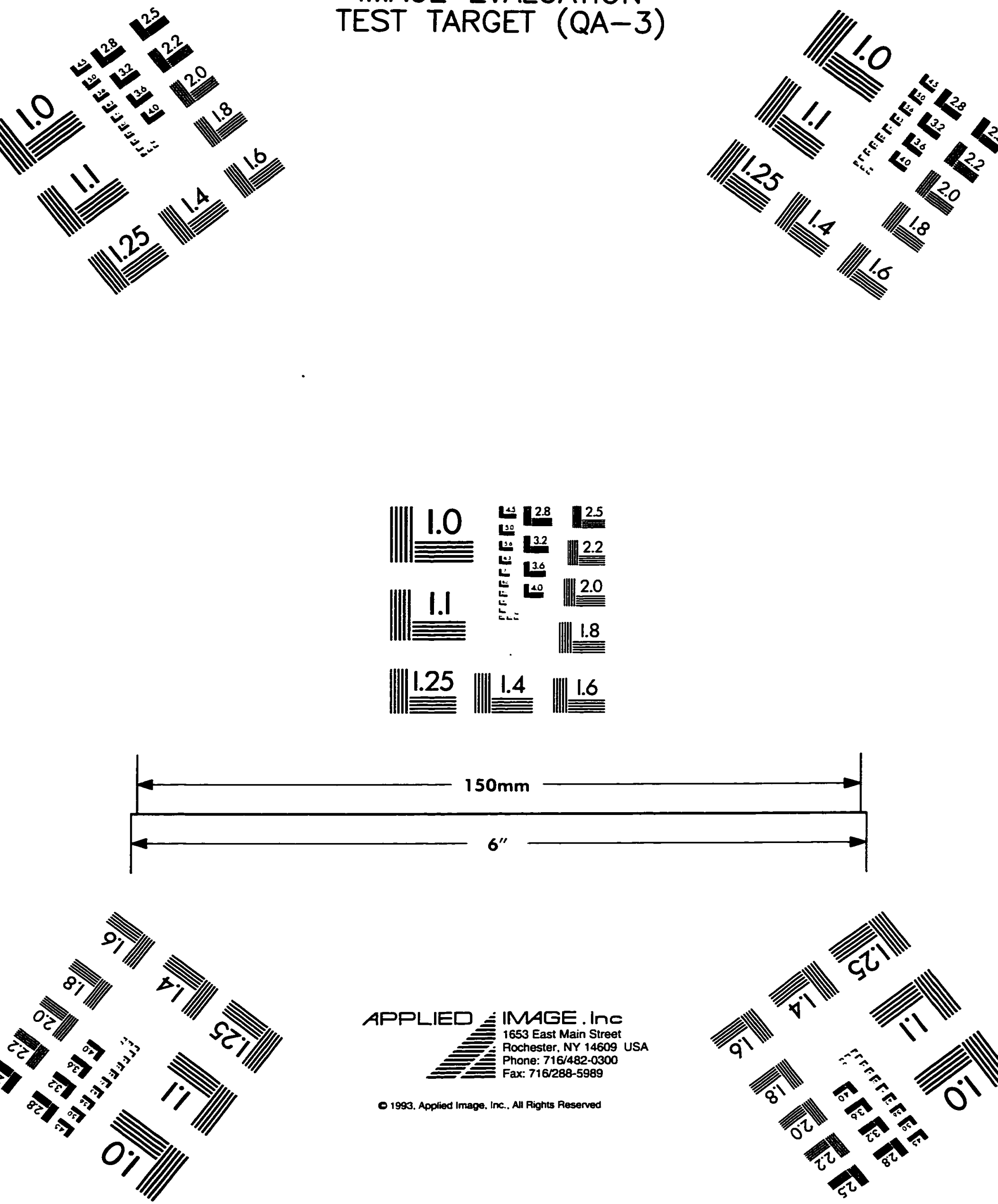